\title{
Autonomous Infrastructure Based Multihop Cellular
}

NETWORKS

by

Mark DeFaria

A thesis submitted in conformity with the requirements

for the degree of Doctor of Philosophy

Graduate Department of Electrical and Computer Engineering University of Toronto

Copyright (c) 2010 by Mark DeFaria 


\begin{abstract}
Autonomous Infrastructure Based Multihop Cellular Networks

\author{
Mark DeFaria \\ Doctor of Philosophy \\ Graduate Department of Electrical and Computer Engineering \\ University of Toronto
}

In a multihop cellular network, mobile terminals have the capability to transmit directly to other mobile terminals enabling them to use other terminals as relays to forward traffic towards the base station. Previous studies of networks consisting of a single cell found that the SINR in a multihop cellular network is slightly lower than in a traditional cellular network. However, multihop cellular networks may have a higher capacity than traditional cellular networks due to their potential for lower intercell interference. For this reason, the effects of intercell interference are investigated in this thesis. Our simulations of a network with many cells show that multihop cellular networks have a higher SINR than traditional cellular networks due to the near elimination of intercell interference.

However, multihop cellular networks still suffer from large amounts of interference surrounding the base station because all traffic either emanates or is destined to the base station making it the capacity bottleneck. To resolve this problem, we propose a novel architecture called the autonomous infrastructure multihop cellular network where users can connect their mobile terminals to the backbone network giving them the functionality of an access point. Access points receive traffic from other terminals and send it directly onto the backbone, as would a base station. This reduces the traffic handled by the base station and increases network capacity. Our analysis and simulations show that in autonomous infrastructure multihop cellular networks, the SINR at the base station 
is higher, the power consumption is lower and the coverage is better than in normal multihop cellular networks.

Access points require parameters like their transmission range to be adjusted autonomously to optimal levels. In this thesis, we propose an autonomous pilot power protocol. Our results show that by adjusting a parameter within the protocol, a required coverage level of terminals can be specified and achieved without knowledge of the location or density of mobile terminals. Furthermore, we show that the protocol determines the transmission range that is optimal in terms of SINR and power consumption that achieves the required coverage while effectively eliminating the bottleneck that existed at the base station. 


\section{Acknowledgements}

First and foremost, I would like to express my gratitude to my supervisor Prof. Elvino Sousa for providing me with the opportunity to research with him. His invaluable support, guidance and encouragement have provided me with the motivation to constantly excel. I truly believe that his deep understanding, vision and insight have greatly contributed to the completion of this thesis. I will always be grateful for his guidance not only on academic research but also on trends in the industry, career choices and teaching.

I would like to thank Prof. Ben Liang and Prof. Teng Joon Lim for being on my Ph.D. thesis supervisory and examination committees and for providing me with their invaluable comments. Their feedback has greatly improved the quality of this thesis. I would also like to thank other members of the examination committee, Prof. Mireille Broucke, Prof. Frank Kschischang and the external examiner Prof. Roshdy Hafez.

I would also like to acknowledge members of the Wireless Communications and Systems Laboratory, past and present, with whom I have worked with over the years. I would like to thank Yongfeng Chen, Roya Doostnejad, Amir Ghasemi, Bijan Golkar, Mahdi Lotfinezhad, Yangyang Li, Ivo Maljevic and Leon Yen. I would also like to commend the administrative assistants of the Communications Group, Diane Silva and Mary Stathopoulos for the excellent work that they do at the university on a daily basis.

I would also like to address a special thanks to my parents for their love and support throughout my journey as a graduate student. I greatly appreciate the sacrifices that they have made so that I could achieve my goals. I would also like to thank Wendy Monosky who has always been highly supportive and has provided me with a great source of inspiration and love. I would also like to thank the rest of my family, especially my grandmother who always believed in and encouraged our pursuit of higher education. I am very glad that she will, at the age of 96 , have the opportunity to see me graduate.

Last but not least, I would like to thank the University of Toronto, Rogers Communications and Telus for generously supporting this research. 


\section{Contents}

Abstract

Acknowledgements $\quad$ iv

List of Figures $\quad x$

1 Introduction 1

1.1 Motivation . . . . . . . . . . . . . . . . . 2

1.2 Problem Description . . . . . . . . . . . . . . . . . . . 4

1.3 Thesis Contributions . . . . . . . . . . . . . . . . . . 8

1.4 Organization of Thesis . . . . . . . . . . . . . . . . . 12

2 Background and Related Literature $\quad 13$

2.1 Traditional Cellular Networks . . . . . . . . . . . . . . . . . . . 13

2.1.1 Cellular Structure . . . . . . . . . . . . . . . . . . . 14

2.1.2 Evolution of Traditional Cellular Networks . . . . . . . . . . . . . 14

2.1.3 CDMA Cellular Networks . . . . . . . . . . . . . . . . 17

Spread Spectrum . . . . . . . . . . . . . . . . . . 18

Spreading Codes . . . . . . . . . . . . . . . . . 21

Power Control . . . . . . . . . . . . . . . . . . . 22

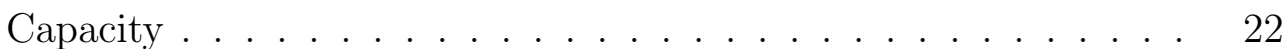

2.2 Ad Hoc Networks . . . . . . . . . . . . . . . . . . . . . . . . . . 24 
2.2 .1 Limitations . . . . . . . . . . . . . . . . 25

2.3 Multihop Cellular Networks _. . . . . . . . . . . . . . . . . . 26

2.3.1 Network Architecture . . . . . . . . . . . . . . . . 26

2.3 .2 Limitations . . . . . . . . . . . . . . . . . 27

2.3.3 Variations of Multihop Cellular Networks . . . . . . . . . . . . . 29

2.3.4 Previous Works . . . . . . . . . . . . . . . . . . . 32

3 Multihop Cellular Network System Model 36

3.1 Network Model . . . . . . . . . . . . . . . . . . . 37

3.2 Transmission Model . . . . . . . . . . . . . . . . . . . . . . . 39

3.3 Spreading Codes . . . . . . . . . . . . . . . . . . 40

3.4 Communication Channels . . . . . . . . . . . . . . . . . . . 41

3.5 Routing Algorithm . . . . . . . . . . . . . . . . . . . . . 41

3.6 Time Division Duplex CDMA . . . . . . . . . . . . . . . . . 45

3.7 Power Control . . . . . . . . . . . . . . . . . . . . . . . . . 46

3.8 Simulation Parameters . . . . . . . . . . . . . . . . . . . . . . 48

4 Effects of Intercell Interference and Correlated Shadowing 50

4.1 Motivation . . . . . . . . . . . . . . . . . . . 50

4.2 Intercell Interference Model . . . . . . . . . . . . . . . . . . . . 51

4.3 Correlated Shadowing Model . . . . . . . . . . . . . . . . 53

4.4 Capacity . . . . . . . . . . . . . . . . . . . . 55

4.5 Simulation Results . . . . . . . . . . . . . . . . . . . . . 58

4.6 Chapter Summary . . . . . . . . . . . . . . . . . 61

5 Autonomous Infrastructure Multihop Cellular Networks 63

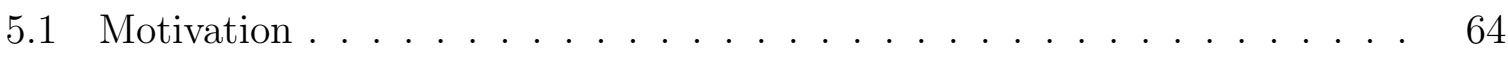

5.2 Autonomous Infrastructure . . . . . . . . . . . . . . . . . . . 65

5.2.1 Backbone Network Connections . . . . . . . . . . . . . 66 
5.2 .2 Access Point Criteria . . . . . . . . . . . . . . . 68

5.2 .3 Access Point Functionality . . . . . . . . . . . . . . . . . . 68

5.2 .4 Other Design Issues . . . . . . . . . . . . . . . . . . . . . . . . 69

5.3 Updated Network Model . . . . . . . . . . . . . . . . . . . . . . . . 70

5.4 Autonomous Infrastructure Multihop Cellular Network Design ..... 72

5.4.1 Integration of Access Points into Minimum Hop Routing Algorithm 73

5.4.2 Integration of Access Points into TDD-CDMA Mechanism . . . . 74

5.5 Capacity of the Autonomous Infrastructure Multihop Cellular Network . 76

5.6 Advantages of Autonomous Infrastructure . . . . . . . . . . . . . . 80

5.7 Modes of Operation . . . . . . . . . . . . . . . . . 81

5.8 Simulation Results . . . . . . . . . . . . . . . . . . . . . 84

5.9 Chapter Summary . . . . . . . . . . . . . . . . . . . . . . . . 88

6 Pilot Power Protocol for Optimal Transmission Range 90

6.1 Motivation . . . . . . . . . . . . . . . . . . . . . 91

6.2 Pilot Power Protocol for Autonomous Infrastructure Multihop Cellular Networks . . . . . . . . . . . . . . . . . . . . 96

6.3 Analytical Results . . . . . . . . . . . . . . . . . . . . . . . 100

6.4 Simulation Results . . . . . . . . . . . . . . . . . . . . 103

6.5 Optimality of Pilot Power Protocol . . . . . . . . . . . . . . . . . . 111

6.6 Chapter Summary . . . . . . . . . . . . . . . . . . . . . . . 112

$\begin{array}{llr}7 & \text { Conclusion } & 114\end{array}$

7.1 Summary . . . . . . . . . . . . . . . . . . . . . . 114

7.2 Concluding Remarks . . . . . . . . . . . . . . . . . . . . . . . 119

7.3 Future Research . . . . . . . . . . . . . . . . . . . . . 121

$\begin{array}{ll}\text { Bibliography } & 123\end{array}$ 


\section{List of Figures}

1.1 Cellular Networks, Ad hoc Networks and Multihop Cellular Networks . . 2

1.2 Bottleneck at the Base Station . . . . . . . . . . . . . . 6

2.1 Cluster of Cells in a Tradional Cellular Network . . . . . . . . . . . . . . 15

2.2 Spreading of a Data Signal in a CDMA Network . . . . . . . . . . . . . . 19

2.3 Interference in a CDMA Network . . . . . . . . . . . . . . . . 20

2.4 Despreading of a Data Signal in a CDMA Network . . . . . . . . . . . 20

2.5 Example of Routing in a Multihop Cellular Network . . . . . . . . . . . 28

3.1 Network Model for a Multihop Cellular Network . . . . . . . . . . . . . 38

3.2 Received Power at a Terminal . . . . . . . . . . . . . . . . . . . . 40

3.3 Communication Channels in a Multihop Cellular Network . . . . . . . . 42

3.4 Example of Minimum Hop Routing Algorithm for Layer 1 . . . . . . . . 43

3.5 Example of Minimum Hop Routing Algorithm for Layer 2 . . . . . . . . 44

3.6 Complete Routing Paths using the Minimum Hop Routing Algorithm . . 45

3.7 Use of Timeslots in a Multihop Cellular Network _ . . . . . . . . . . . . 47

3.8 Example of Packet Transmission using TDD in a Multihop Cellular Network 47

4.1 Intercell Interference in a Multihop Cellular Network . . . . . . . . . . . 52

4.2 Correlation of Shadow Fading . . . . . . . . . . . . . . . . . . 53

4.3 Average Intercell Interference vs Transmission Range Factor . . . . . . . 59

4.4 Average SINR vs Transmission Range Factor . . . . . . . . . . . . . . . . 60 
4.5 Total Power Consumption vs Transmission Range Factor . . . . . . . . . 61

5.1 Large Amounts of Interference at the Base Station . . . . . . . . . . . . . 64

5.2 Autonomous Infrastructure Multihop Cellular Network . . . . . . . . . . 72

5.3 Bad Timeslot Allocation for Terminals Transmitting to Access Points . . 75

5.4 Integration of Access Points into TDD-CDMA Mechanism for Autonomous Infrastructure Multihop Cellular Networks . . . . . . . . . . . . . . . . . 77

5.5 a) Regular Multihop Cellular Network b) Autonomous Infrastructure Multihop Cellular Network . . . . . . . . . . . . . . . . . . . 82

5.6 Average SINR vs Access Point Probability . . . . . . . . . . . . . . 85

5.7 Total Power Consumption vs Access Point Probability . . . . . . . . . . 86

5.8 Average Hop Count vs Access Point Probability . . . . . . . . . . . . . . 87

5.9 Average Uncovered Terminals vs Access Point Probability . . . . . . . . 88

5.10 Organic Growth of Network _ . . . . . . . . . . . . . . . . . 89

6.1 Percentage Covered Terminals vs Transmission Range Factor . . . . . . . 94

6.2 Average SINR vs Transmission Range Factor . . . . . . . . . . . . . . . . 95

6.3 Power Consumption per Mobile Terminal vs Transmission Range Factor . 95

6.4 Example Showing Loss of Connectivity . . . . . . . . . . . . . . . . . . . 97

6.5 Dividing Transmission Range into 3 Sectors . . . . . . . . . . . . . . . . 98

6.6 Distance Metrics in a Multihop Cellular Network: a) Ordinary Metrics b) Normalized Metrics . . . . . . . . . . . . . . . . . . . . . 101

6.7 Annulus Surrounding Normalized Distance $\bar{d}$. . . . . . . . . . . . . . . . 102

6.8 Chosen Transmission Range Factor vs Number of Mobile Terminals . . . 104

6.9 Percentage Covered Terminals vs Number of Mobile Terminals . . . . . . 105

6.10 Average SINR vs Number of Mobile Terminals . . . . . . . . . . . . . . . 106

6.11 Average Power Consumption vs Number of Mobile Terminals . . . . . . . 107

6.12 Average Hop Count vs Number of Mobile Terminals . . . . . . . . . . . . 108 
6.13 Percentage Covered Terminals vs Gamma . . . . . . . . . . . . . . . . 110 


\section{Chapter 1}

\section{Introduction}

In recent years, research has been performed in the area of multihop cellular networks due to their potential for higher capacity, lower energy consumption, and wider coverage [38][15]. The architecture of a multihop cellular network is similar to that of a traditional cellular network but differs in one significant way. In a multihop cellular network, the physical layer of mobile terminals is modified so that they can transmit not only to base stations but also directly to other mobile terminals. A mobile terminal can then reduce its transmission power and use other terminals as relays to enable its traffic to reach a base station. For this reason, the architecture of the multihop cellular network is similar to the ad hoc network in that transmissions from a source to a destination may require multiple hops. Figure 1.1 illustrates the differences between traditional cellular networks, ad hoc networks and multihop cellular networks.

Mobile terminals in multihop cellular networks use a smaller amount of transmission power on average than in traditional cellular networks because their maximum transmission range is reduced when relays are used to forward traffic to a base station [38]. Although more transmissions are required for the traffic to reach its destination, the reduction of path loss allows us to reduce the transmit power sufficiently so that the average 

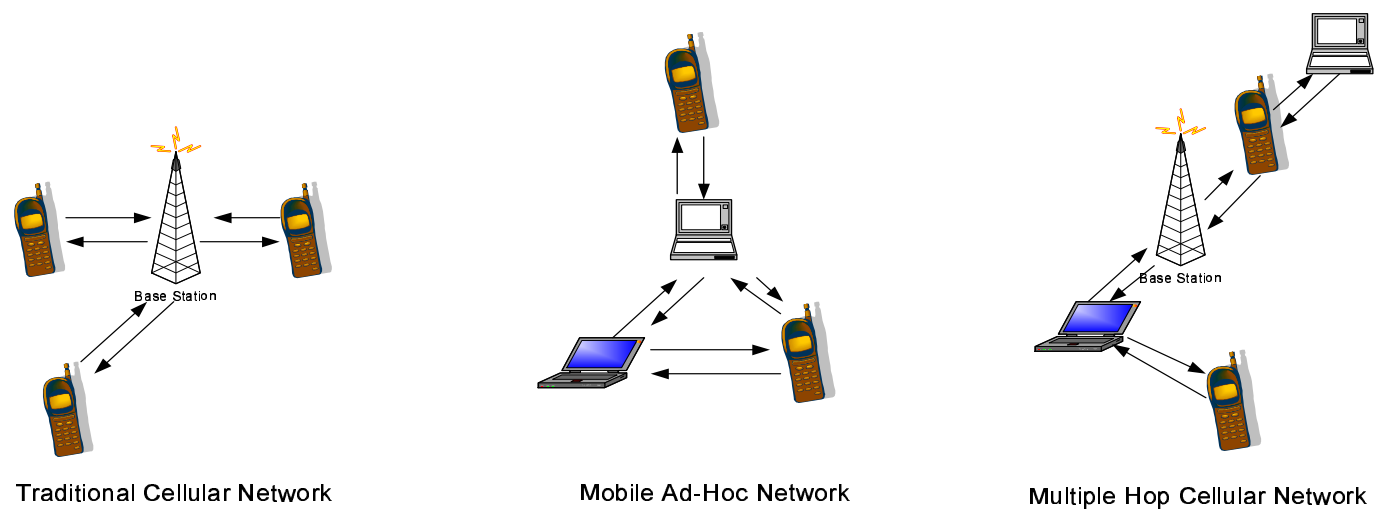

Figure 1.1: Cellular Networks, Ad hoc Networks and Multihop Cellular Networks

power consumption per terminal is lower. The majority of the intercell interference at a base station is caused by transmissions of mobile terminals that are near the boundaries of neighboring cells. Because the transmission power of terminals at the cell edge of neighboring cells is reduced in a multihop cellular network, the intercell interference may also be lower. The decrease in intercell interference in a multihop cellular network is what gives them the potential of higher Signal to Interference and Noise Ratios (SINR) than traditional cellular networks [15]. Moreover, the coverage in a multihop cellular network is wider because mobile terminals that are too far from a base station can still communicate with it by having their traffic relayed by other terminals. Coverage is also improved because a mobile terminal in a deep shadow fade with respect to the base station can still communicate with it by relaying its data to a nearby mobile terminal that is not in a deep fade.

\subsection{Motivation}

The rapid growth of wireless traffic has strengthened the demand for mobile communication networks with higher capacity, lower power consumption and greater coverage. Fourth generation wireless networks promise to meet these criteria while providing rich and diverse multimedia content requiring very high data rates. Cellular networks have evolved drastically from the 1st generation analog cellular networks of the early 1980's 
which used primarily FDMA, to the 2nd generation digital cellular networks of the 1990's which used TDMA and CDMA to the 3rd generation cellular networks in use today which use wideband CDMA for multiple access. One aspect that has not changed is the core architecture of the cellular network where a mobile terminal is required to transmit directly to a base station to have its traffic sent onto the backbone network towards its ultimate destination.

The traditional cellular network architecture may not have the flexibility to meet the requirements promised for $4 \mathrm{G}$ networks for the two following reasons. Firstly, fourth generation wireless networks promise to support transmission rates that are two orders of magnitude higher than those of $3 \mathrm{G}$ networks. The requirement of higher transmission rates is problematic because there is an inverse linear relationship between the transmission rate and the bit energy for a given transmission power level. Since the maximum Equivalent Isotropically Radiated Power (EIRP) levels of terminals are limited by regulatory governing bodies, the traditional cellular network may not be able to support these high data rates especially for terminals that are at the cell edge. Secondly, spectrum allocated to $4 \mathrm{G}$ networks is in many countries above the $2 \mathrm{GHz}$ band that is used in $3 \mathrm{G}$ networks. Higher carrier frequencies have less favorable radio propagation characteristics in that they are more vulnerable to non line-of-sight conditions which are common in urban cellular communication [46][78]. A potential solution to these problems would be to increase the density of base stations thus reducing the size of each cell and increasing the supported data rate of mobile terminals. However, this solution is disadvantageous because the deployment cost of base stations is very high and customers are unlikely to be willing to pay the same cost per bit for data traffic as they have been paying for voice $[46]$.

From this discussion it becomes clear that major modifications to the traditional cellular architecture are necessary in order to meet these requirements for future wireless networks. For this reason, an emerging architecture called the multihop cellular network 
has garnered a lot of attention from researchers as a potential candidate for future generation wireless networks [54][45]. Research shows that multihop cellular networks have the potential for higher capacity, lower energy consumption and wider coverage than traditional cellular networks [11][15]. The multihop cellular network architecture also provides a solution to the two aforementioned issues. Firstly, data rates for mobile terminals that are at the cell edge in multihop cellular networks are higher than in traditional cellular networks because the propagation loss between a mobile terminal and a relaying terminal is lower than the propagation loss between a mobile terminal and a base station. Secondly, multihop cellular networks are less vulnerable to non line-of-sight conditions that are problematic in networks using carriers above the $2 \mathrm{GHz}$ band. This is because mobile terminals can combat fading by choosing from a variety of different paths towards a base station created by relaying terminals whereas in a traditional cellular network mobile terminals are required to transmit directly to the base station [42].

\subsection{Problem Description}

Several novel problems are created in multihop cellular networks that did not exist in traditional cellular networks due to the modifications made to the network architecture and the changes made to the physical layer of mobile terminals. In a typical frequency division duplex (FDD) system, mobile terminals transmit on the low frequency band and receive on the high frequency band. In a multihop cellular system, mobile terminals require the capability to transmit to and receive from other terminals in addition to base stations. However, terminals cannot have simultaneous transmissions and receptions on the same frequency band due to the large power difference between transmitted and received signals [48]. To solve this problem, a time division duplexing (TDD) mechanism called TDD-CDMA was employed in [1][80], where mobile terminals would transmit on one timeslot and receive on the alternate timeslot in a manner that no mobile terminal 
would ever be simultaneously transmitting and receiving on the same band. In TDDCDMA, mobile terminals that are an even/odd number of hops away from a base station transmit on even/odd numbered timeslots and receive on odd/even numbered timeslots. Transmitting terminals will do so on the same frequency band using different spreading codes.

Another novel problem that exists in a multihop cellular network is that every mobile terminal is required to determine an efficient routing path to a base station. To solve this problem, several centralized routing algorithms have been proposed for multihop cellular networks in previous works [80][33]. However, in a real network it is unrealistic to assume that any terminal has an entire view of the network, and therefore a distributed routing algorithm is required. A distributed routing algorithm was proposed in [10] where minimum hop paths were found to a base station from every mobile terminal. Minimum hop routes were found to minimize the interference at the base station while maintaining a small overall energy consumption by mobile terminals in the network. However, the models used in these papers did not consider the effects of intercell interference on the overall capacity of the network. Furthermore, independent lognormal shadow fading coefficients from each terminal to the base station were used whereas experimental results show that shadowing coefficients are correlated [20]. In this thesis, the work in [10] and [11] is extended by taking the effects of intercell interference into account. This work is also extended by using correlated lognormal coefficients to model shadowing from each mobile terminal to the base station whereas [10] and [11] used independent lognormal coefficients.

Another problem that exists in a multihop cellular network is that the base station and the mobile terminals surrounding it are responsible for receiving and transmitting a large amount of traffic. This is because all traffic still either emanates or is destined to the base station causing a large congestion of interference near the base station making it the capacity bottleneck of the network [41][37][80]. Figure 1.2 illustrates the fact that 
the base station is the bottleneck of the system.

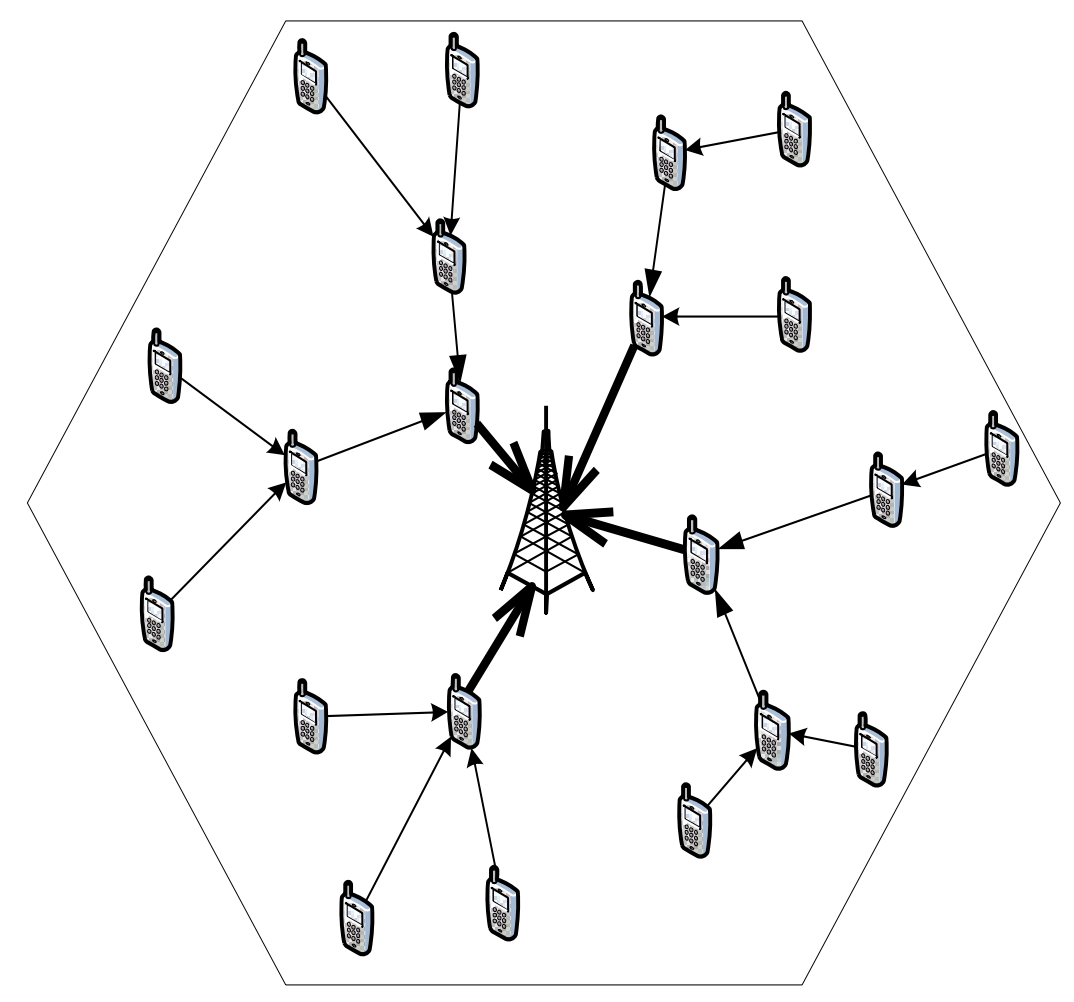

Figure 1.2: Bottleneck at the Base Station

Typically, when the congestion near the base station becomes large, a new base station would be deployed to help handle some of the traffic. However, the cost of construction of additional base stations is very high [30]. In this thesis, we present an alternate solution that is cost-effective, flexible and scalable to help alleviate the amount of traffic that base stations are required to handle. In this thesis, we propose a novel multihop cellular network architecture where users can connect their mobile terminals to the backbone network to become access points. Access points forward the traffic of other terminals directly onto the backbone network, as would a base station. We call this proposed architecture the autonomous infrastructure multihop cellular network. In this architecture network growth occurs organically because users can autonomously provide further links to the backbone network. This differs from other infrastructure based cellular networks where providing connections to the backbone network is solely the responsibility of the 
network operator.

A key feature of the proposed architecture is the automatic configuration of the transmission parameters of access points. In a traditional cellular network, radio frequency (RF) specialists monitor base station transmissions due to regulatory requirements and coverage and interference considerations. Because the exact locations of access points are unknown and can change frequently, it is not possible for RF specialists to adjust the transmission parameters of access points. Therefore another novel problem that exists is that access points have to determine their optimal transmission parameters autonomously.

An access point has a limited capability depending on the mobile terminal's battery power and the bandwidth of its connection to the backbone network and it is therefore only able to handle traffic for a limited number of terminals that are a certain number of hops away. An access point also has hardware limitations due to its power amplifier and its processing capability that restrict its maximum transmission power level. The access point will have to autonomously choose an optimal maximum transmission range that is high enough to ensure coverage of a sufficient number of terminals but not excessively high that it is causing too much interference. Clearly, the chosen maximum transmission range must involve the use of a transmission power level that is below the maximum transmission power level dictated by the hardware limitations. In our architecture, access points and the terminals they are serving have the same transmission range to ensure that bidirectional communication is possible. Having an optimally chosen transmission range is essential because it has a crucial effect on the number of hops required for traffic emanating from a terminal to reach the backbone network. This is because all terminals that receive and can decode an access point's pilot signal transmit directly to an access point and are required to act as relays for other terminals that do not receive an access point's pilot signal. The transmission range of access points and terminals is also instrumental in determining the percentage of terminals that receive coverage in a 
cell. Therefore determining an optimal maximum transmission range is essential as it plays a crucial role in the transmission patterns, interference levels, power consumption and coverage levels in a multihop cellular network.

An objective of this research is to develop a protocol that autonomously determines the optimal transmission range of access points and terminals for a given coverage requirement. In our architecture, an access point is restricted from transmitting data with a transmission power that is higher than its pilot power level. This implies that the transmission range of the access point is defined by its pilot power level. This is because only terminals that receive and can decode an access point's pilot signal transmit directly to that access point and are said to be within transmission range. This implies that we can meet our objective of optimizing the transmission range of mobile terminals by adjusting the pilot power levels of access points to optimal levels. In this thesis, we develop and present a pilot power protocol for the multihop cellular network which is used to determine optimal pilot power levels for both access points and base stations based on the density and spatial configuration of mobile terminals within a cell.

\subsection{Thesis Contributions}

In chapter 3 of this thesis, a complete system model of the multihop cellular network is presented. This includes the network model, the transmission and interference models, the communication channels and spreading codes, the routing algorithm used to form the multihop cellular network, the time-division duplexing CDMA mechanism and the power control mechanism.

In chapter 4 , we investigate the effects of intercell interference and correlated lognormal shadowing on system performance and incorporate these aspects into our system model. We compare our results to prior works on the multihop cellular network which had only considered one-cell systems where shadowing coefficients of different mobile 
terminals were modeled as being independent. The capacity on the reverse link of a CDMA cellular network depends on the total interference at the base station. The total interference at the base station is comprised of both in-cell interference and intercell interference. In a traditional cellular network, the in-cell and intercell interference typically account for two-thirds and one-third of the total interference in a cell, respectively. Our simulation results show that the intercell interference in a multihop cellular network is decreased by as much as $28.7 \mathrm{~dB}$ as compared to a traditional cellular network. This means that the intercell interference which accounts for one-third of the total interference in a traditional cellular network is effectively eliminated in a multihop cellular network. Simulation results in this thesis show that in a network modeled with correlated lognormal shadowing consisting of multiple cells, the average SINR at the base station is larger than that of a traditional cellular network by $1.6 \mathrm{~dB}$ as opposed to being smaller by $0.3 \mathrm{~dB}$ in a network consisting of one cell where correlated lognormal shadowing is not considered. An increase of $1.6 \mathrm{~dB}$ means that the multihop cellular network has a SINR that is $44 \%$ higher than that of a traditional cellular network due to the near elimination of intercell interference. Simulation results also show that in a network consisting of many cells where lognormal shadowing coefficients are modeled as being correlated, the overall transmission power consumption is lower than in a traditional cellular network by $23 \mathrm{~dB}$ as opposed to $18 \mathrm{~dB}$ in a one-cell system with independent lognormal shadowing coefficients.

In chapter 5 of this thesis, we propose a novel multihop cellular network architecture where users can connect their mobile terminals to the backbone network to give them the functionality of an access point so that they can forward the traffic of other terminals directly onto the backbone network. We call this proposed architecture the autonomous infrastructure multihop cellular network. This extends our previous work on autonomous infrastructure in traditional cellular networks and applies it to multihop cellular networks [66]. In this architecture, we assume that a subset of the mobile terminals in the network 
are built with an additional interface giving them the potential to transmit directly onto the backbone network. In the future, the cost of manufacturing these mobile terminals may become sufficiently inexpensive that a large percentage of terminals could have this capability. Users can connect their mobile terminal to the backbone network using this interface to improve capacity, coverage and data rates in a certain area. We refer to a mobile terminal as an access point when the terminal is using this interface to establish a direct connection to the backbone network and is able to handle traffic for other users, as would a base station. Access points can connect to the backbone network using a broadband wireless access network, a Local Area Network (LAN), a Digital Subscriber Line (DSL) connection, a Community Access Television (CATV) network or a Power Line Communication (PLC) connection. Depending on their locations, other terminals will be able to forward their traffic to a base station or to any access point that will then send the traffic directly onto the backbone network. This will lower the load on base stations and allow further links to the backbone network to be provided autonomously by the user. Allowing mobile terminals in a multihop cellular network to act as access points does not require any major modifications to the physical layer of mobile terminals because terminals already have the capability to transmit directly to one another. For this reason, the multihop cellular network architecture lends itself perfectly to having mobile terminals performing the functionality of access points. This is not the case in traditional cellular networks because mobile terminals only have the ability to transmit to base stations and cannot transmit directly to other terminals. Results of simulations and analysis presented in this dissertation show that our proposed solution leads to an increase in SINR at the base station, an increase in coverage and a decrease in overall power consumption as compared to a regular multihop cellular network.

In chapter 6 of this thesis, we develop and present a pilot power protocol for the autonomous infrastructure multihop cellular network. Although this algorithm was developed for use by access points in the autonomous infrastructure multihop cellular net- 
work, it can equally be used for base stations in both autonomous infrastructure and regular multihop cellular networks. The pilot power level of an access point is directly related to its transmission range and the pilot power protocol is used to determine its optimal transmission range. Access points and base stations are expected to function optimally in cells with either a high or low density of mobile terminals. They do not have any prior knowledge of the density or location of mobile terminals surrounding them and are required to autonomously set their pilot power levels to obtain a specified required coverage level while also obtaining as low power consumption and as high SINR as possible. To motivate the development of this algorithm we present simulation results indicating that the coverage within the network decreases as we decrease the transmission range. These simulation results also show that cells more densely populated by mobile terminals can handle lower transmission ranges than sparsely populated cells while still maintaining high coverage rates because there are a larger number of potential paths towards a base station or access point. However, simulation results also show that the SINR increases and the overall power consumption decreases as we decrease the transmission range. Therefore the pilot power protocol will have to address the tradeoff that exists between coverage versus SINR and power consumption. The pilot power protocol will be used by access points and base stations to determine their optimal pilot power level, thus their optimal transmission range. The algorithm autonomously increases the transmission range in sparsely populated cells to achieve the required coverage level and autonomously decreases the pilot power level in densely populated cells to improve SINR and power consumption while maintaining the required coverage level.

Simulation results of the pilot power protocol show that the chosen transmission range decreases as we increase the number of terminals within the network. Results of mathematical analysis presented in this thesis confirm and match our simulation results very closely. We also show that the bottleneck that exists at the base station in a regular multihop cellular network does not occur in the autonomous infrastructure multihop cel- 
lular network with the pilot power protocol because mobile terminals can be added to the network without decreasing the SINR at the base station. Furthermore simulation results show that when using the pilot power protocol the percentage of terminals that receive coverage remains roughly constant, independent of the density of mobile terminals. This feature is crucial for any multihop network as the coverage of a terminal is dependent not only on its distance from a base station but also on the number and location of mobile terminals surrounding it. Therefore by adjusting a parameter within the pilot power protocol, a required percentage of covered terminals can be specified without prior knowledge of the location or number of terminals within a cell which would be unknown in an autonomous infrastructure multihop cellular network. We also show analytically that the pilot power protocol achieves optimal values of SINR and power consumption for a given coverage requirement.

\subsection{Organization of Thesis}

The remainder of this thesis is organized as follows. Chapter 2 provides a summary of previous works in research areas that are closely related to the issues addressed in this thesis. Chapter 3 describes the system model of the multihop cellular network used in this thesis. Chapter 4 provides an analysis of the effects of intercell interference and correlated lognormal shadowing. Chapter 5 presents a novel architecture called the autonomous infrastructure multihop cellular network where mobile terminals can be connected to the backbone network to become access points. Chapter 6 details the pilot power protocol which can be used to determine optimal transmission ranges for access points, base stations and mobile terminals. Chapter 7 provides a conclusion and indicates some promising directions for future works based on the results of this thesis. 


\section{Chapter 2}

\section{Background and Related Literature}

The research presented in this thesis draws upon previous works in the fields of traditional cellular networks, ad hoc networks and multihop cellular networks. To obtain a clear understanding of this thesis, a concise overview of some essential background material is presented. We begin by describing aspects of the traditional cellular network such as the idea of breaking up the coverage area into cells as well as the evolution that these networks have experienced over the past few decades. We also review some fundamental properties of CDMA as it is the multiple access technology used in this thesis. We then discuss the aspects of ad hoc networks that are relevant to multihop cellular networks. Finally, we present a review of the previous research that has been performed on multihop cellular networks.

\subsection{Traditional Cellular Networks}

Traditional cellular networks made their first commercial appearance approximately 30 years ago. Since then, wireless cellular communication has experienced widespread growth throughout the whole world. According to the International Telecommunications Union of the United Nations, there were over 4.1 billion subscriptions to cellular phones as of the end of 2008 [70]. Cellular networks are designed to allow users to per- 
form wireless communications in a variety of situations. Users may be stationary, they may be moving slowly as would a pedestrian, or they may be moving at high speeds as would a user in a car. For this reason, the coverage area needs to be very large which has motivated the idea of cellular telephony.

\subsubsection{Cellular Structure}

In 1968, AT\&T Bell Laboratories proposed the concept of cellular telephony to the FCC [7]. The proposed idea focused on breaking up a large coverage area such as a city into many small coverage areas called cells. Figure 2.1 provides an illustration of the idea of cellular telephony by showing a cluster of cells in a traditional cellular network. A base station is located at the center of each cell and is responsible for handling the traffic of all mobile terminals within the cell. Base stations have a wireline connection to the Public Switched Telephone Network (PSTN) through the Mobile Switching Center (MSC) which is connected to the Base Station Controller (BSC) as shown in Figure 2.1 [69]. A fraction of the overall radio spectrum is allocated to each cell. Spectrum can be reused for cells that are far apart due to the power attenuation of radio signals that occurs with distance. This allows the network operator to service a greater number of customers using the same spectrum. Smaller cells also mean that base stations and mobile terminals will require lower power transmitters and smaller batteries. Because users are mobile, a handoff mechanism is necessary when a user moves from one cell's coverage area into another. Efficient handoff schemes have been developed so that mobile coverage is ubiquitous across cells and handoff occurs without the user's knowledge, thus allowing calls to be uninterrupted [71][2].

\subsubsection{Evolution of Traditional Cellular Networks}

Cellular networks have evolved through four different stages since their first inception in the late 1970's. Each of these stages is typically referred to as a generation of cellular 


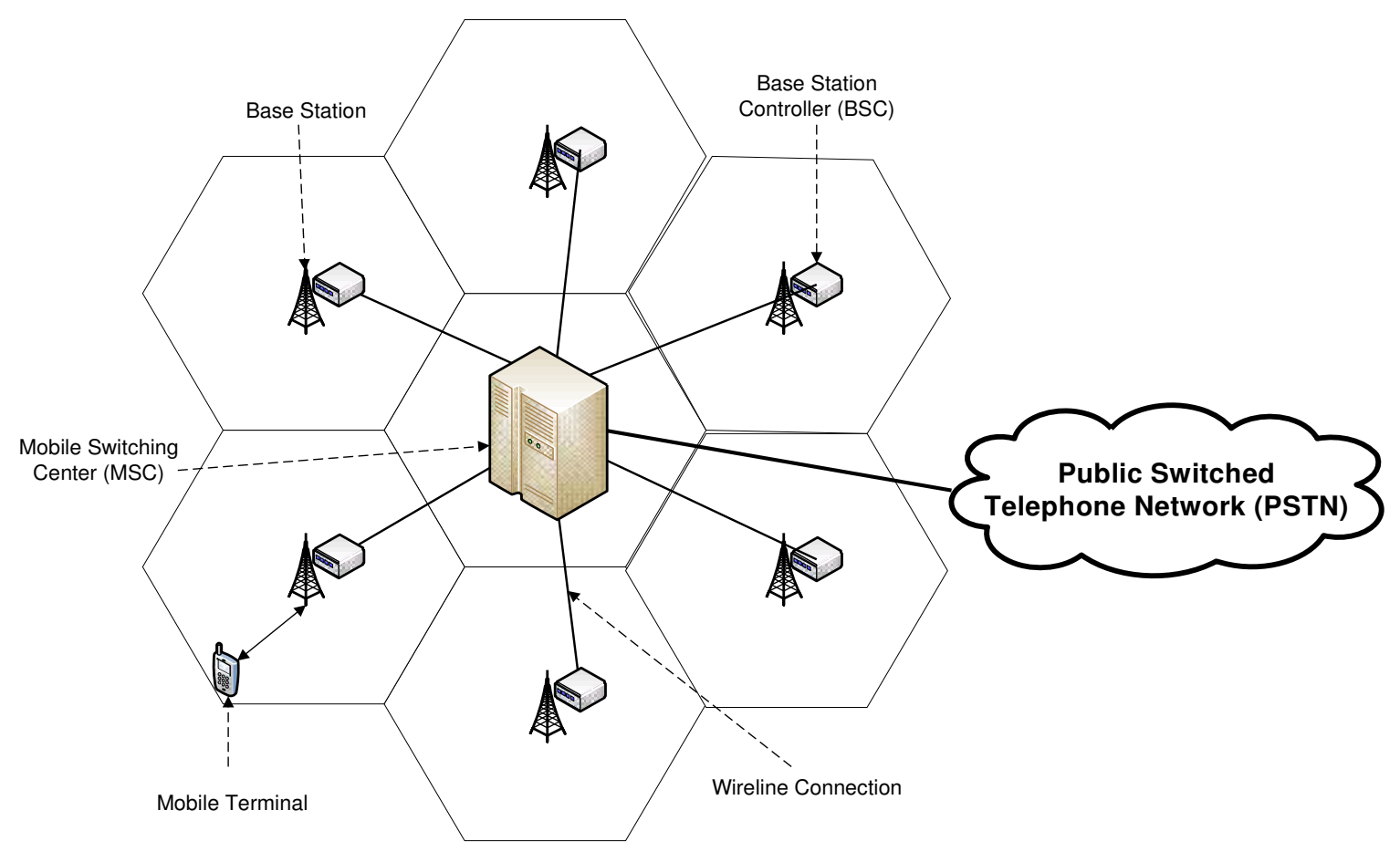

Figure 2.1: Cluster of Cells in a Tradional Cellular Network

networks.

The first generation $(1 \mathrm{G})$ of traditional cellular networks began with the first operational commercial deployment of a cellular network by Nippon Telephone and Telegraph in Japan in 1979. This system used 600 frequency modulated duplex channels each having a bandwidth of $25 \mathrm{kHz}$ in the $800 \mathrm{MHz}$ band. Other $1 \mathrm{G}$ networks include the Nordic Mobile Telephone System in Scandinavia in 1981, the Total Access Communication System in the United Kingdom in 1982 and the Advanced Mobile Phone System (AMPS) in the United States [7][69]. A common feature of all these $1 \mathrm{G}$ networks is that they were analog systems that relied on frequency modulation. Although these networks provided the ability to perform wireless voice communication, the networks did not support a large number of users due to inefficient spectrum utilization.

The need for a second generation $(2 \mathrm{G})$ of cellular networks became apparent in the late 1980's as analog communication was becoming obsolete. Advances in integrated circuit design made digital communication not only more practical but also less costly 
than analog technology. Digital communication allowed for a much more efficient use of spectrum reducing the bandwidth required for voice and data transmission. $2 \mathrm{G}$ systems can be classified by the multiple access scheme employed: Frequency Division Multiple Access (FDMA), Time Division Multiple Access (TDMA) or Code Division Multiple Access (CDMA). In FDMA, the spectrum is divided into different frequency bands and each user is assigned a frequency band upon which to transmit. In TDMA, several users transmit on the same frequency band but do so on different timeslots. CDMA employs spread spectrum techniques to achieve multiple access. In CDMA, several users transmit on the same frequency band at the same time but they modulate their transmissions with high bandwidth spreading codes that have low cross-correlation.

The commercial deployment of $2 \mathrm{G}$ systems began in 1990 with the deployment of the Global System for Mobile communications (GSM) in Europe. GSM which uses TDMA brought a single standard to European cellular telephony. GSM was later updated to support packet switching through the General Packet Radio Service (GPRS). In North America, two 2G systems co-existed. In 1992, digital cellular was introduced to the United States with IS-54 using TDMA for multiple access. In 1993, IS-95 was also deployed in the United States using CDMA as its multiple access technique. CDMA networks had an increased network capacity and greater spectral efficiency than networks based on TDMA and FDMA [7].

The third generation $(3 \mathrm{G})$ of cellular networks was envisioned so that higher data rates would be supported that would allow Internet browsing, video-calling and other multimedia applications on mobile terminals. $3 \mathrm{G}$ networks rely on a data-centric packet switched network as opposed to the solely connection-oriented network of prior generations [69]. All of the $3 \mathrm{G}$ networks that have been deployed rely on CDMA for multiple access. The first commercial deployment of $3 \mathrm{G}$ occurred in Japan with the deployment of W-CDMA by NTT DoCoMo in 2001. An alternative technology known as CDMA2000 1xEV-DO was deployed in the United States by Verizon Wireless in 2003 [77]. 
Currently, the Third Generation Partnership Project (3GPP) and the Institute of Electrical and Electronics Engineers (IEEE) are in the process of developing the fourth generation (4G) of wireless communication systems. 4G systems promise to support wireless broadband access, mobile TV, HDTV and video chat. The technical requirements for $4 \mathrm{G}$ are data rates of $100 \mathrm{Mbps}$ for users that are moving at high speeds and 1 Gbps for users that are relatively stationary. 4G systems will also strive for greater spectral efficiency (in bits/s/Hz), more simultaneous users per cell and improved coverage. 4G systems are also expected to be solely packet based leading to an all Internet Protocol (IP) network. The two beyond 3G networks that are currently being designed, tested and deployed are called the Long Term Evolution (LTE) of 3GPP [53] and IEEE 802.16 WiMAX [44]. However these standards still fall short of achieving the data rates that are required for $4 \mathrm{G}$ especially for terminals located near the cell edge. For this reason, researchers are currently developing new 4G standards called LTE-Advanced [54][50] and IEEE 802.16j-2009 [45] that use relaying as a way to meet the data rate and coverage requirements of $4 \mathrm{G}$.

\subsubsection{CDMA Cellular Networks}

CDMA is the multiple access technology that is used in our model of the multihop cellular network. CDMA is used because it is the underlying technology in 3G systems and a large amount of resources have been put towards the deployment of CDMA and 3G networks throughout the world. Furthermore, CDMA has a higher spectral efficiency than FDMA and TDMA. Also, CDMA may perform better than Orthogonal Frequency Division Multiple Access (OFDMA) in a multihop cellular network because mitigating the effects of co-channel interference is more difficult in OFDMA than in CDMA [21]. This is because OFDMA requires dynamic channel allocation with specialized coordination between neighboring base stations. Because relays are used in multihop cellular networks which leads to an increased number of transmissions by nearby terminals, the 
interference averaging effect of CDMA may make it better suited for multihop cellular networks than OFDMA. However, the results presented in this thesis are also applicable to networks using other multiple access techniques that are robust to interference such as OFDMA. This includes the proposed autonomous infrastructure multihop cellular network architecture presented in chapter 5 and the pilot power protocol presented in chapter 6 .

\section{Spread Spectrum}

A CDMA system is a direct sequence spread spectrum system where many terminals transmit on the same frequency band simultaneously. The bandwidth of the data signal is spread using a Pseudo-Noise (PN) code that is not correlated with the data signal. As a result, the bandwidth of the spread signal becomes much higher than the bandwidth of the data signal [52].

A number of advantages of spread spectrum are :

1. Interference Rejection: Since the signal is spread over a large frequency band, the power spectral density becomes very small and a small amount of interference is created at any given frequency.

2. Multiple Access Capability: Multiple users can use the same frequency band simultaneously.

3. Reduction of Effects of Multipath Fading: Spread spectrum signals are less vulnerable to multipath fading due to the inherent frequency diversity obtained by spreading a signal over a wide frequency range.

4. Anti-Jamming Capability: Because the data is spread over a wide frequency band, a very high powered wide-band signal is required to jam a spread spectrum signal. 
Figure 2.2 depicts the power spectral density of a data signal before and after it has been spread by a PN-code [17]. It can be observed that the power spectral density of the data signal becomes spread over a wide bandwidth due to the high frequency of the PN-code.

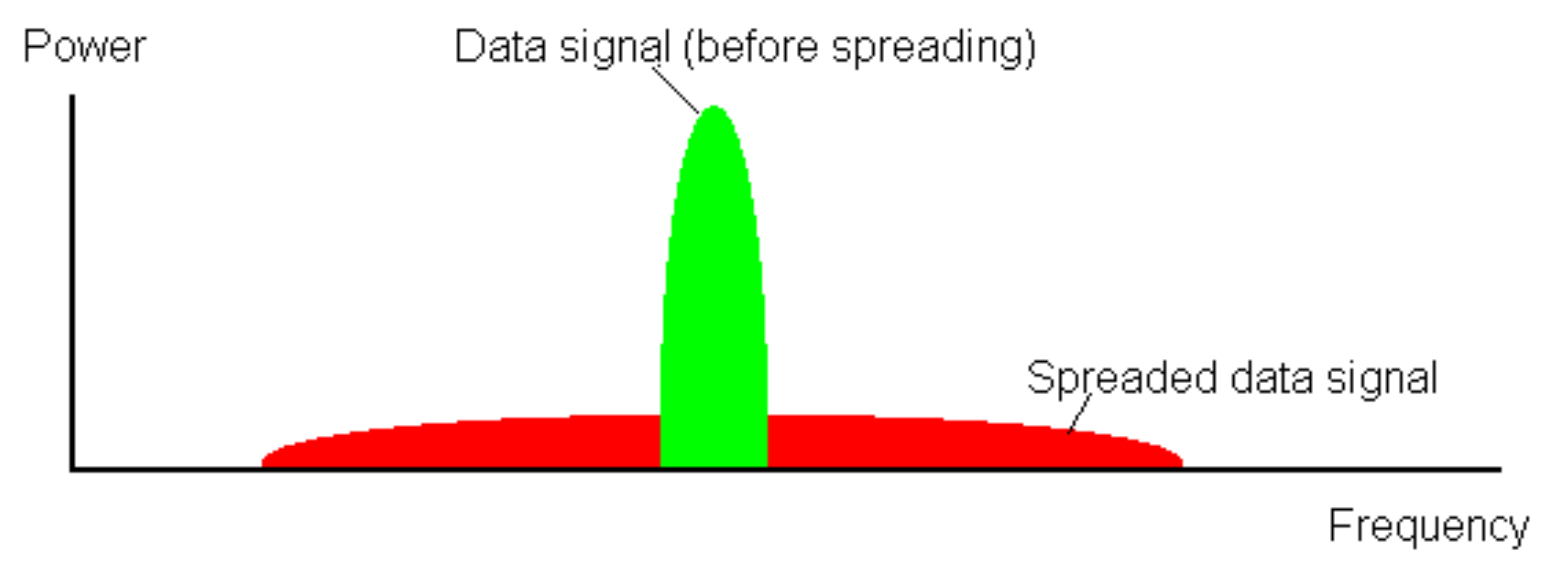

Figure 2.2: Spreading of a Data Signal in a CDMA Network

Figure 2.3 shows the power spectral density of the spread spectrum signal with respect to some narrow-band interference. This figure shows that a narrow-band interference, which would have greatly interfered with an unspread data signal, only provides limited interference to the spread spectrum signal.

Figure 2.4 shows the data signal and the interference once it has been despread at the receiver. This figure shows us that while the data signal gets despread, the interference is spread out and that only a limited amount of noise passes through the low pass filter. This explains why spread spectrum systems have good interference rejection and antijamming properties. 


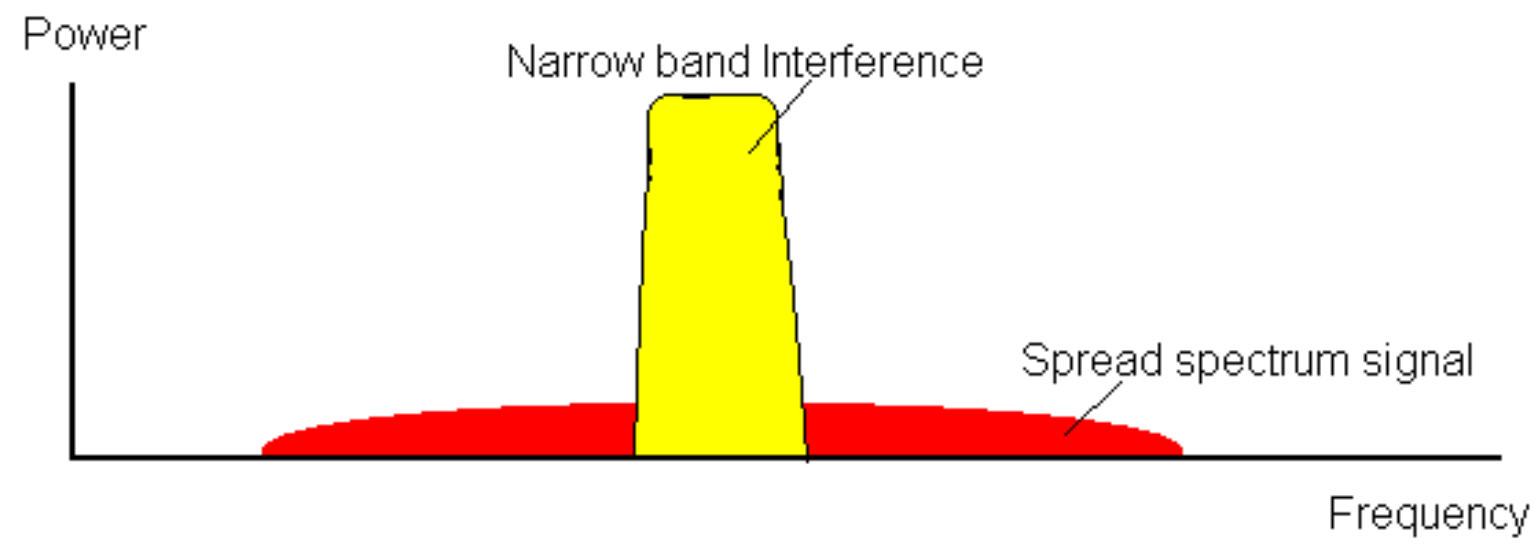

Figure 2.3: Interference in a CDMA Network

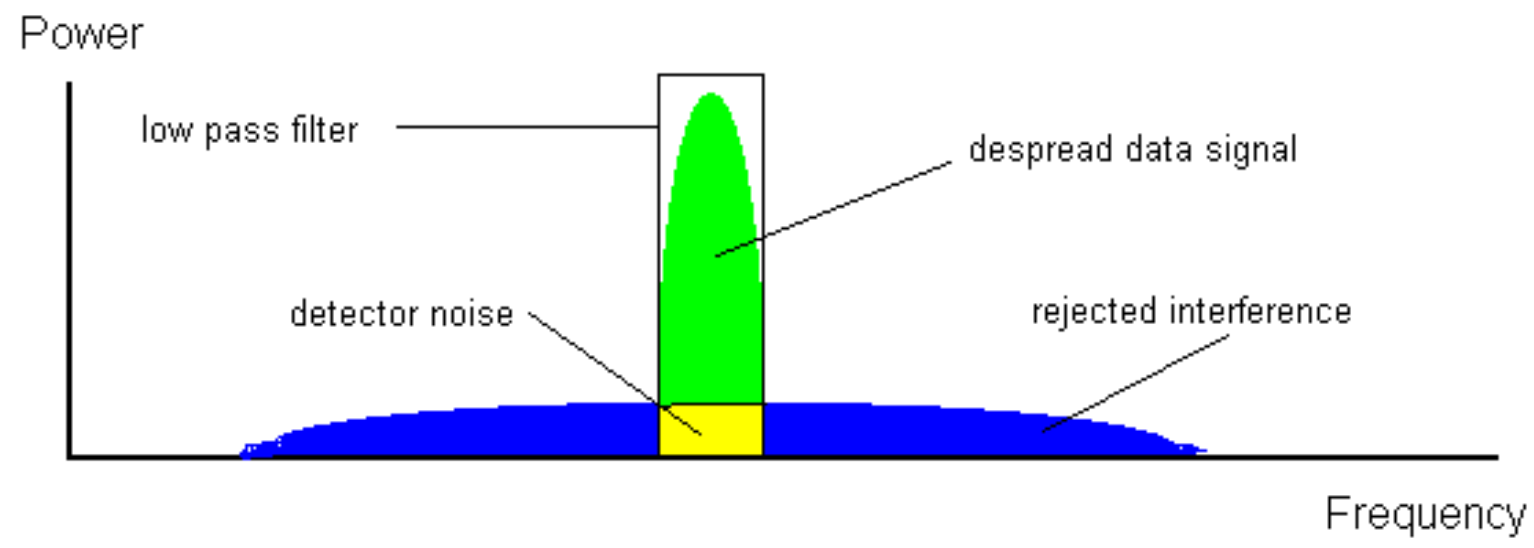

Figure 2.4: Despreading of a Data Signal in a CDMA Network 


\section{Spreading Codes}

In a CDMA network each user is assigned a particular PN-code which is used to spread its data signal. There are several properties that a PN-code should possess in order for it to be used as a spreading code in a CDMA system [67].

1. Different PN-codes should have low cross-correlation to minimize the interference between different users allowing more mobile terminals to be supported by the network.

2. PN-codes should be balanced in order to avoid having a DC-component in the spread data signal.

Spreading codes that are used in practical systems are: Walsh-Hadamard codes, msequences, Gold-codes and Kasami-codes. Walsh sequences [3] are orthogonal while the other sequences have non-zero cross-correlation values [18] [59] [62].

Orthogonal codes have zero cross-correlation but only when there is no offset between the codes. In fact, they tend to have large cross-correlation values when there is an offset which can be caused by a lack of synchronization between transmitters. Therefore, orthogonal codes tend to be used only in perfectly synchronized environments such as on the forward link of the cellular system.

Non-orthogonal spreading codes are used when synchronization is not possible such as on the reverse link of a cellular network. Because spreading codes are not orthogonal, transmissions from other users in CDMA are treated as interference. Despite this interference, users are still able to decode incoming signals as long as the ratio of the incoming signal power over the interference power and noise, called the Signal to Interference and Noise Ratio (SINR), is above a certain threshold. For this reason, CDMA networks are considered to be interference limited [36]. 


\section{Power Control}

The purpose of power control is to minimize multiple access interference by attempting to maintain a constant SINR at the base station from each transmitting terminal by adjusting their transmitter power levels. There are two primary types of power control mechanisms. The first type is known as open loop power control where mobile terminals adjust their power based on the power of the pilot signal received from the base station. One drawback of this mechanism is that fading is dependent on frequency and the amount of fading on the forward and the reverse link may differ. Therefore, the power level for transmissions on the reverse link may be adjusted incorrectly by observing the fading that is occurring on the forward link. The second type of power control mechanism is known as closed loop power control which relies on a feedback channel between the base station and the mobile terminal. This mechanism provides tight power control where the power level can be adjusted by $1.0 \mathrm{~dB}$ every $0.67 \mathrm{~ms}$. Both open loop and closed loop power control are used on the reverse link of a W-CDMA network [51].

\section{Capacity}

One of the primary objectives in mobile communications is to increase the maximum number of mobile terminals that can use a network at a given data rate while maintaining a satisfactory bit error rate (BER). The BER of the reverse link of a CDMA network is a function of the received SINR at the base station.

The probability of bit error in a CDMA system is a monotonically decreasing function of the SINR:

$$
\begin{gathered}
P_{e}=f(S I N R) \\
P_{e}=f\left(\frac{E_{b}}{N_{0}+I}\right)
\end{gathered}
$$


where $E_{b}$ is the energy per bit of the received data signal, $N_{0}$ is the power spectral density of thermal noise, and I is the equivalent power spectral density of the multiple access interference caused by other mobile terminals.

In a single cell system when there are M users transmitting in the system this becomes:

$$
P_{e}=f\left(\frac{E_{b}}{N_{0}+\frac{\Psi}{N} \sum_{k=1}^{M-1} E_{b, i}}\right)
$$

where

$$
\Psi=\frac{N^{2}}{E_{b}^{2} T_{c}} \int_{-\infty}^{\infty}|H(f)|^{4} d f
$$

$\mathrm{N}$ is the processing gain, $E_{b, i}$ is the energy per bit for the i-th user, $H(f)$ is the Fourier transform of the chip pulse, and $T_{c}$ is the chip size. Note that $\Psi=1$ for a sinc chip pulse.

If $N_{0}$ is small and all signals have the same energy per bit at the receiver due to power control, this equation simplifies to:

$$
P_{e}=f\left(\frac{N}{\Psi(M-1)}\right)
$$

If the SINR at the receiver has to be greater than $\alpha$ to obtain a required BER, the system can support M users where:

$$
M=\frac{N}{\Psi \cdot \alpha}+1
$$

This formula only takes into account a single cell system. When a system involving many cells is considered, it can be shown that intercell interference decreases the maximum number of users $\mathrm{M}$ that can be supported. This can be modeled by multiplying $\mathrm{M}$ by a factor $\mathrm{F}$ (typically $\approx \frac{2}{3}$ ). In addition, if we are discussing a communication system where speech is being transmitted, on average each voice circuit is only active for a fraction $\mathrm{V}$ of the total time (typically $\approx 0.4$ ). Taking this into account we can update 
our capacity equation, so that the maximum number of users that can be supported at a given $\mathrm{SINR}=\alpha$ is:

$$
M=\frac{N \cdot F}{\Psi \cdot \alpha \cdot V}+1
$$

\subsection{Ad Hoc Networks}

An ad hoc network is a multihop wireless network where a collection of mobile terminals form a temporary network without any centralized infrastructure or wireline backbone. The main feature that distinguishes these networks from the conventional wireless cellular network is that an ad hoc network does not rely on an expensive centralized base station. For this reason, ad hoc networks are rapidly deployable and self-organizing. Transmissions between terminals in an ad hoc network may require multiple hops and therefore all terminals work together to ensure successful packet delivery from the source to the destination. Terminals in an ad hoc network may be either stationary or mobile resulting in a dynamic topology requiring frequent routing path updates.

Initial research on ad hoc networks was primarily conducted for military applications where rapid network creation and survivability are of the utmost importance. Relying on a centralized base station is impractical and often impossible in a hostile environment. This is because the deployment of a base station is usually not possible and the network is subject to failure if the centralized infrastructure is destroyed. The Defense Advanced Research Projects Agency (DARPA) of the United States' Department of Defense played a crucial role in the advancement of ad hoc networks with their research and deployment of the Packet Radio Network (PRNet) in 1972. The rise in popularity of the Internet in the 1990's as well as the reduction in the cost of computers and radio technology such as IEEE 802.11 wireless local area networks and Bluetooth, have created a resurgence in research on ad hoc networks [58]. 
Ad hoc networks are inexpensive and rapidly deployable due to their lack of infrastructure. Because terminals cooperate and relay each other's transmissions, mobile terminals require low power transmitters allowing them to remain small in size and conserve battery power. Transmission rates are high because transmissions only occur between terminals that are close to one another.

\subsubsection{Limitations}

Despite the aforementioned advantages of ad hoc networks, they also suffer from several limitations. The following drawbacks often limit the deployment of ad hoc networks to smaller scale networks where coverage of only a small area is required.

Channel assignment in a typical CDMA cellular system is achieved by the assigning of spreading codes to mobile terminals by a base station. Channel reuse is obtained by reusing the same channels in different cells. However, due to a lack of centralized infrastructure, a more difficult distributed approach is used in ad hoc networks where the same channels are reused for mobile terminals spaced sufficiently far apart. Typical channel assignment schemes tend to aggregate terminals into clusters and use an algorithm to allocate different channels to terminals within a cluster [24][16]. However, channels have to be reallocated frequently due to network topology changes caused by the mobility of terminals which leads to a waste of network resources.

The absence of a base station in an ad hoc network means that mobile terminals have to cooperate with each other in order for packets to be transmitted from one terminal to another. Each mobile terminal only has a low power transmitter and intermediate terminals may be required to relay traffic along a routing path towards the destination. Several routing algorithms have been proposed in previous works [26][75][49] but without any centralized infrastructure to help with coordination, these algorithms tend to be complex and use a substantial amount of limited resources. Because nodes are mobile, frequent routing updates may be required leading to a waste of network resources. 


\subsection{Multihop Cellular Networks}

Multihop cellular networks were envisioned to solve many of the limitations that are characteristic of ad hoc networks while maintaining their advantages. By allowing multihop communications on top of a cellular infrastructure, many of the drawbacks of traditional cellular networks can also be mitigated. Multihop cellular networks have been shown to increase SINR and coverage as compared to a traditional cellular network with an overall decrease in power consumption by mobile terminals [15][25]. Mobile terminals in a multihop cellular network transmit over smaller distances than in a traditional cellular network which makes higher bit rates possible while maintaining a low EIRP transmission power. Multihop cellular networks can also provide load-balancing among base stations and help the network recover from base station failures [74][56]. Multihop cellular networks also solve the ad hoc network's problems of inefficient channel assignment and routing due to the presence of a centralized base station. The presence of base stations allows multihop cellular networks to be used in networks where a large coverage area is required as opposed to ad hoc networks that can only cover a limited area.

\subsubsection{Network Architecture}

Multihop cellular networks attempt to benefit from the advantages of traditional cellular networks and ad hoc networks by utilizing properties from each of these networks. In this hybrid network, transmissions between mobile terminals and base stations are performed using multiple hops. Mobile terminals (or dedicated relay stations) are required to relay traffic for other terminals to the base station. Because mobile terminals and base stations are no longer required to be in direct transmission range of one another, their transmission power can be reduced and they can be designed to have lower power transmitters.

A cell in a multihop cellular network is defined differently than in a traditional cellular network because transmissions from a mobile terminal can no longer necessarily reach the 
base station in a single hop. In a multihop cellular network, a cell is defined as one base station and all of the mobile terminals that transmit to that base station using multiple hops if necessary. The reverse link is defined as the transmissions emanating from a mobile terminal having the base station as their final destination. The forward link is defined as the transmissions emanating from a base station having a mobile terminal as their final destination.

The transmission range of each mobile terminal is decreased in a multihop cellular network and therefore traffic cannot necessarily be transmitted from a mobile terminal to a base station in a single hop. Figure 2.5 shows an example of how routing occurs in a multihop cellular network. Suppose that mobile terminal A wants to transmit a packet to mobile terminal B that is in a different cell. A will first forward the packet towards its base station using multiple hops. Once the packet arrives at the base station, it will transmit the packet to the base station of the destination terminal, using its connection to the backbone network. This illustrates how a multihop cellular network reduces the hop count of a packet as compared to an ad hoc network when the source and destination are far apart. Communication between terminals that are far apart would be extremely inefficient and suffer from a high delay, large power consumption and high routing complexity which limits the size of ad hoc networks. Mobile terminal B's base station will then forward the packet to B using multiple hops.

\subsubsection{Limitations}

Despite the advantages related to multihop cellular networks, there are also several difficulties that pose problems to researchers and network designers limiting the actual deployment of multihop cellular networks. Multihop cellular networks have a much higher system complexity than traditional cellular networks. Issues such as handoff, routing and channel allocation are more complex in multihop cellular networks than in tradi- 


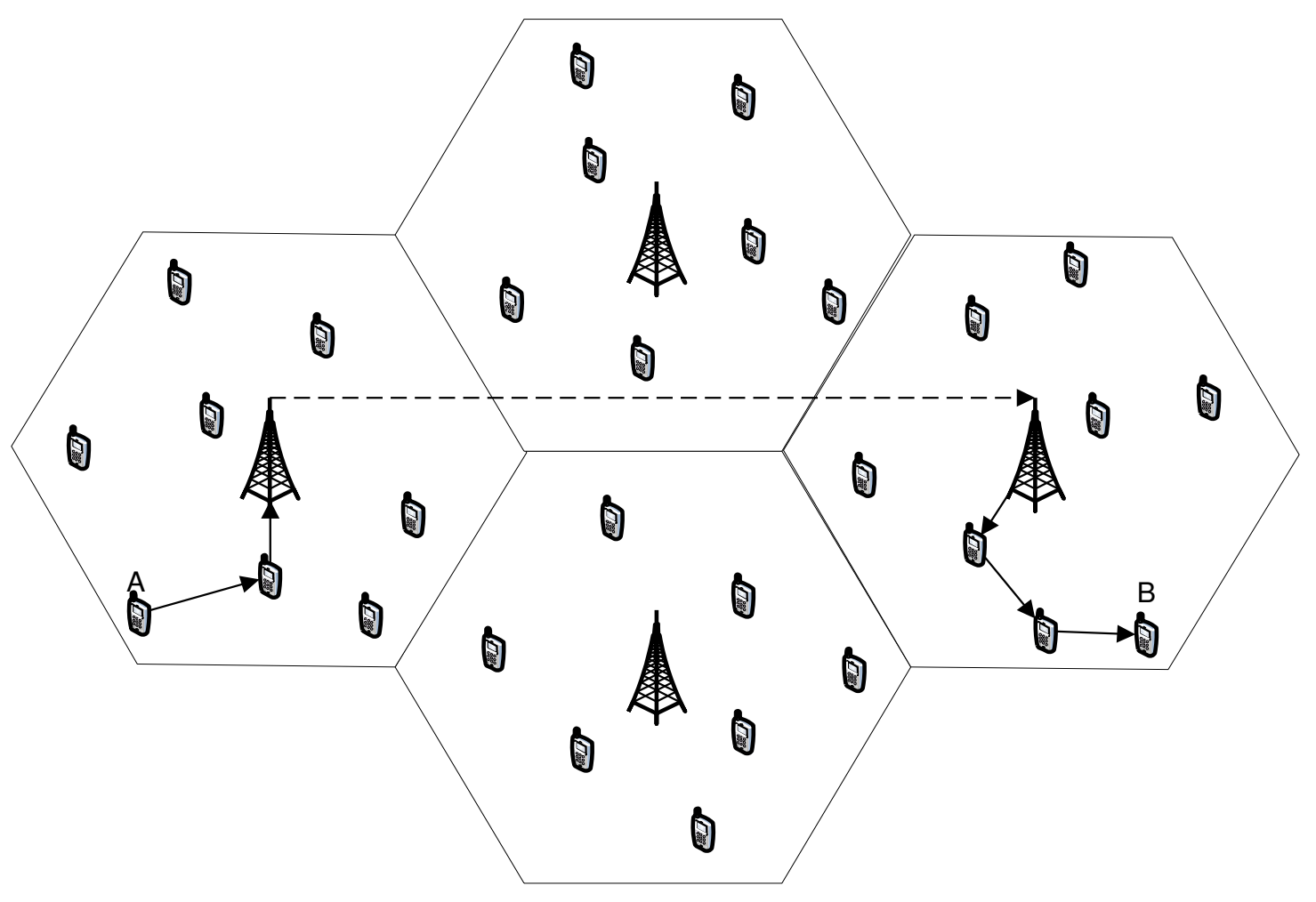

Figure 2.5: Example of Routing in a Multihop Cellular Network

tional cellular networks because transmissions now occur between mobile terminals and relaying terminals as well as between mobile terminals and base stations. For example, handoff will occur more often because it can be triggered by the mobility of the relaying mobile terminal or the mobility of the transmitting terminal. The overhead created by the calculation and updating of routing paths as well as by the occurrence of handoff may limit the improvements in capacity of multihop cellular networks that have been found in previous research [57][37]. Mobile terminals will also require increased processing capability when they are used as relays to allow them to handle traffic for other users. The base station as well as the relays will also be required to have a database to store routing path information.

Delay is also an issue that is more severe in multihop cellular networks than in traditional cellular networks. Because traffic is transmitted using multiple hops, the endto-end delay will be higher than when transmissions occur directly to the base station. 
Delay will increase as we decrease the transmission ranges of the terminals and more hops are required for traffic emanating from a terminal to reach the base station.

Multihop cellular networks have also potentially weaker security than traditional cellular networks. This is because intermediate relaying terminals receive data from mobile terminals and are required to forward it to the base station. These intermediate terminals could be perpetrators of tampering or fraud especially if transmissions involve financial transactions.

\subsubsection{Variations of Multihop Cellular Networks}

Many different variations of architectures and protocols have been proposed for multihop cellular networks in previous research. State of the art research on multihop cellular networks can be classified by the following options [36]:

1. Relaying can be performed by other mobile terminals or it can be performed by fixed relay stations that are pre-installed by the network operator.

2. Relaying can be performed on the same frequency band used by mobile terminals for regular transmissions or it can be performed on an additional frequency band.

3. Terminals can use multihop relaying where traffic is relayed along a single path towards the destination or they can use cooperative relaying where traffic is transmitted along multiple paths using cooperative diversity techniques to attempt to exploit spatial diversity.

4. Relays can decode the desired signal and then re-encode and forward it towards the destination (decode-and-forward) or they can act as repeaters by simply amplifying the received signal and transmitting it towards the destination (amplifyand-forward). 
Relaying stations can be fixed and pre-installed by the network operator or relaying can be performed by other mobile terminals. Fixed relay stations have the advantage of being stationary which results in less frequent routing updates. Fixed relay stations are cheaper than normal base stations because they do not require a wireline connection to the backbone network and are only required to receive incoming traffic and forward it towards the destination. However, deployment of several of these fixed relay stations in each cell still requires a significant capital investment by the network operator. Employing mobile terminals as relays is advantageous because the performance gains of multihop cellular networks can be achieved without having to invest in the infrastructure of fixed relay stations. Employing mobile terminals as relays is especially beneficial when node density is high and traffic load is low because there are likely to be many idle mobile terminals available to relay data. Mobile terminals acting as relays have to expend additional power to relay data but this power is low due to the decreased transmission range of terminals. Therefore, in spite of the power required to relay data, the overall transmission power consumption in a multihop cellular network is lower than in a traditional cellular network [36]. For these reasons the architecture designed in this thesis employs mobile terminal relays.

Relaying can be performed on the same frequency band used by mobile terminals for regular transmissions or it can be performed on an additional frequency band, termed in-band and out-of-band relaying respectively. An advantage of in-band relaying is that mobile terminals do not have to be modified to have the ability to transmit on a different frequency band, leading to a smaller and more inexpensive mobile terminal. Mobile terminals that are either idle or transmitting at a low bit rate can act as relays for other terminals. In a CDMA network mobile terminals in different hops along the routing path can share the same frequency band using different spreading codes transmitting using Time Division Duplexing (TDD). Multihop cellular networks that use this technique are referred to as TDD-CDMA multihop cellular networks [36]. Out-of-band relaying 
relies on mobile terminals having at least two radios or interfaces and transmitting on two different frequency bands. With out-of-band relaying, mobile terminals can use the alternate radio interface solely to perform relaying or they can even use the alternate interface for their regular transmissions when they experience bad channel conditions on the primary frequency band. The architecture designed in this thesis employs inband relaying as it places less restrictions on the design of the mobile terminals and the availability of additional spectrum.

Terminals can use multihop relaying where traffic is relayed along a single path towards the destination or they can use cooperative relaying where traffic is transmitted along multiple paths using cooperative diversity techniques to attempt to exploit spatial diversity. The use of multihop relaying gives structure to the network because a routing path between each mobile terminal and the base station is determined upon which traffic can be transmitted. This is advantageous because only a limited number of terminals are required to forward traffic along a known path leading to less interference. The advantage of the cooperative diversity technique is that a virtual antenna array is formed by multiple terminals in the network leading to spatial diversity. Research has shown that the use of cooperative diversity may increase capacity and coverage of the network [63]. Diversity may be easier to obtain using multiple terminals as opposed to placing multiple antennas on a single small terminal as in a typical Multiple-Input-MultipleOutput (MIMO) system [6]. However, a disadvantage of this technique is that it requires a much larger number of overall transmissions to obtain this diversity. This may lead to an increase in interference within the network and an increase in power consumption. Although the relative merits of these two schemes is still an open research problem that is attracting a large amount of attention from the research community, a recent paper that compared these two schemes showed that a higher network throughput is obtained when using multihop relaying with a single routing path than when using cooperative relaying with multiple paths [27]. The architecture designed in this thesis uses multihop 
relaying with a routing algorithm that finds a single path between every terminal and a base station.

Relays can use decode-and-forward where they decode the desired signal and then reencode and forward it towards the destination. Relaying using decode-and-forward can improve system capacity in interference limited environments because the interference and noise accumulated in the transmission from the source to the relay can be eliminated when the transmission is correctly decoded at the relay. Alternatively, relays can use amplify-and-forward where relays amplify the received signal and transmit it towards the destination. The advantage of this scheme is that relays using amplify-and-forward are less complex than those using decode-and-forward. However, the basic problem with this scheme is that relays amplify not only the desired signal but also the interference and noise. Therefore, amplify-and-forward works best in limited interference and noise scenarios and has lesser benefits in the presence of heavy interference [4][43]. For these reasons, the architecture designed in this thesis uses decode-and-forward relaying.

\subsubsection{Previous Works}

Several multihop cellular network architectures propose the use of mobile terminals as relays [37]. These schemes include Lin and Hsu's MCN [38], ODMA [1], Ad Hoc Cellular (A-Cell) [60], Unified Cellular and Ad hoc Network (UCAN) [40], Pervasive Ad Hoc Relaying for Cellular Systems (PARCelS) [81], Self Organizing Packet Radio with Overlay (SOPRANO) [80] and the Minimum Hop Routing Multihop Cellular Network [10][11]. These schemes differ in their approach, the multiple access schemes used as well as the assumptions that are made in their research. One of the first works on multihop cellular networks was performed by Lin and Hsu [38] as an architecture that would maintain the benefits of traditional cellular networks while incorporating the flexibility of ad hoc networks. This paper compared the throughput of a multihop cellular network with that of a single hop cellular network. This paper used a Request To Send/Clear To 
Send (RTS/CTS) method for multiple access and only required the base station to be used for intracellular calls. Their simulations showed that the overall capacity of the multihop cellular network was higher than that of a traditional cellular network when a large percentage of traffic was destined to mobile terminals within the same cell. This is because the bottleneck that would normally occur at the base station was decreased because it was no longer required to handle intracellular traffic. Other early research on integrating multihop relaying with cellular networks was performed by the 3GPP under the name Opportunity Driven Multiple Access (ODMA) [1]. In ODMA, TDD is used to separate transmissions by mobile terminals on different hops of the routing path.

Further research on the multihop cellular network was performed with the design of an architecture called SOPRANO which used CDMA as the multiple access method [80][48]. In [48], a centralized SIR/Power Routing Algorithm similar to Dijkstra's algorithm was proposed in an attempt to minimize a network cost function. Research was also performed by Kusuma in which he presented a centralized routing algorithm that found the minimum power routing paths for all nodes in the network [33]. However it is not realistic to assume that an entire view of the network is known and therefore a distributed routing algorithm is required. A distributed routing algorithm was proposed in [10][11] where minimum hop routes between every mobile terminal and a base station were found. The advantage of using minimum hop routing is that it minimizes the interference at the base station while maintaining a low overall energy consumption. However, the models that had been used in previous works did not consider networks consisting of multiple cells where intercell interference affects the capacity of the network. Moreover, independent lognormal fading coefficients from each terminal to the base station were used whereas experimental results show that fading coefficients are correlated [20]. In chapter 4 of this thesis, we extend the work in [10][11] by incorporating intercell interference and correlated shadowing into our model. We analyze how these factors affect the SINR at the base station and the overall energy consumption in a multihop cellular 
network formed using the minimum hop routing algorithm.

There are also many proposed architectures that make use of fixed relay stations [37]. These schemes include integrated cellular and ad hoc relaying (iCAR) [74] and the virtual multihop cellular network (VMCN) [32]. In iCAR dedicated relay stations are deployed by the network operator at the edges between each pair of cells. These relay stations use an additional frequency band separate from the ones used by mobile terminals for transmissions and provide load balancing by diverting traffic from congested cells to cells with less traffic. In VMCN, virtual cells are formed by a mobile terminal and a number of fixed relay stations. Mobile terminals are allowed to communicate with multiple relay stations simultaneously to benefit from spatial diversity.

There are also several architectures that make use of both fixed relay stations and mobile terminal relays [37]. These networks include Hierarchical Multihop Cellular Network (HMCN) [39] and Mobile-Assisted Data Forwarding (MADF) [76].

After making a thorough examination of all of these previous works on multihop cellular networks, we identified an issue that has yet to be thoroughly addressed. The problem is that the base station and the relaying terminals surrounding it are responsible for receiving and forwarding a large number of transmissions. This is because all traffic either emanates from or is destined to the base station causing a large congestion of interference near the base station making that area the capacity bottleneck of the network. Typically, when this congestion becomes large, a new base station would be deployed. However, the cost of additional base stations is very high. In chapter 5 of this thesis, we present an alternate solution that is cost-effective, flexible and scalable to help alleviate the amount of traffic that base stations are required to handle. We propose a novel multihop cellular network architecture where users can connect their mobile terminal to the backbone network to become an access point and forward the traffic of other terminals directly onto the backbone. This proposed architecture is called the autonomous infrastructure multihop cellular network. A key feature of this architecture is the autonomous 
configuration of the transmission parameters of access points and base stations. In chapter 6 of this thesis, we present a pilot power protocol for both access points and base stations that autonomously configures their transmission ranges to achieve optimal levels of SINR and power consumption for a given coverage requirement. 


\section{Chapter 3}

\section{Multihop Cellular Network System}

\section{Model}

In this chapter we present the system model of the multihop cellular network that is used in the remainder of this thesis. This system model will be slightly modified in subsequent chapters to incorporate autonomous infrastructure but all of the aspects of the system model presented in this section will continue to be relevant. We begin by introducing the network model and the propagation model. We continue by providing details of the spreading codes and presenting the various communication channels that are used by mobile terminals and base stations. We also discuss the routing algorithm that is used to find paths between every mobile terminal and a base station. The TDD-CDMA mechanism is also presented which allows mobile terminals in different hops along the routing path to share the same frequency band but transmit using different spreading codes and using time division duplexing. We then present the power control mechanism as well as the parameters that are used in the simulations in this thesis. 


\subsection{Network Model}

In our model of the multihop cellular network, we break up the whole coverage area into smaller coverage areas that are approximated using hexagonal cells. A base station is located at the center of each cell. Therefore, each base station has 6 neighboring base stations. For the remainder of this thesis, the multihop cellular network is modeled using a central reference cell as well as its six neighboring base stations. All of our results are obtained from the reference cell with neighboring cells being treated solely as sources of interference as seen in Figure 3.1. We assume that all cells use the same frequency band leading to a frequency reuse factor of 1 . Circular cells are used instead of hexagonal cells in analysis and simulations for the sake of mathematical tractability and this has been shown to be a good approximation [34]. A number of mobile terminals $M$ is randomly distributed in each cell of radius $R$. Each mobile terminal in the reference cell is referred to by a value from $i=1, \ldots, M$ and the base station has value $i=0$. In a traditional cellular network the maximum transmission range $r$ of base stations is $R$ which is the range required to cover an entire cell. In a multihop cellular network the maximum transmission range $r$ of mobile terminals and base stations is lower than in a traditional cellular network by a factor of $K$ which is a parameter called the transmission range

factor and $r=\frac{R}{K}$. Therefore, as we increase $K$, we decrease the transmission range of terminals and increase the number of hops required for a packet from a given mobile terminal to reach a base station.

Mobile terminals are employed to relay traffic using a Decode-and-Forward technique on both the forward and the reverse links. This is because multihop relaying has been shown to increase capacity and transmission rates on the forward link in addition to the reverse link [8][35]. Although it is possible for transmissions on the forward and the reverse link to share the same frequency band, our model assumes the use of two independent frequencies for transmissions in each direction as in [80][79]. Performance evaluation is performed primarily on the reverse link because the traffic concentration 


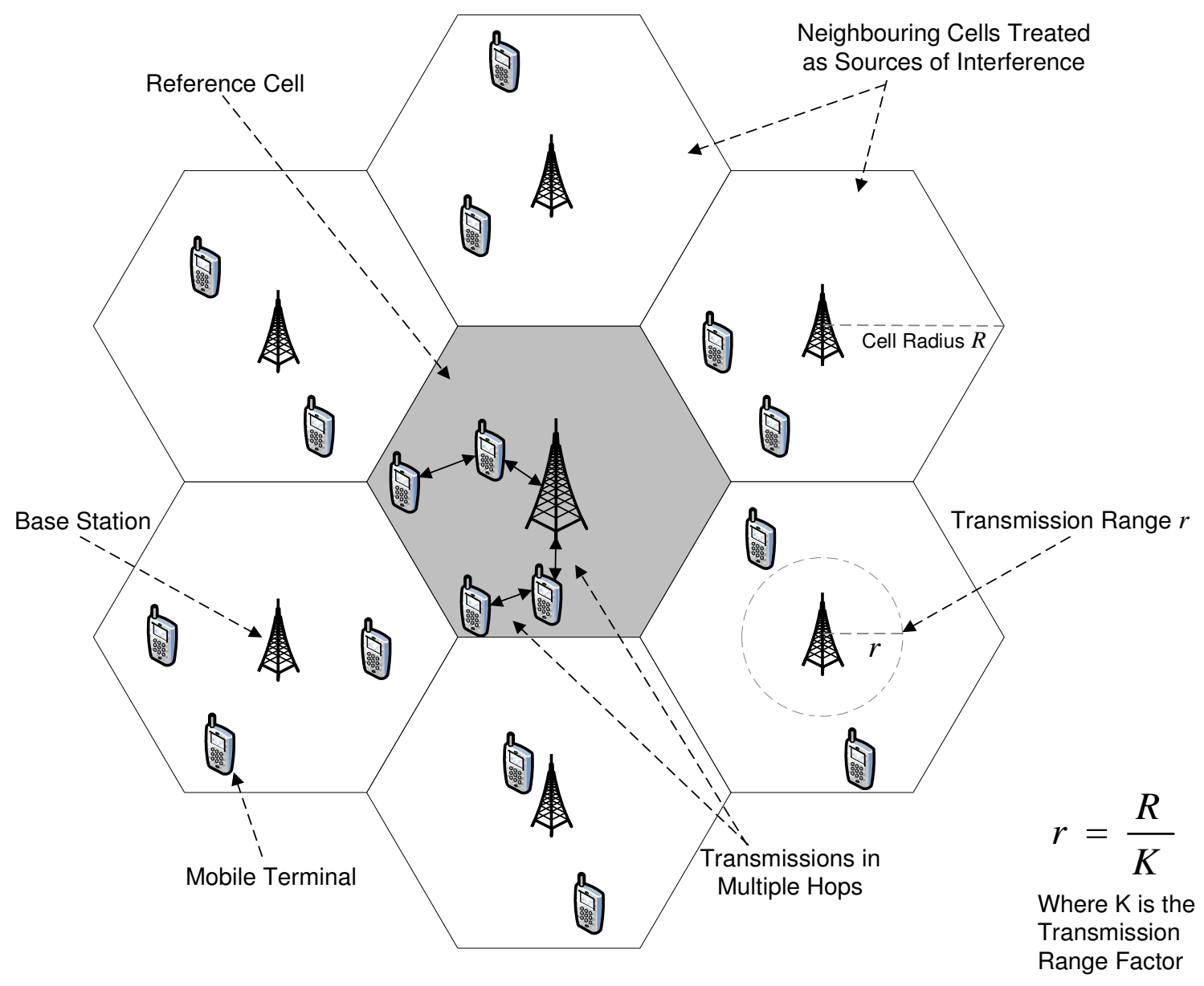

Figure 3.1: Network Model for a Multihop Cellular Network

and interference is highest at the base station as it is the bottleneck of the network [9]. Furthermore, power consumption by terminals is higher on the reverse link than on the forward link because both regular mobile terminals and relaying terminals are required to transmit on the reverse link whereas only relaying terminals are required to transmit on the forward link. Mobile terminals and base stations are assumed to have one omnidirectional antenna. Regular transmissions from mobile terminals as well as those for the purpose of relaying are performed on the same frequency band (in-band relaying). CDMA is used as the multiple access technique. Minimum hop routing paths are used to connect mobile terminals to base stations using mobile terminals as relays as described in section 3.5. Mobile terminals in different hops along the routing path share 
the same frequency band in code domain using time division duplexing (TDD-CDMA) as described in section 3.6.

\subsection{Transmission Model}

Suppose mobile terminal $i$ transmits with power $P_{i}$ to relaying mobile terminal $j$ towards the backbone network. The path loss $G_{i j}$ between terminals $i$ and $j$ is

$$
G_{i j}=d_{i j}^{-l}
$$

where $d_{i j}$ is the distance between terminals $i$ and $j$, and $l$ is the path loss exponent. The effect of lognormal shadowing $\gamma_{i j}$ between terminals $i$ and $j$ is

$$
\gamma_{i j}=10^{\xi(0, \sigma)} / 10
$$

where $\xi_{(0, \sigma)}$ is a Gaussian distributed random variable with zero mean and standard deviation $\sigma$.

The received power $P\left(d_{i j}\right)$ at terminal $j$ which is a distance $d_{i j}$ away from transmitting terminal $i$ is

$$
P\left(d_{i j}\right)=P_{i} \cdot G_{i j} \cdot \gamma_{i j}=P_{i} \cdot d_{i j}^{-l} \cdot 10^{\xi_{(0, \sigma)} / 10}
$$

Mobile terminal $j$ is said to be within transmission range of terminal $i$ and can correctly decode its transmission when

$$
\mathrm{SINR}_{i j}=\frac{P\left(d_{i j}\right)}{N_{0}+\frac{I_{j}}{N}}>\alpha
$$

where $N_{0}$ is the additive white gaussian noise power, $I_{j}$ is the co-channel interference, $N$ is the processing gain and $\alpha$ is a threshold required to decode the transmission with low error probability. Figure 3.2 illustrates how a transmission is made from terminal 1 
to the base station using terminals 2 and 3 as relays along with the received powers at each terminal.

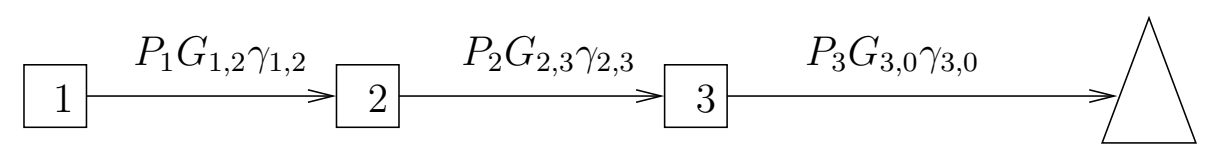

Figure 3.2: Received Power at a Terminal

\subsection{Spreading Codes}

Spreading codes are the essential mechanism that allow CDMA networks to obtain a higher capacity than networks using other forms of multiple access like FDMA and TDMA. As discussed in chapter 2, there are two distinct types of spreading sequences known as orthogonal sequences and non-orthogonal sequences. Orthogonal sequences are used in perfectly synchronized environments such as the forward link of a traditional cellular network. Non-orthogonal sequences, on the other hand, are used in non-synchronized environments such as the reverse link of a traditional cellular system.

In a multihop cellular network, the base station is in charge of distributing spreading codes to mobile terminals. As a perfectly synchronized environment is impossible to create, a spreading code scheme comprised of non-orthogonal spreading code sequences is used. This is true both for transmissions from a mobile terminal on the forward link and the reverse link. In a traditional CDMA system, non-orthogonal codes are used only on the reverse link. The reason that orthogonal codes can be used on the forward link in a traditional CDMA system but not in a multihop cellular network is that in a traditional cellular network, all transmissions on the forward link emanate only from the base station. This differs in a multihop cellular network because in this system, every mobile terminal may also have to transmit on the forward link.

With asynchronous CDMA there are usually enough spreading code sequences to be 
assigned to all mobile terminals within a cell. In our multihop cellular network architecture, each mobile terminal is therefore assigned a unique non-orthogonal spreading code sequence by the base station. In fact, terminals that have to forward packets for other terminals may be assigned several spreading codes. Relaying terminals are able to simultaneously receive from multiple terminals. They also have the ability to simultaneously transmit multiple packets to a single receiver. Interference caused by other transmissions is treated as independent additive noise [72].

\subsection{Communication Channels}

Because mobile terminals in addition to base stations are now transmitting on the forward link, the communication channels allocated in a multihop cellular network are dependent on the type of transmission, forward link or reverse link, as opposed to the type of hardware, base station or mobile terminal. A mobile terminal relay has to act like a base station of a traditional cellular network by providing synchronization information and paging data to the downstream mobile terminals that transmit to it. Therefore, all base stations and relaying mobile terminals have pilot, sync, paging and traffic channels for transmissions on the forward link and receptions on the reverse link. All relaying mobile terminals and regular mobile terminals have access and traffic channels for transmissions on the reverse link and receptions on the forward link. Figure 3.3 shows the different types of communication channels that are allocated to different terminals in a multihop cellular network. Note that transmissions in the forward and reverse directions between two terminals occur during different timeslots as will be explained in section 3.6.

\subsection{Routing Algorithm}

The minimum hop routing algorithm proposed in [10][11] was found to minimize interference in a multihop cellular network while maintaining a low overall energy consumption. 

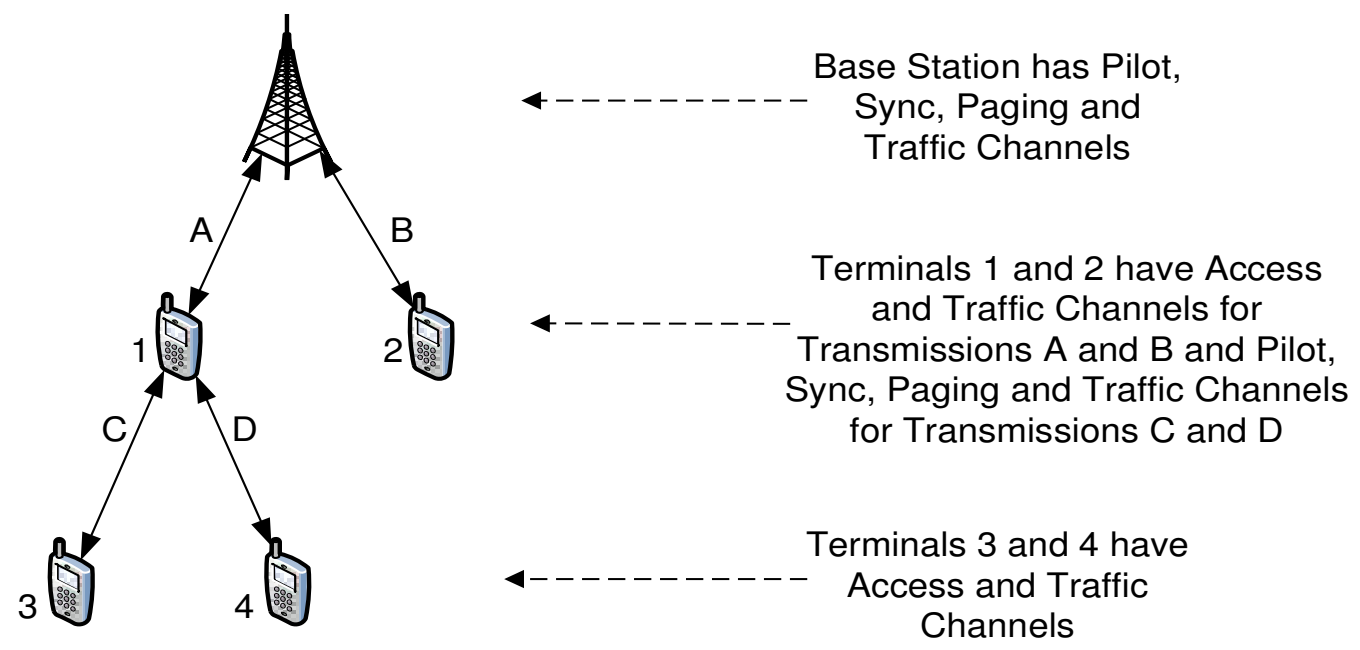

Figure 3.3: Communication Channels in a Multihop Cellular Network

For this reason, minimum hop routing is used in our architecture. The following is a brief overview of the minimum hop routing algorithm. Initially, when a network of mobile terminals is activated, they are completely unaware of their locations. Routing paths need to be found from all mobile terminals in a cell to the base station. The route discovery process of the minimum hop routing algorithm begins at the base station. The base station will transmit its pilot signal with a transmission range $r$ that would be its regular transmission range required to cover the cell $R$, divided by the transmission range factor $K$. All mobile terminals that correctly receive this pilot signal will be said to belong to layer 1 because they are within one hop of the base station. These mobile terminals will also be referred to as the children of the base station and the base station will be referred to as the parent of these mobile terminals. The mobile terminals will then reply to the base station to inform it that they are within its transmission range. The base station will then send a message to the mobile terminals in layer 1, assigning them a timeslot and a spreading code. Figure 3.4 shows an example of the minimum hop routing algorithm showing how route discovery occurs for terminals in layer 1 . Note that in reality the circle around the base station indicating layer 1 will not have a perfectly circular shape due to lognormal shadowing as well as the existence of varying amounts of interference 
at different locations.

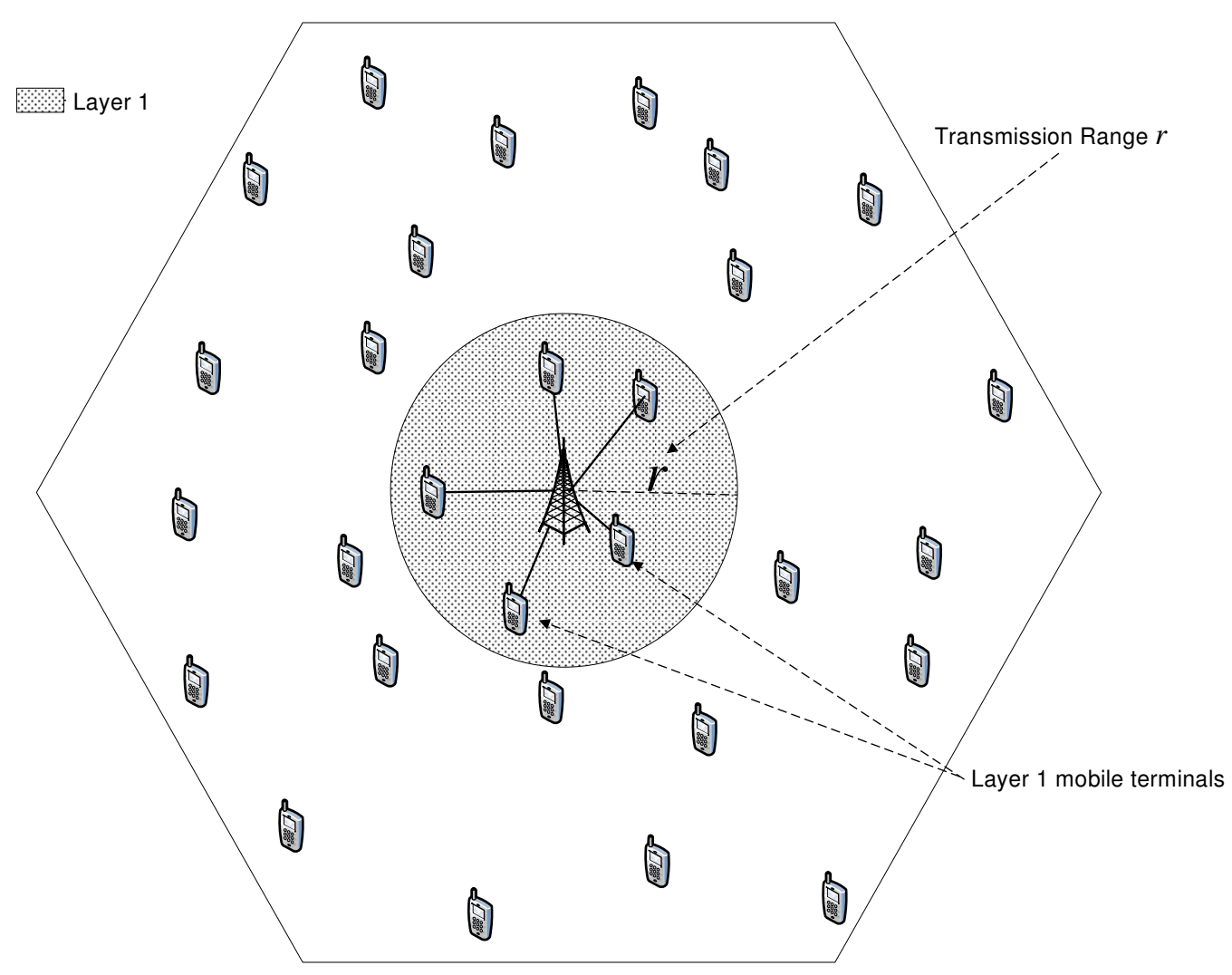

Figure 3.4: Example of Minimum Hop Routing Algorithm for Layer 1

The routing paths for the remaining mobile terminals, that are not within transmission range of the base station, still need to be computed. To accomplish this, each mobile terminal in layer 1 will transmit a pilot signal on its assigned timeslot to all mobile terminals within its range. Mobile terminals only broadcast their pilot signal during a small percentage of a timeslot with a random start time. This is to minimize interference created by multiple pilot signals. Any mobile terminal that is not part of layer 1 , that correctly receives a pilot signal from a terminal in layer 1 , will become part of layer 2. After finishing scanning all of the received pilot signals, each mobile terminal in layer 2 will choose the terminal in layer 1 with the highest received pilot power to be its parent. Parent terminals will also inform the base station about the existence of their children using their spreading codes. The base station will then assign spreading codes 
and timeslots to terminals in layer 2 and will forward this information to the parents of the terminals in layer 2 who will in turn forward the information to the mobile terminals in layer 2. Figure 3.5 shows our example of the minimum hop routing algorithm showing how route discovery occurs for terminals in layer 2 .

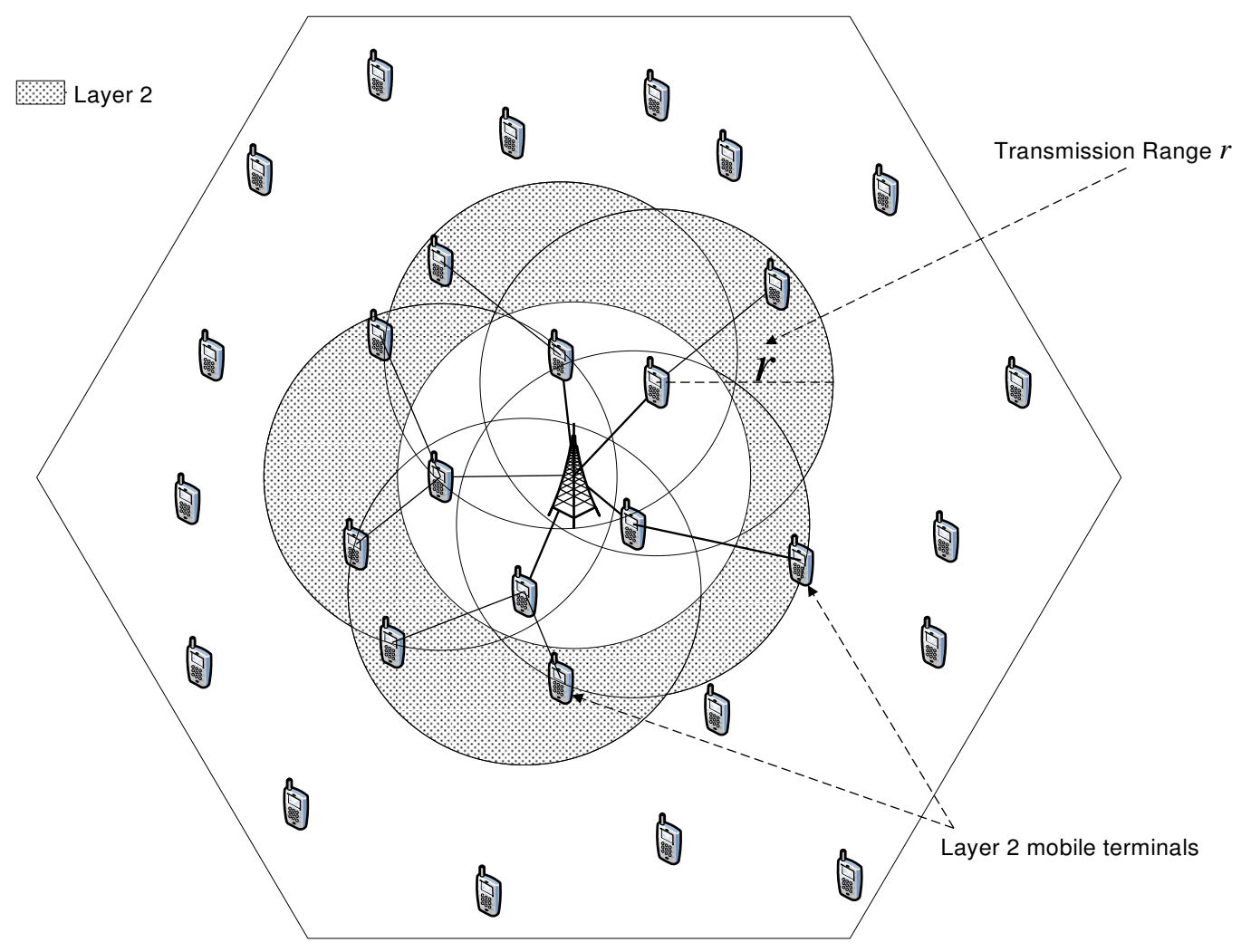

Figure 3.5: Example of Minimum Hop Routing Algorithm for Layer 2

Repeating this algorithm for mobile terminals in higher layers, complete routing tables can be calculated for the entire multihop cellular network. Figure 3.6 shows the routing paths for all terminals in the cell found using the minimum hop routing algorithm.

Links can break at any time due to the motion of mobile terminals or because of changing amounts of interference between a parent and a child. Therefore routing path changes should be made when a more suitable parent terminal is available [29]. Whenever a mobile terminal detects a pilot signal that is stronger than the pilot signal of the terminal to which it is currently transmitting, a handoff will occur and the routing path 


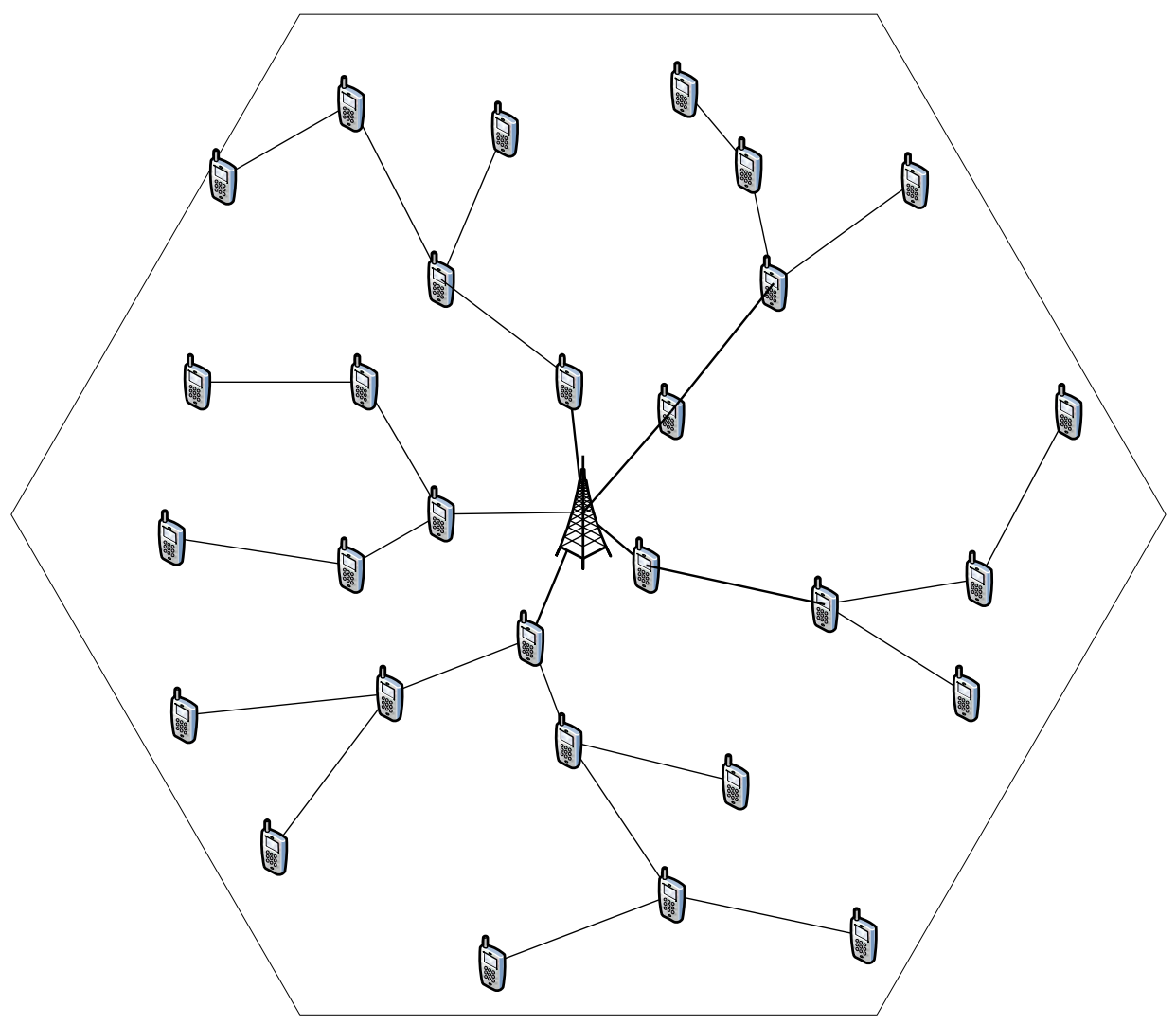

Figure 3.6: Complete Routing Paths using the Minimum Hop Routing Algorithm

will change. Full details on how routing path changes are handled for a multihop cellular network with minimum hop routes are provided in [11]. The routing algorithm can also function so that a mobile terminal that chooses not to act as a relay or that has a low remaining battery power does not transmit a pilot signal so that it is not chosen as a relay. This could lower the number of times that the routing paths need to be updated.

\subsection{Time Division Duplex CDMA}

Due to the high power difference between signals being transmitted and signals being received at a terminal, it is impractical for a terminal to have simultaneous transmissions and receptions on the same frequency band. For this reason, a multiple access technique called TDD-CDMA is used where mobile terminals in different hops along the routing 
path share the same frequency band but transmit using different spreading codes and use time division duplexing [80]. Time is divided into fixed timeslots equal to the time required to transmit a packet. Terminals are separated into two groups, with every terminal in transmit mode for half of the timeslots and in receive mode for the other half of the timeslots. Therefore a two timeslot system is used. Terminals are grouped in a manner so that no terminal is ever sending traffic to a destination terminal that is also transmitting. The use of the layer number of each mobile terminal is the perfect mechanism to accomplish this grouping of terminals, where the layer number was defined as the number of hops required to reach a base station. Any mobile terminal in an even numbered layer can transmit on all even numbered timeslots, whereas any mobile terminal in an odd numbered layer receives on all even timeslots. In an odd timeslot, all mobile terminals in an odd numbered layer can transmit whereas all mobile terminals in an even numbered layer can only receive. Any terminal that has a packet to transmit will do so on any timeslot that it is in transmit mode. Terminals that are transmitting on the same timeslot will do so using different spreading codes. It is necessary that mobile terminals within a few hops of each other are synchronized so that they do not create large amounts of interference by transmitting on the wrong timeslot. Figure 3.7 shows how terminals that are in different layers transmit on different timeslots in a multihop cellular network. Figure 3.8 shows an example of how packet transmission occurs using TDD in a multihop cellular network.

\subsection{Power Control}

In a CDMA network, the SINR at the receiver should be sufficiently high that the intended transmission can be correctly decoded and at the same time not be too high that too much interference is created. For this reason, receivers use a power control mechanism that adjusts the transmission power of the transmitting terminals so that all signals 


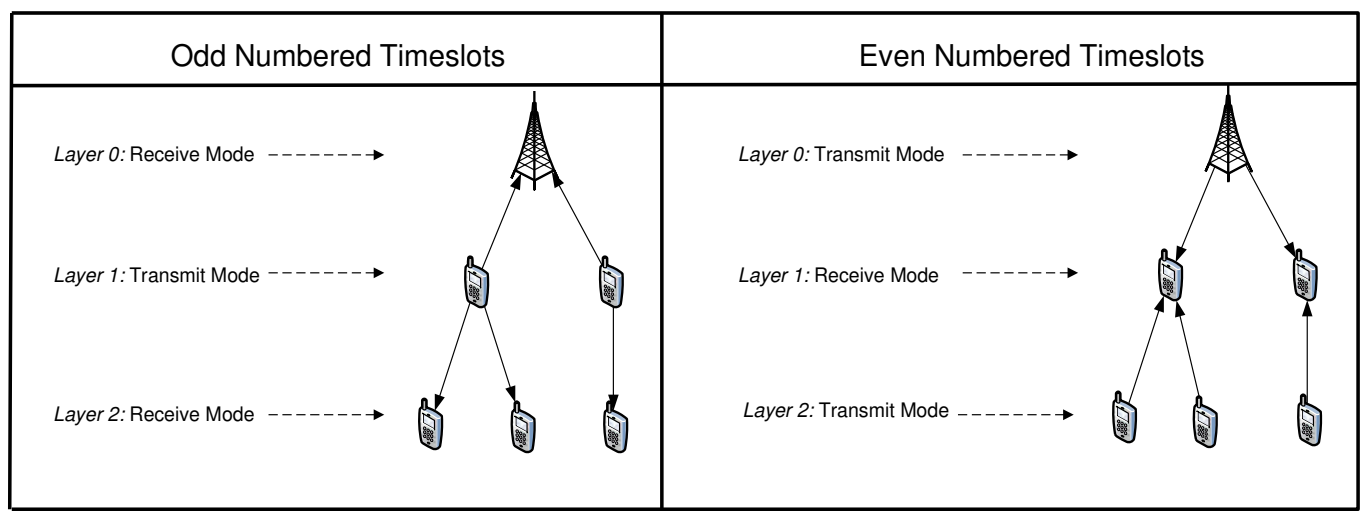

Figure 3.7: Use of Timeslots in a Multihop Cellular Network

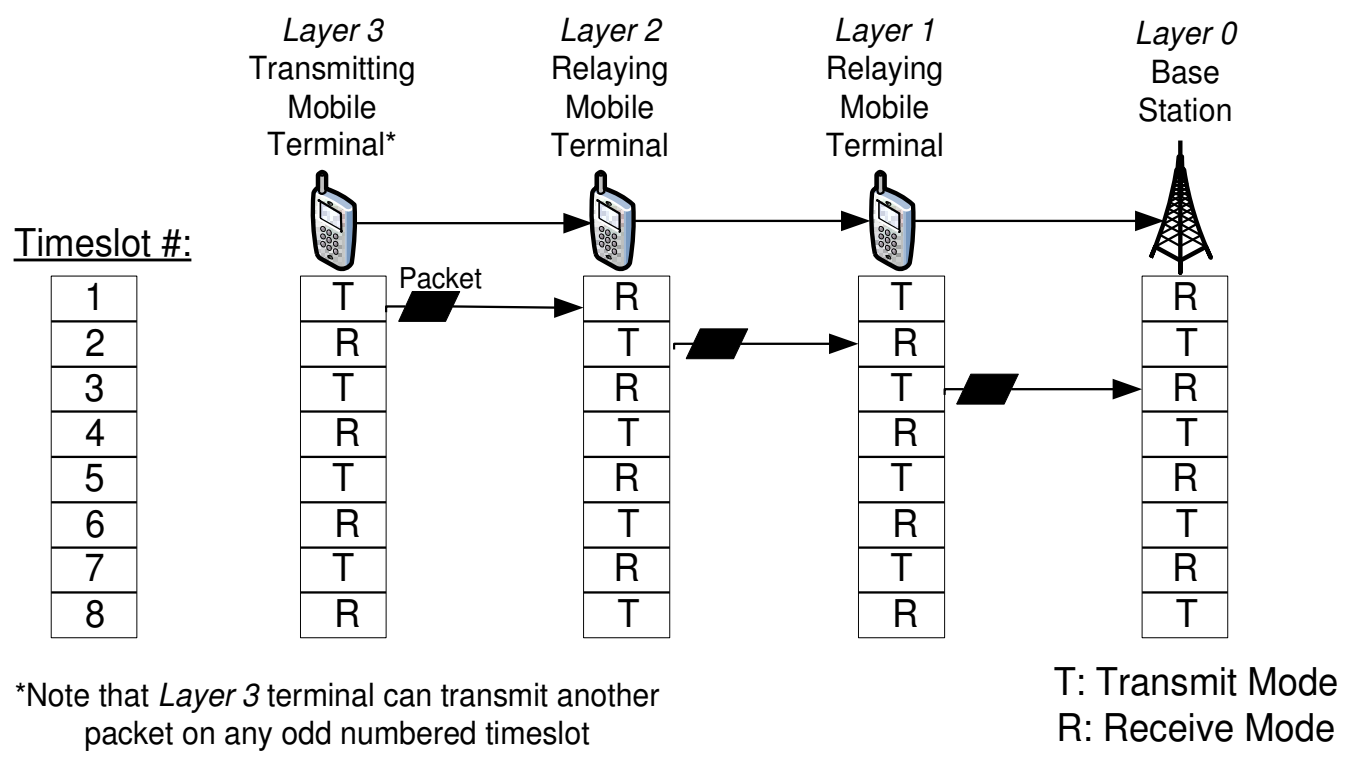

Figure 3.8: Example of Packet Transmission using TDD in a Multihop Cellular Network have equal SINR at their intended receivers.

This power control mechanism can function by sending power control messages in one of two ways. One possible way is to use a separate control channel to transmit power control messages. This would be a good mechanism to use if the mobility of terminals is large because power can be adjusted rapidly using the control channel. An alternative way would be to piggyback the power control information onto all transmissions that are made from the source to the destination. In this scheme, whenever a transmission is made, the transmitted power is adjusted. This would be a good mechanism if the 
mobility of the terminals is small.

Power control in our architecture works in a manner similar to the closed-loop power control system used in cellular networks like W-CDMA as discussed in chapter 2. Power control works similarly in that messages are sent between the source and the destination on the control channels with a command-react cycle of $1500 \mathrm{~Hz}$. Power control occurs using a step size of $1.0 \mathrm{~dB}$ meaning that transmitter power increases by $1.0 \mathrm{~dB}$ if the received power is too low and decreases by $1.0 \mathrm{~dB}$ if the received power is too high. This command-react cycle ensures that the adjustments in transmit power occur faster than any fading mechanism. Power control works differently in a multihop cellular system because it can occur between a relaying mobile terminal and a child terminal instead of the mobile terminal and the base station in the traditional cellular system. Therefore an inverse channel power control method is used and we assume that all packets arrive at their intended destination with an equal amount of received power per bit of information.

\subsection{Simulation Parameters}

Several parameters that are used in simulations throughout the remainder of this thesis are now presented. The majority of the parameters come from the W-CDMA standard, the most widely used $3 \mathrm{G}$ network. A spreading bandwidth $W$ of $5 \mathrm{MHz}$ is used along with a chip rate of $3.84 \mathrm{Mcps}$. The data rate $R$ of mobile terminals is $60 \mathrm{kbits} / \mathrm{s}$. Using QPSK, 30 ksymbols/s can be transmitted leading to a processing gain $N$ of 128 . A maximum bit error rate (BER) of $10^{-3}$ can be supported by our system and therefore for correct reception the SINR must remain above a threshold $\alpha=7 \mathrm{~dB}$ [23]. The path loss exponent $l$ is 4 , the lognormal shadowing coefficients have $\sigma=8 \mathrm{~dB}$. Simulations are averaged upon 500 different realizations of positions of the mobile terminals and each realization simulates 1000 timeslots. Mobile terminals are randomly distributed in the cell with a uniform probability distribution function. Terminals are considered 
stationary to reduce the complexity of simulations. Each mobile terminal will generate Poisson distributed traffic with an arrival rate of $\lambda=0.1$ packets per timeslot. 


\section{Chapter 4}

\section{Effects of Intercell Interference and}

\section{Correlated Shadowing}

In this chapter, the effects of intercell interference and correlated shadowing in a multihop cellular network are investigated. We begin by taking a detailed look at the intercell interference model as well as presenting the correlated lognormal shadowing model used in this chapter. We then present an analysis of the multihop cellular network where the SINR at the base station is derived. Simulation results are then presented that show that intercell interference is effectively eliminated in a multihop cellular network. Our results also show that when intercell interference and correlated shadowing are considered the SINR at the base station is higher and the energy consumption is lower than in a traditional cellular network. This differs from the results of a network consisting of a single cell where the SINR in a multihop cellular network was found to be slightly lower than in a traditional cellular network [10][11].

\subsection{Motivation}

The reverse link capacity of any CDMA cellular network is dependent on the total interference at the base station. The total interference at the base station is comprised of 
both in-cell interference and intercell interference. The majority of the intercell interference at a base station is caused by transmissions of mobile terminals that are near the boundaries of neighboring cells. In a traditional cellular network, the in-cell and intercell interference typically account for two-thirds and one-third of the total interference in a cell, respectively. In multihop cellular networks, the transmission power of terminals near the cell-edge of neighboring cells is lower than in traditional cellular networks which may result in the creation of a lesser amount of intercell interference.

A multihop cellular network architecture was proposed in [10][11] where minimum hop routes were found from every mobile terminal to a base station using a distributed routing algorithm. The advantage of using minimum hop routes is that they minimize interference at the base station while maintaining a low overall energy consumption in the network. However, the models used in these papers and other works prior to [15] only considered one-cell systems and did not take into account the effects of intercell interference on network performance whereas real multihop cellular networks would consist of multiple cells. Moreover, independent lognormal shadowing coefficients from each terminal to the base station were used whereas experimental results show that shadowing coefficients are correlated [20]. In this chapter, the work in [10][11] is extended by incorporating intercell interference and correlated shadowing into our model and by observing their impact on the SINR at the base station and on the overall energy consumption in a multihop cellular network.

\subsection{Intercell Interference Model}

To investigate the effects of intercell interference, we will compare the performance of the multihop cellular network when it is modeled as consisting of multiple cells to when it is modeled as a single cell. As mentioned in section 3.1, our multihop cellular network is modeled using a central reference cell as well as its six neighboring base stations. All cells 
use the same frequency band leading to a frequency reuse factor of 1 . All of our results are obtained from the reference cell with neighboring cells being treated solely as sources of interference as seen in Figure 4.1. Interference emanating from these neighboring cells will reach the reference cell resulting in performance degradation.

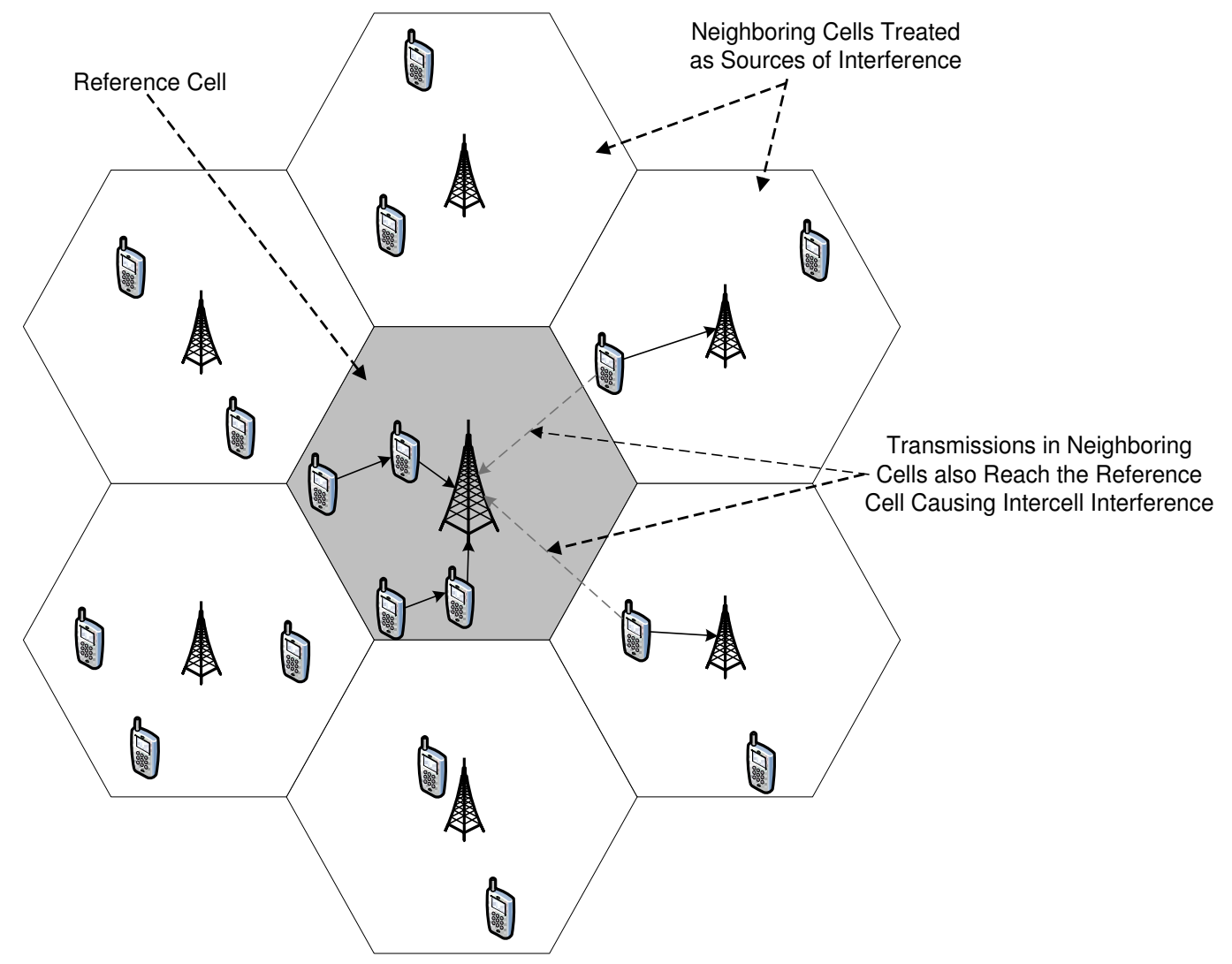

Figure 4.1: Intercell Interference in a Multihop Cellular Network

The intercell interference is modeled in a manner similar to the in-cell interference. The received intercell interference power $P\left(d_{i j}\right)$ at terminal $j$ in the reference cell from terminal $i$ in a neighboring cell transmitting with power $P_{i}$ is

$$
P\left(d_{i j}\right)=P_{i} \cdot d_{i j}^{-l} \cdot 10^{\xi_{(0, \sigma)} / 10}
$$

where $d_{i j}$ is the distance between terminals $i$ and $j, l$ is the path loss exponent and $\xi_{(0, \sigma)}$ is a Gaussian distributed random variable with zero mean and standard deviation $\sigma$. 


\subsection{Correlated Shadowing Model}

To investigate the effects of correlated shadowing, we update the propagation model presented in section 3.2 to incorporate correlated lognormal shadowing coefficients. The correlated shadowing model used is the Angle-of-Arrival Difference(AAD)/Distance dependent correlation model developed in [31], which approximates the experimental results of Sorensen [65]. This model makes use of the fact that terminals that are close to one another and that are transmitting in the same direction have highly correlated shadowing coefficients. As shown in Figure 4.2 mobile terminals having a large common propagation path to a base station have high shadowing correlation and mobile terminals having a small common propagation path to a base station have low shadowing correlation.

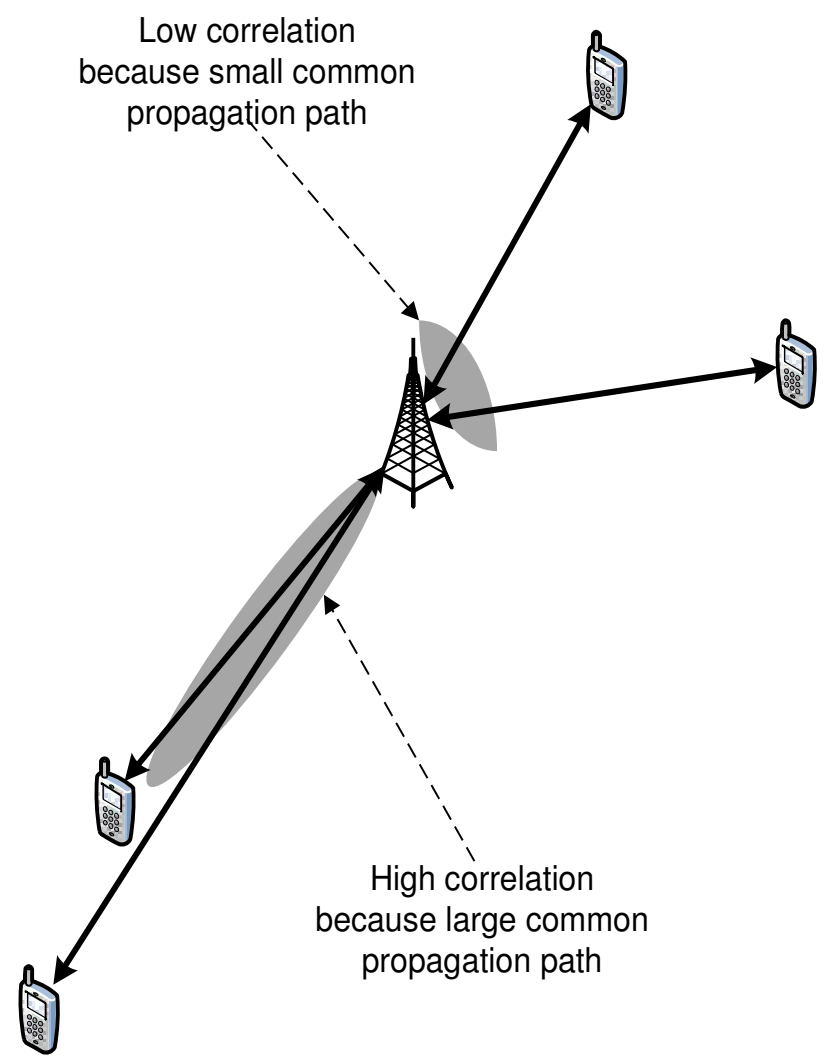

Figure 4.2: Correlation of Shadow Fading

Correlated lognormal shadowing coefficient $\gamma_{i j}$ between terminal $a$ and the base station denoted 0 is 


$$
\gamma_{a 0}=10^{\xi_{(0, \sigma)} / 10}
$$

where $\xi_{(0, \sigma)}$ is a correlated Gaussian distributed random variable with zero mean and standard deviation $\sigma$. Correlated Gaussian random variables are formed using an $M \mathrm{x} M$ correlation matrix $\boldsymbol{\rho}$ with correlations $\rho_{a b}$ for terminals $a=1, \ldots, M$ and $b=1, \ldots, M$ found as follows.

$$
\boldsymbol{\rho}=\left[\begin{array}{cccc}
1 & \rho_{12} & \ldots & \rho_{1 M} \\
\rho_{21} & 1 & \ldots & \rho_{2 M} \\
\vdots & \vdots & \ddots & \vdots \\
\rho_{M 1} & \rho_{M 2} & \ldots & 1
\end{array}\right]
$$

The correlation $\rho_{a b}$ is

$$
\rho_{a b}(\theta, R)= \begin{cases}f(X, R)\left(0.8-\frac{|\theta|}{75}\right) & \text { if }|\theta| \leq 60^{\circ} \\ 0 & \text { if }|\theta|>60^{\circ}\end{cases}
$$

where $\theta$ is the angle formed between two mobile terminals $a, b$ and the base station and $f(X, R)$ is

$$
f(X, R)= \begin{cases}1-\frac{R}{X} & \text { if } R \leq X \mathrm{~dB} \\ 0 & \text { if } R>X \mathrm{~dB}\end{cases}
$$

$\mathrm{X}$ denotes the point where the distance dependent correlation reaches its minimum value, after which the correlation is 0 . From experimental results, $\mathrm{X}$ is found to be approximately $20 \mathrm{~dB}$ [31]. $\mathrm{R}$ is found using

$$
R=\left|10 \log _{10}\left(\frac{\mathrm{d}_{\mathrm{a} 0}}{\mathrm{~d}_{\mathrm{b} 0}}\right)\right|[\mathrm{dB}]
$$

where $d_{a 0}$ and $d_{b 0}$ denote the distance of mobile terminals $a$ and $b$ to the base station. Correlated Gaussian random variables $\xi_{1}, \xi_{2} \ldots \xi_{M}$ can be formed from uncorrelated Gaussian random variables $x_{1}, x_{2} \ldots x_{3}$ as follows. 


$$
\xi=\mathrm{CX}
$$

where $\boldsymbol{\xi}$ is a vector containing the correlated Gaussian random variables, $\mathbf{X}$ is a vector containing uncorrelated Gaussian random variables and $\mathbf{C}$ is a matrix of weight coefficients. The weights in $\mathbf{C}$ must be determined such that each pair of correlated variables $\left\{\xi_{a}, \xi_{b}\right\}$ have correlation $\rho_{a b}$. Furthermore it is required that the standard deviation $\sigma_{\xi}=\sigma_{x}$.

The correlation matrix $\boldsymbol{\rho}$ can be decomposed as follows with weight matrix $\mathbf{C}$ found using the Cholesky factorization [19].

$$
\rho=\mathrm{CC}^{\mathrm{T}}
$$

In order for a solution of the Cholesky factorization to exist, $\boldsymbol{\rho}$ must be positive semidefinite resulting in a matrix $\mathbf{C}$ that is lower triangular. Using equation 4.7 the correlated Gaussian random variables can be computed. The Cholesky factorization ensures that $\sigma_{\xi}=\sigma_{x}$. Then using equation 4.2 the correlated lognormal shadowing coefficients can be found [31].

\subsection{Capacity}

One of the main objectives when designing a multihop cellular network is to maximize system capacity. To have a high capacity, the network must support a large number of high bit rate users at a sufficient SINR $>\alpha$ to obtain a required BER. To achieve this, the SINR of each transmitting terminal should be equal at the receiver which is accomplished using power control.

The bottleneck of a multihop cellular network is the base station because it is the location where the lowest SINR occurs as all traffic must eventually pass through it. For this reason, the analysis of the SINR at the base station is now presented. Due to the 
careful design of the routing paths in the minimum hop routing algorithm, it can be noted that the majority of the interference at the base station is due to the transmissions of its children. Each of its children's transmissions have equal SINR at the base station due to the use of power control. Note that transmissions from terminals in even layers do not interfere with transmissions from terminals in odd numbered layers thanks to the TDDCDMA mechanism involving the use of two distinct timeslots. Transmitting terminals that are more than two hops away from the base station create minimal interference there because those terminals are not even within range of any terminal in layer 1 that transmits directly to the base station.

The probability of bit error in a multihop cellular CDMA system is a monotonically decreasing function of the SINR:

$$
\begin{gathered}
P_{\text {error }}=f\left(\operatorname{SINR}_{\mathrm{MCN}}\right) \\
P_{\text {error }}=f\left(\frac{E_{b}}{N_{0}+I}\right)
\end{gathered}
$$

Denote the total number of transmitting terminals in layer $i$ as $M_{i}$. When the base station has $C$ children transmitting to it.

$$
P_{\text {error }}=f\left(\frac{E_{b}}{N_{0}+\frac{\Psi}{N} \sum_{j=1}^{C-1} E_{b}+\frac{\Psi}{N} \sum_{k=1}^{M_{3}} E_{b, k}}\right)
$$

where $E_{b}$ is the energy received from the children of the mobile terminal and $E_{b, k}$ is the energy received from the $k^{\text {th }}$ transmitting terminal in layer 3 .

Note that $E_{b, k}$ is much smaller than $E_{b}$ because terminals that are more than two hops away from the base station create a negligible amount of interference there. If we assume that $N_{0}$ is small and that the interference at the base station generated by terminals in 
layer 3 is small compared to the interference created by the children of the base station $E_{b, k}<<E_{b}$,

$$
\operatorname{SINR}_{\mathrm{MCN}} \approx \frac{E_{b}}{\frac{\Psi}{N}(C-1) E_{b}}
$$

From this we can find the total number of transmitting children $C$ that a receiver can support while maintaining an $\operatorname{SINR}>\alpha$.

$$
\begin{aligned}
& \alpha \leq \frac{N}{\Psi(C-1)} \\
& C \leq \frac{N}{\Psi \cdot \alpha}+1
\end{aligned}
$$

This gives us the maximum number of children that can be transmitting a single packet to the base station while still maintaining a sufficient $\operatorname{SINR}>\alpha$. This is similar to the result obtained for a traditional cellular CDMA network but instead of being the total number of mobile terminals transmitting in a cell, this equation gives us the total number of transmitting children that a base station can have. However this equation does not consider that a terminal may have to transmit multiple packets to the base station. This occurs when a mobile terminal generates its own traffic and in addition is required to relay traffic for another terminal or if it is required to relay packets for multiple terminals. Mobile terminals that forward packets for other terminals have to transmit at a higher rate and this must be taken into consideration. $\beta$ is defined as the total number of packets being transmitted to the base station at a given timeslot. Effectively a terminal that has to forward one packet and transmit one packet of its own acts like a user transmitting at twice the typical bit rate as is done in a W-CDMA network. The total number of packets that can be transmitted to the base station at a given time is: 


$$
\beta \leq \frac{N}{\Psi \cdot \alpha}+1
$$

This formula only takes into account a network consisting of a single cell. When a network with many cells is considered, interference from transmissions in other cells arriving at the base station decreases the maximum number of packets $\beta$ that can be correctly received. This can be modeled by multiplying $\beta$ by a factor $F \leq 1$. In a traditional cellular network, the value of $F$ was approximately equal to $\frac{2}{3}$. The amount of intercell interference at the base station in a multihop cellular network is drastically reduced because transmission ranges are much smaller than in a traditional cellular network. Therefore $F$ will approach 1 in a multihop cellular network as the maximum transmission range of each terminal is decreased as will be shown in section 4.5. Taking this into account we can update our capacity equation, so that the maximum number of packets that can be transmitted to the base station in a given timeslot is:

$$
\beta \leq \frac{N \cdot F}{\Psi \cdot \alpha}+1
$$

\subsection{Simulation Results}

In a multihop cellular network, the transmission range of each mobile terminal is decreased as compared to a traditional cellular network. The maximum transmission range in the multihop cellular network is reduced to $\frac{1}{K}$ of the maximum transmission range in a traditional single hop cellular network. Therefore, a value of $K=1$ is equivalent to a traditional cellular network and as we increase $K$, we increase the number of hops that are required for traffic from a given mobile terminal to reach a base station.

Figure 4.3 shows simulations of the average power of the intercell interference at a base station in a multihop cellular network with $M=200$ mobile terminals. The model used for the simulations was presented in chapters 3 and 4 , and the simulation parameters 
are taken from section 3.8 .

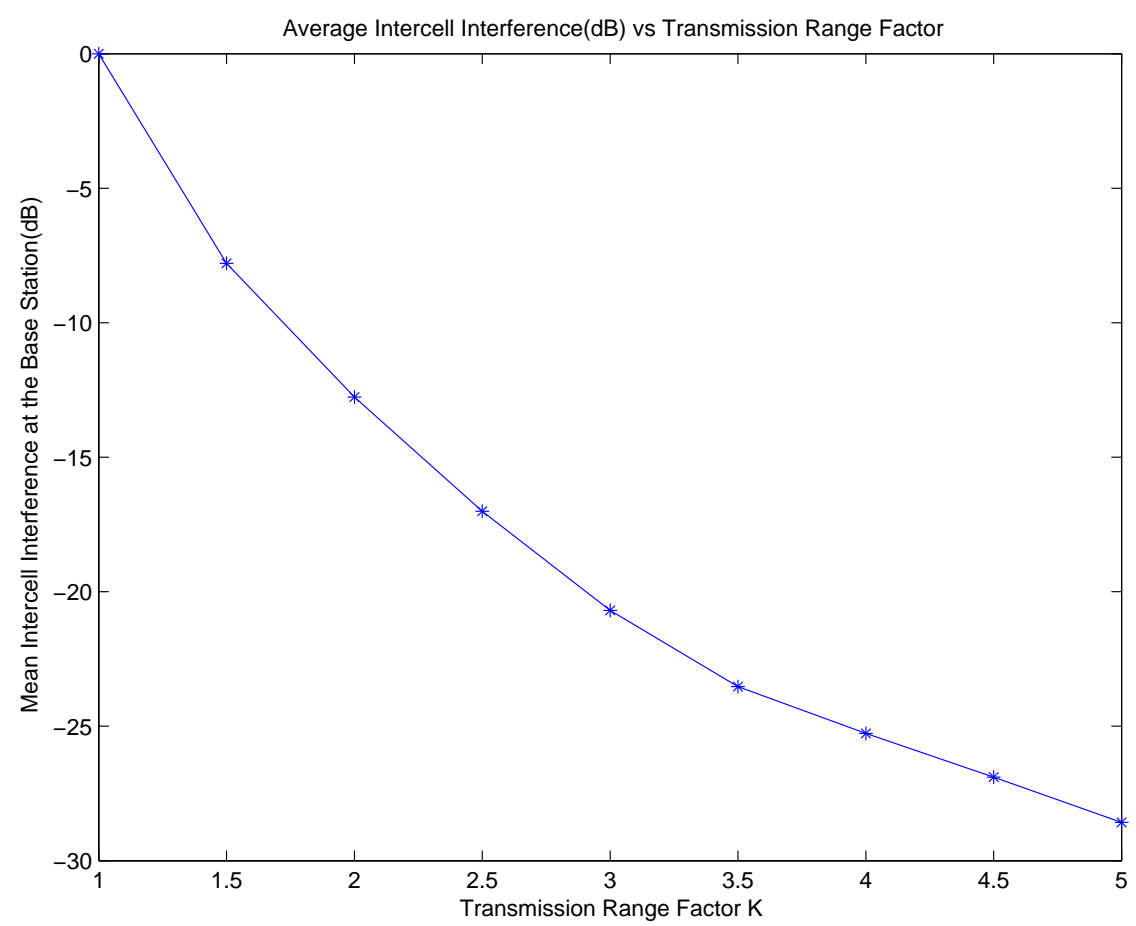

Figure 4.3: Average Intercell Interference vs Transmission Range Factor

Intercell interference has a detrimental effect on the SINR of any CDMA cellular network. From this figure, we can see that the intercell interference is much larger in a traditional cellular network $(K=1)$ than in a multihop cellular network $(K>$ 1). In fact, the intercell interference in a multihop cellular network is up to $28.7 \mathrm{~dB}$ smaller than a traditional cellular network when $K=5$. The reason that the intercell interference of a traditional cellular network is larger is that the transmission power of mobile terminals at the cell edge of neighboring cells is high which creates large amounts of intercell interference. However, in a multihop cellular network, mobile terminals in neighboring cells are able to lower their transmission power because they can use other mobile terminals as relays to forward their traffic to a base station leading to a decrease in intercell interference.

Figure 4.4 shows the average SINR at the base station when simulating the multihop cellular network with correlated lognormal shadowing and multiple cells where intercell 
interference is an issue, as well as in a one-cell network where intercell interference and correlated shadowing are not considered.

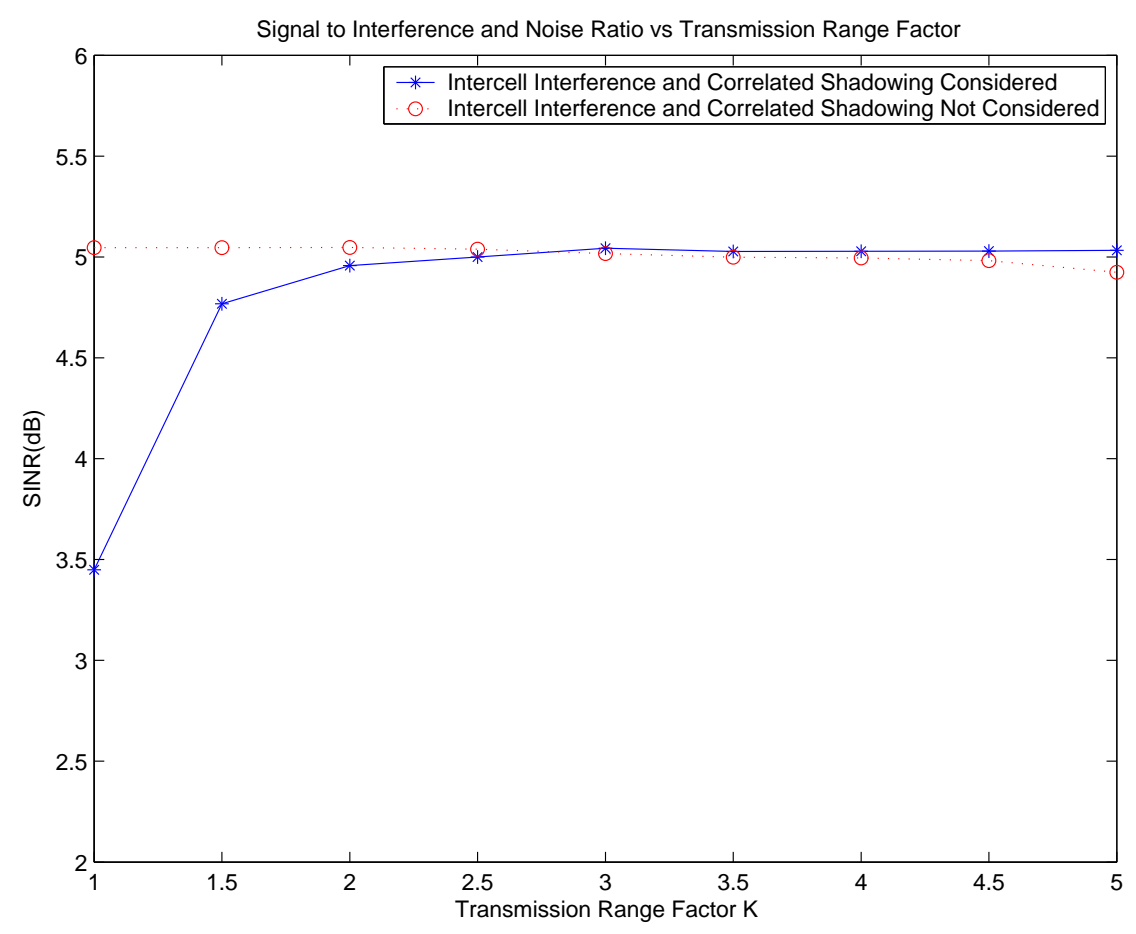

Figure 4.4: Average SINR vs Transmission Range Factor

In a network consisting of a single cell, the SINR of a multihop cellular network ( $K$ $>1)$ is approximately $0.3 \mathrm{~dB}$ smaller than that of a traditional cellular network $(K=$ 1). However when intercell interference is considered, the SINR of a multihop cellular network is approximately $1.6 \mathrm{~dB}$ larger than that of a traditional cellular network. This is due to the near-elimination of intercell interference in a multihop cellular network as seen in Figure 4.3. Therefore, when incorporating intercell interference, not only is the performance in terms of power consumption better than that of a traditional cellular network, but performance in terms of SINR is as well.

Figure 4.5 shows the average power consumption of all terminals in a multihop cellular network when intercell interference and correlated shadowing are considered, as well as when intercell interference and correlated shadowing are not considered.

From Figure 4.5 we observe that the total power consumption in a multihop cellular 


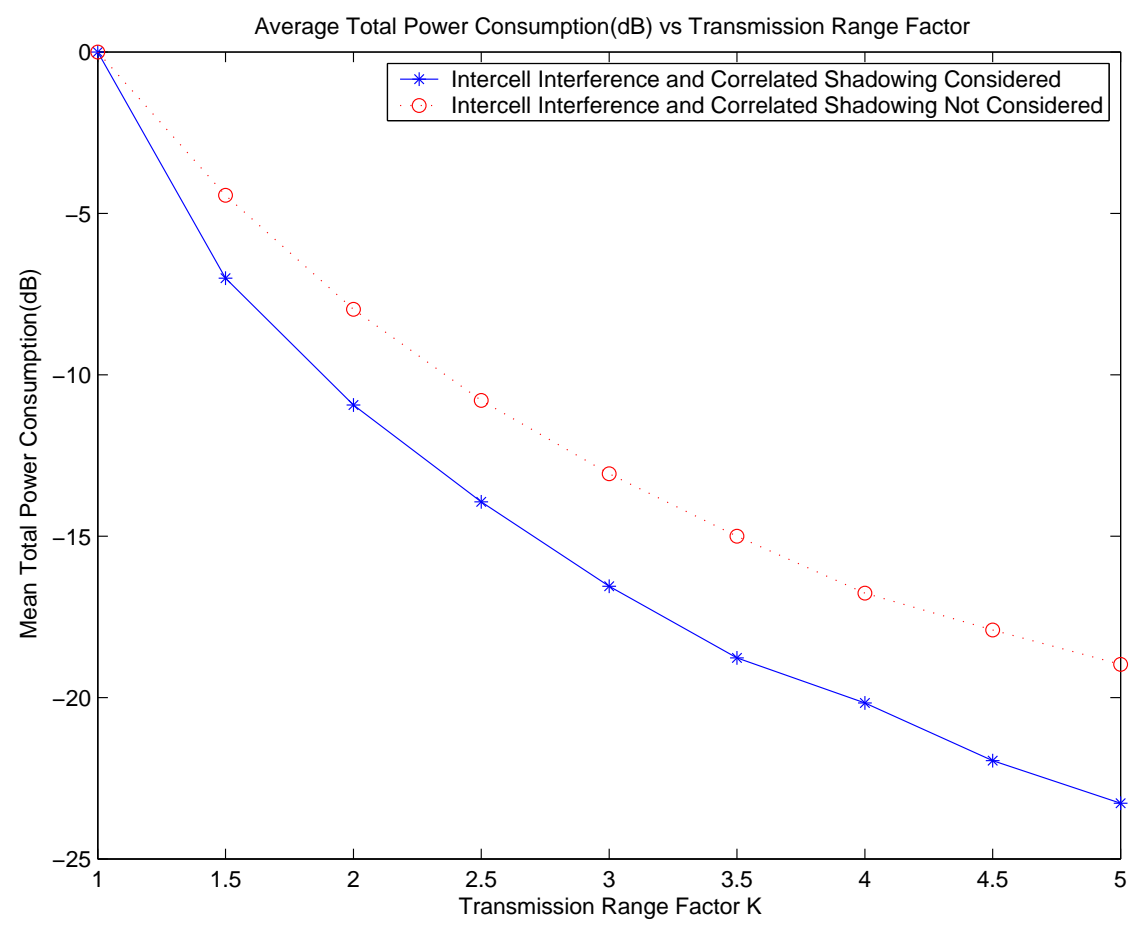

Figure 4.5: Total Power Consumption vs Transmission Range Factor

network is up to $23 \mathrm{~dB}$ lower than in a traditional cellular network. Furthermore, when intercell interference and correlated shadowing are considered, the total power consumption in a multihop cellular network is up to an additional $5 \mathrm{~dB}$ lower than when those factors are not considered. This result is as expected because in a multiple cell traditional cellular network, mobile terminals have to increase their transmission power to account for excess intercell interference to provide a sufficient SINR. However, in a multiple cell multihop cellular network, mobile terminals do not create a large amount of excess interference, and therefore an even greater savings in power consumption is achieved when intercell interference is considered.

\subsection{Chapter Summary}

In this chapter, we considered how intercell interference and correlated shadowing affect the SINR and power consumption of a multihop cellular network. We showed through 
simulation that the intercell interference in a multihop cellular network can be decreased by up to $28.7 \mathrm{~dB}$ as compared to a traditional cellular network. This result implies that the intercell interference which accounts for approximately one-third of the total interference in a traditional cellular network is essentially eliminated in a multihop cellular network.

We also showed that in a multihop cellular network, the SINR at the base station is larger than that of a traditional cellular network by $1.6 \mathrm{~dB}$. An increase of $1.6 \mathrm{~dB}$ means that the multihop cellular network has an SINR that is $44 \%$ higher than that of a traditional cellular network due to the near elimination of intercell interference. The total average power consumption of mobile terminals in a multihop cellular network can also be decreased by up to $23 \mathrm{~dB}$ as compared to a traditional cellular network.

Furthermore, we showed that in a network with many cells, the SINR of the multihop cellular network is higher than the SINR of the traditional cellular network as opposed to being slightly lower when only one cell is considered. When intercell interference is not considered, the multihop cellular network has an SINR that is $0.3 \mathrm{~dB}$ lower than the traditional cellular network whereas when intercell interference is considered the multihop cellular network has an SINR that is higher by $1.6 \mathrm{~dB}$. The performance in terms of overall power consumption is also improved from an $18 \mathrm{~dB}$ power savings to a $23 \mathrm{~dB}$ power savings when intercell interference and correlated shadowing are considered. 


\section{Chapter 5}

\section{Autonomous Infrastructure}

\section{Multihop Cellular Networks}

In this chapter we propose a new multihop cellular network architecture called the autonomous infrastructure multihop cellular network. We begin by presenting several factors that motivate the development of this architecture. The autonomous infrastructure multihop cellular network architecture is then presented along with the interfaces that mobile terminals can use to connect to the backbone network as well as the access point criteria. We then update the system model presented in chapter 3 to incorporate the additional functionality of autonomous infrastructure. We also discuss how access points can be integrated into the minimum hop routing algorithm and the TDD-CDMA mechanism. We then present some analysis that shows that the SINR at the base station in an autonomous infrastructure multihop cellular network is higher than in a traditional cellular network. We also discuss several modes of operation that are possible in an autonomous infrastructure multihop cellular network. We then present simulation results that also show that our proposed architecture leads to an increase in SINR at the base station, an increase in coverage and a lower overall power consumption as compared to a regular multihop cellular network. 


\subsection{Motivation}

A problem that exists in multihop cellular networks that has yet to be addressed is that the base station and the mobile terminals surrounding it are responsible for handling a large amount of traffic. This is because traffic concentration is higher near the base stations on both the forward and the reverse links due to relaying by intermediate terminals. Mobile terminals employ relays for transmissions and therefore the required transmit power of each terminal is reduced as compared to a traditional cellular network. However, this reduction in transmit power does not decrease the amount of in-cell interference in a multihop cellular network. This is because all traffic still emanates or is destined to the base station causing a large congestion of interference near the base station. Therefore the base station is still the capacity bottleneck of the network [41][37][80]. Figure 5.1 illustrates the fact that the base station is the bottleneck of the system.

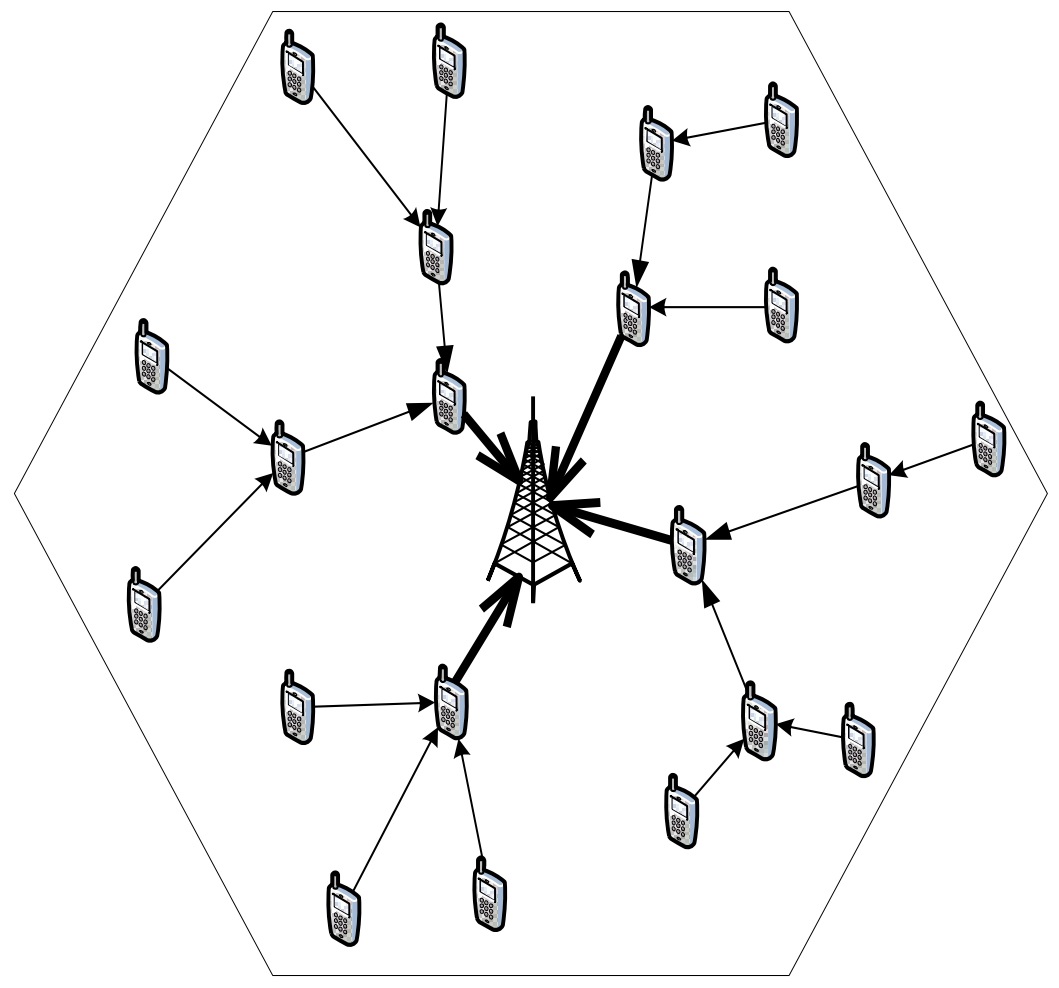

Figure 5.1: Large Amounts of Interference at the Base Station

Typically, when this congestion becomes large, a new base station would be deployed 
to help handle some of the traffic. However, the cost of construction of additional base stations is very high. In this chapter, we present an alternate solution that is costeffective, flexible and scalable to help alleviate the amount of traffic that base stations are required to handle.

\subsection{Autonomous Infrastructure}

We propose a novel multihop cellular network architecture where users can connect their mobile terminals to the backbone network to act as access points and forward the traffic of other terminals directly onto the backbone. We call this proposed architecture the autonomous infrastructure multihop cellular network. This thesis extends our previous work on autonomous infrastructure in traditional cellular networks where access points can be deployed by the user and modifies the concept for it to be applied to multihop cellular networks [66]. In this architecture, we assume that a subset of the mobile terminals in the network are built with an additional interface giving them the potential to transmit directly onto the backbone network. In the future, the cost of manufacturing these mobile terminals may become sufficiently inexpensive that a large percentage of terminals could have this capability. Users can connect their mobile terminal to the backbone network using this interface to improve capacity, coverage and data rates in a certain area. We refer to a mobile terminal as an access point when the terminal is using this interface to establish a direct connection to the backbone network and is able to handle traffic for other users, as would a base station. Access points can connect to the backbone network using a broadband wireless access network, a LAN, a DSL connection, a CATV network or a PLC connection. Depending on their locations, other terminals will be able to forward their traffic to a base station or to any access point that will then send the traffic directly onto the backbone network. This will lower the load on base stations and allow further links to the backbone network to be provided autonomously 
by the user. Allowing mobile terminals in a multihop cellular network to act as access points does not require any major modifications to the physical layer of mobile terminals, apart from the requirement of the additional interface, because terminals already have the capability to transmit directly to one another in a multihop cellular network. For this reason, the multihop cellular network architecture lends itself perfectly to having autonomous infrastructure with certain mobile terminals acting as access points. This is not the case in traditional cellular networks because mobile terminals only have the ability to transmit to base stations and cannot transmit directly to other terminals.

\subsubsection{Backbone Network Connections}

Mobile terminals are built with an additional interface allowing the user to connect his terminal directly to the backbone network giving it the capability to perform the functions of an access point. Connecting to the backbone network should be very simple in nature resulting in a 'plug-and-play access point'. In fact, the connection to the backbone network may even be wireless resulting in a 'wireless access point'. The various types of interfaces that a mobile terminal could use to connect to the backbone network are now detailed [66]:

1. Wireless Access Network: A user could connect his terminal to the backbone network using an interface to a broadband wireless access network. A primary example of this would be a WiFi connection to a wireless LAN using the IEEE 802.11 protocol. Mobile terminals are already increasingly being constructed with this type of additional interface and this trend is expected to continue. Users could configure their terminal to require them to manually connect to the wireless access network or they could allow their terminal to automatically connect to any wireless network that is within range.

2. Local Area Network (LAN): A user could connect his terminal to the backbone 
network using an interface to a hardwired Ethernet LAN. Terminals with this interface could plug directly into the LAN where they would auto-configure an IP address to connect to the backbone network. At this time the terminal would acquire the IP address of the network controller and initiate communication with it. The mobile terminal is now called an access point as it can provide a similar functionality as the base station. Subsequently the access point would autonomously configure its transmission parameters to optimal values as discussed in chapter 6 to allow it to handle traffic for other users.

3. Public Switched Telephone Network (PSTN): A user could connect his terminal to the backbone network using an interface to the PSTN. An example of this would be a Digital Subscriber Line (DSL) connection that could use one of several DSL technologies such as ADSL, VDSL, etc [5]. Terminals with this interface could be plugged into the backbone network as easily as an existing DSL modem.

4. Community Access Television (CATV) Network: A user could connect his terminal to the backbone network using an interface to the CATV network. Having an interface that connects to the CATV network is reasonable as the CATV network has a high capacity and is widely deployed in residential areas [55]. Terminals with this interface could be plugged into the backbone network as easily as a typical cable modem.

5. Power Line Communications (PLC) Network: A user could connect his terminal to the backbone network using an interface to a PLC network. When PLC is used to provide broadband access to the Internet through regular power lines, it is typically referred to as Broadband over Power Lines (BPL) or power-line Internet [47]. A mobile terminal would only need to plug its BPL interface into any outlet in a BPL equipped building to connect to the backbone network. The advantage of BPL over CATV and DSL connections is that power lines already have ubiquitous 
availability even in remote locations where CATV and DSL connections may not exist. The disadvantage of BPL is that power lines are inherently a very noisy environment which limits the amount of bandwidth and Quality of Service (QoS) that BPL can provide as compared to CATV or DSL networks.

\subsubsection{Access Point Criteria}

A mobile terminal has to meet certain criteria in order to become an access point. Firstly, it has to be connected to the backbone network. Secondly, a terminal must have significant transmit and processing power capabilities as well as battery power to handle the burden of being an access point and handling the traffic of many users. Thirdly, a terminal chosen to be an access point should be stationary when it is connected to a LAN or semi-stationary when it is connected to a wireless access network so that it does not leave the transmission range of the wireless network or of the other mobile terminals that it is serving. For example, a user walking around his home with his mobile terminal would be considered stationary enough to become an access point if the entire home is within coverage of a Wireless Local Area Network (WLAN). Having as many portals to the backbone network as possible is preferable in terms of reducing interference surrounding the base station. Therefore, any mobile terminal that satisfies these criteria will perform the functionality of an access point.

\subsubsection{Access Point Functionality}

Access points do not disrupt regular base stations because an access point acts very similarly to an additional base station with lesser capability. Therefore access points and regular base stations are interoperable. Whereas a base station is able to handle traffic from a large number of users, an access point is only able to handle traffic from a small number of users. The capability of an access point depends on the processing and transmit power capabilities of the mobile terminal as well as the bandwidth of its link 
to the backbone network. The autonomous infrastructure multihop cellular network will therefore consist of cells with varying numbers of terminals and access points, and its structure will differ significantly from that of a traditional cellular network or a regular multihop cellular network. In this type of network, service providers are not required to make further investments towards infrastructure deployment in heavily built up areas and further links to the wireline network backbone are provided autonomously by the user at his own cost. This proposed network is flexible, scalable and very robust at a lower cost than a conventional infrastructure-based network.

\subsubsection{Other Design Issues}

Every access point can connect to a centralized network domain controller that assists access points in issues involving their configuration, security and billing. Access points connect to the network domain controller using their link to the backbone network. The network domain controller is in charge of providing many important access services and security functions. Examples include the authentication of a user and the establishment of a call. The network domain controller is also responsible for the distribution of CDMA spreading codes to the access points and the mobile terminals that it is serving. When a user wants to make a transmission, the network domain controller will set up a secured session between the terminal and the access point, and is in charge of the encryption of transmissions if necessary. This encryption may be achieved with a point-to-point encryption of the transmission on the wireless link. However, transmissions may have to be relayed by multiple mobile terminals to reach their destination and therefore an end-to-end tunneling protocol may be more appropriate so that intermediate relaying terminals cannot view sensitive information that they do not have permission to access.

The network domain controller is also responsible for issues involving billing for the usage of the wireless network. Through the access point, the network domain controller can find the amount of airtime used or the amount of data transmitted by a mobile 
terminal. The charges for the wireless access can then be issued to the mobile user as either a charge for the amount of time used or the amount of data sent. Alternatively, a flat-rate service may be offered by the network operator. Furthermore, some type of credit should be issued to the mobile terminal that is being used as an access point. This would provide incentive to the terminals to act as access points and stimulate cooperation among terminals in the network. For example, any terminal could have free access to the wireless network while it is serving as an access point. Alternatively, a terminal could be issued a number of credits that would entitle it to a certain amount of free access to the network in the future. This genre of incentive scheme can be extended to reward other terminals that are in charge of relaying a large amount of traffic. This issue has already been addressed for regular multihop cellular networks in [61][28]. A simple modification of this scheme can be made so that incentives are given to access points in addition to relaying mobile terminals as a reward for handling traffic for other users.

The autonomous optimization of transmission parameters of access points is a major design issue for autonomous infrastructure multihop cellular networks. The optimization of the transmission ranges of mobile terminal is an extremely important issue because the choice of transmission range has a large impact on most network performance metrics including SINR, coverage and power consumption. This problem is dealt with in detail in chapter 6.

\subsection{Updated Network Model}

The proposed autonomous infrastructure multihop cellular network is analyzed using the following model. The system model is very similar to the one presented in chapter 3 and therefore in this section we only present the modifications that are made to that model to incorporate autonomous infrastructure. Aspects of the system model that were presented in chapter 3 such as the minimum hop routing algorithm, the spreading codes, the power 
control mechanism and the TDD-CDMA mechanism are also used in the autonomous infrastructure multihop cellular network; the only difference being that they are used by access points in addition to base stations.

In the autonomous infrastructure multihop cellular network the coverage area is broken up into smaller coverage areas that are approximated using hexagonal cells. A base station is located at the center of each cell and each base station has 6 neighboring base stations. Results are obtained from the central reference cell with the 6 neighboring cells being treated solely as sources of interference as seen in Figure 5.2. A number of mobile terminals $M$ is uniformly distributed in each cell of radius $R$. All mobile terminals that are connected to the backbone network and meet the previously discussed criteria act as access points. We assume that each mobile terminal has an equal probability of being connected to the backbone network. We denote $p_{a p}$ as the probability that a mobile terminal is connected to the backbone network and can act as an access point. The average number of access points in a cell is therefore $M p_{a p}$. The base station and all access points are in charge of forwarding traffic from other mobile terminals onto the backbone network. The transmission range $r$ of base stations, access points and mobile terminals is lower than their range in a traditional cellular network $R$ by a factor of $K$ which is a parameter called the transmission range factor and $r=\frac{R}{K}$. Transmissions of traffic towards access points are assumed to occur on the same frequency band as transmissions of traffic towards base stations. Minimum hop routing paths are used to connect the mobile terminals to the base station and the access points as will be described in section 5.4.1. TDD-CDMA is also used for traffic being relayed to access points as will be described in section 5.4.2. 


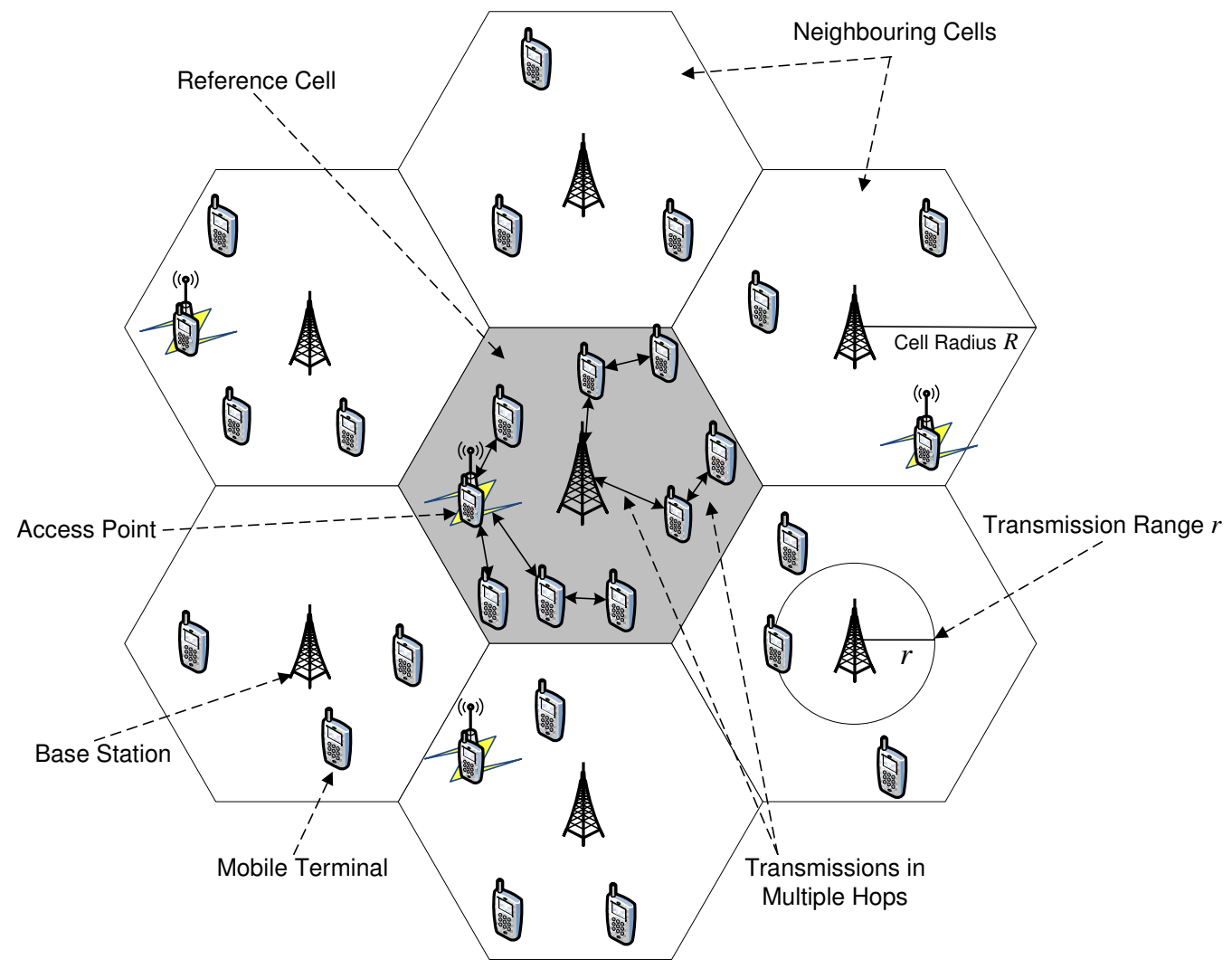

Figure 5.2: Autonomous Infrastructure Multihop Cellular Network

\subsection{Autonomous Infrastructure Multihop Cellular Net- work Design}

The autonomous infrastructure multihop cellular network should be designed in a manner that the interference at the base station and the interference at the access points are minimized. This is due to the fact that the capacity of CDMA networks is interference limited [36]. The interference at the base station should be minimized because it is the network capacity bottleneck and reducing interference there will allow it to support more users at higher bit rates. The interference at the access points should be minimized to allow them to handle traffic for a larger number of users so that less traffic is required to be handled by the base station, thus reducing the interference at the bottleneck. Minimum hop routing was found to minimize interference at the base station in a regular multihop 
cellular network [10][11]. In section 5.4.1 we show how access points can be integrated into the minimum hop routing algorithm. Transmissions between mobile terminals and access points should be made in a manner such that large amounts of interference do not reach the base station while it is receiving. TDD-CDMA is an efficient method of ensuring that large amounts of interference are not created between transmitting and receiving terminals on different hops along the routing path. In section 5.4.2 we show how access points can be integrated into the TDD-CDMA mechanism.

Recall that a terminal that transmits to a base station using $x$ hops is said to be part of the base station's $x^{\text {th }}$ layer. Similar terminology will be used for access points. A terminal that forwards its traffic towards an access point using $x$ hops will be said to be part of the access point's $x^{\text {th }}$ layer. Although access points do not forward traffic towards the base station, we say that an access point would be part of a base station's $y^{\text {th }}$ layer if it would require $y$ hops to reach the base station had it been a regular mobile terminal.

\subsubsection{Integration of Access Points into Minimum Hop Routing Algorithm}

Access points use the minimum hop routing algorithm to find routing paths to mobile terminals so that access points can handle the traffic of some terminals. A full description of the minimum hop routing algorithm is presented in the system model in section 3.5. Some clarifications as to how access points can be integrated into the algorithm are given here. The minimum hop routing algorithm for access points works in a similar manner as for base stations. The main difference is that routing paths to a base station are already created when an access point is activated. When a user connects a mobile terminal to the backbone network and it has the capability to act as an access point, a route discovery process is initiated. The route discovery process begins at the access point where it transmits its pilot signal with a transmission range $r=\frac{R}{K}$. Optimal values of the transmission range factor $K$ are found autonomously by the access point as 
described in chapter 6 . Any mobile terminal that correctly receives this pilot signal and has not received a higher strength pilot signal from another access point or base station will transmit directly to that access point. Those mobile terminals will be said to belong to that access point's layer 1 because they are within one hop of that access point.

Each mobile terminal in the access point's layer 1 will then transmit a pilot signal to all mobile terminals within its range. Every terminal that receives multiple pilot signals chooses to relay traffic to the terminal with the lowest layer number. For example, any terminal that receives a pilot signal from a terminal in layer 1 will not route its traffic to that terminal if it also receives a pilot signal from a base station. This ensures that minimum hop routes are preserved. When a terminal receives pilot signals from multiple terminals with the same layer number it will route its traffic to the terminal with the highest received pilot power. Therefore terminals that are not in a layer 1 that receive a pilot signal from an access point's layer 1 terminal will choose to route their traffic to that terminal if it has the highest received pilot power of all layer 1 terminals. These terminals become part of the access point's layer 2. This process will repeat for higher layered terminals. Note that this process is halted if the maximum number of terminals that can be supported by an access point has been reached.

\subsubsection{Integration of Access Points into TDD-CDMA Mecha- nism}

In order that traffic being transmitted to access points does not create large amounts of interference for traffic being transmitted to base stations, traffic being handled by access points will also use TDD-CDMA but the allocation of timeslots will differ from those for base stations. For traffic being handled by base stations, even layered terminals transmit on even numbered timeslots and receive on odd numbered timeslots and odd layered terminals transmit on odd numbered timeslots and receive on even numbered timeslots as described in section 3.6. However, this scheme can not be used for access 
points because it would result in large amounts of interference being created when a terminal that would normally be transmitting on an odd numbered timeslot becomes an access point. This would create the situation where many terminals transmitting towards an access point are close to relaying terminals that are receiving traffic destined to a base station. This would create large amounts of interference because a terminal that is transmitting on a given frequency should not be close to a terminal that is receiving on that frequency unless the intended destination of the transmitter is the receiver. The negative implications of allocating timeslots in this manner are illustrated in Figure 5.3 which shows an autonomous infrastructure multihop cellular network during an odd numbered timeslot. In this figure, large amounts of interference are created at terminal B because terminals $\mathrm{A}$ and $\mathrm{B}$ are close to one another and terminal A transmits towards an access point at the same time that terminal B is receiving its own traffic. Furthermore, the access point receives traffic at the same time as the base station causing a larger amount of interference at the bottleneck than if no traffic was being transmitted to the access point during that time.
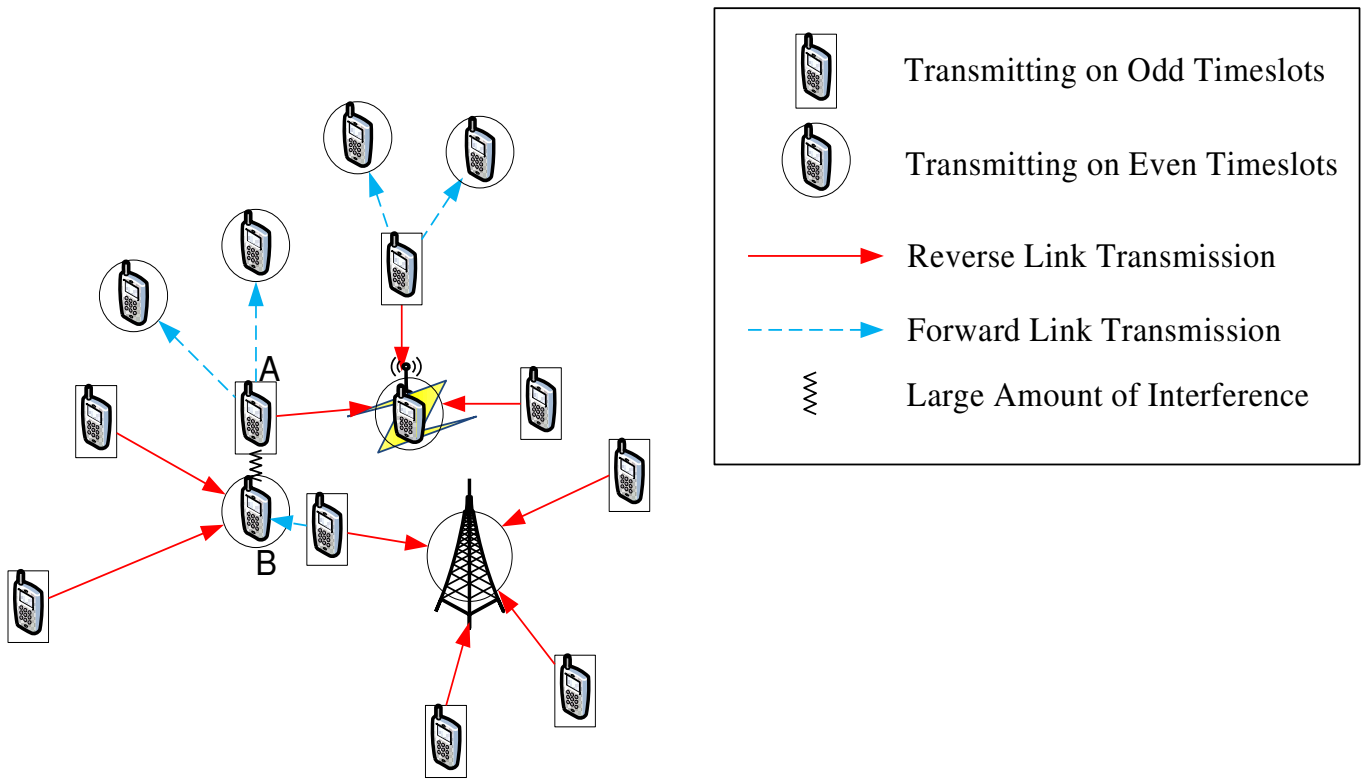

Figure 5.3: Bad Timeslot Allocation for Terminals Transmitting to Access Points 
For this reason, the autonomous infrastructure multihop cellular network is designed so that access points continue transmitting on the same timeslot that they would normally transmit on if they were not access points. Therefore if an access point is an even number of hops away from a base station it will transmit on even numbered timeslots and if it is an odd number of hops away from a base station it will transmit on odd numbered timeslots. Terminals forming the access point's layer 1 will receive on the timeslots where the access point is transmitting and transmit on the alternate timeslots. Terminals forming the access point's layer 2 will transmit and receive on the same timeslot as the access point and so on. Figure 5.4 shows the autonomous infrastructure multihop cellular network during an odd numbered timeslot while displaying the routing paths that are created and how access point transmissions are integrated into the transmission in timeslot TDDCDMA mechanism. Note that for even numbered timeslots this figure is the same except that the arrows would point in the opposite direction and blue arrows would be red and vice versa. In this figure we can see that transmitting and receiving terminals are adequately separated. Furthermore, the access points that are within transmission range of the base station do not receive any traffic while the base station is receiving, thus reducing interference at the bottleneck.

\subsection{Capacity of the Autonomous Infrastructure Mul- tihop Cellular Network}

One of the primary objectives of altering the architecture of the multihop cellular network to include autonomous infrastructure is to increase network capacity. To obtain a high capacity, we need to support a large number of high bit rate users at a sufficient SINR $>\alpha$ to obtain a required BER. The bottleneck of a multihop cellular network is the location where the lowest SINR occurs. In a regular multihop cellular network, a capacity bottleneck occurs at the base station because all traffic is eventually transmitted to it. 


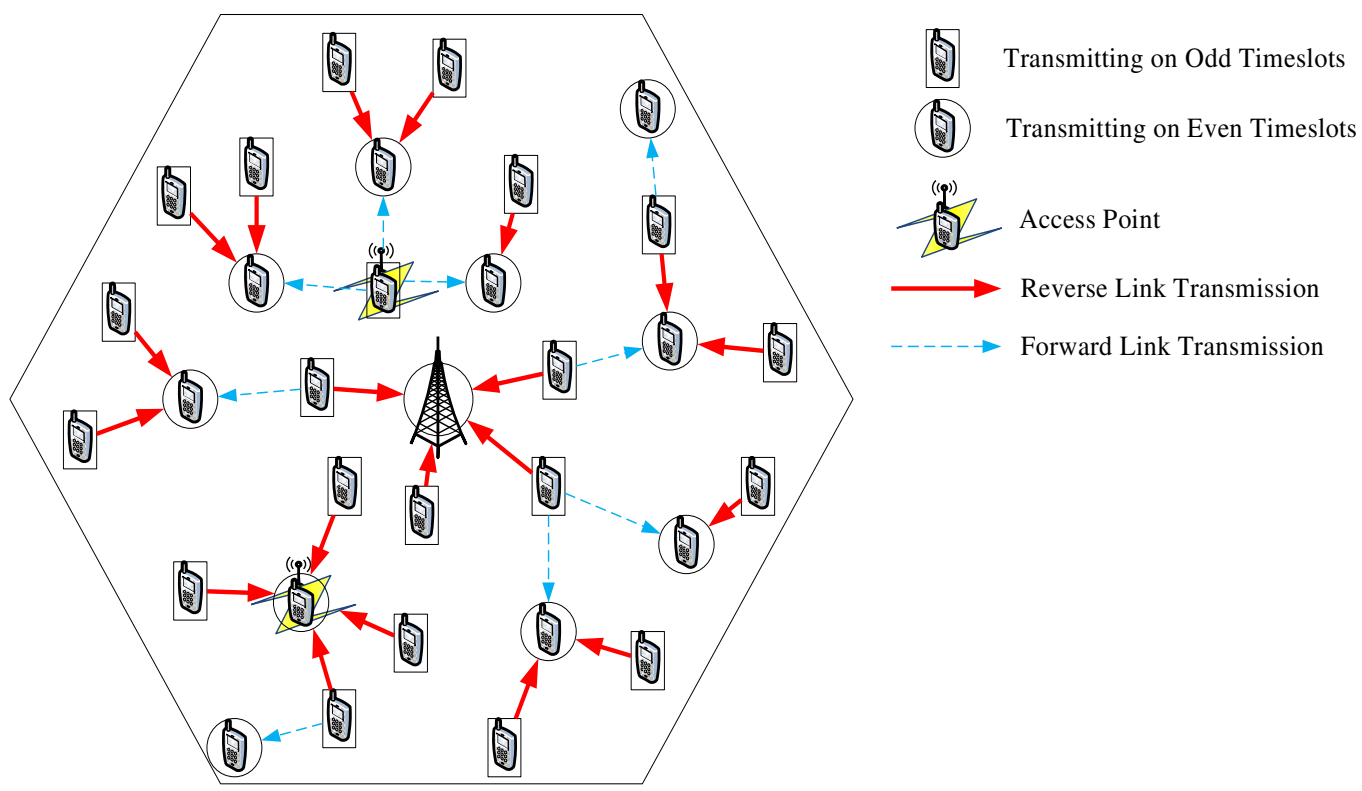

Figure 5.4: Integration of Access Points into TDD-CDMA Mechanism for Autonomous Infrastructure Multihop Cellular Networks

In an autonomous infrastructure multihop cellular network, the point of lowest SINR is still the base station because it is the location to which the greatest amount of traffic is transmitted. However access points will be able to handle some of the traffic that would normally be destined to the base station and therefore the interference at the base station will be lower.

Analysis of the SINR at the base station is now presented because it is the capacity bottleneck of the network. The probability of bit error in an autonomous infrastructure multihop cellular CDMA network is a monotonically decreasing function of the SINR:

$$
\begin{gathered}
P_{\text {error }}=f\left(\operatorname{SINR}_{\text {Autonomous }}\right) \\
P_{\text {error }}=f\left(\frac{E_{b}}{N_{0}+I}\right)
\end{gathered}
$$

Due to the careful design of the routing paths in the minimum hop routing algorithm as well as the use of TDD-CDMA, the majority of the interference at a base station 
or an access point is due to the transmissions of its children. Each of the children's transmissions has equal SINR at the receiver due to the use of power control. The only other terminals that may cause any significant amount of interference at a base station are mobile terminals that are transmitting to an access point that is within two hops of the base station. Note that terminals that transmit on even timeslots do not create interference for terminals that transmit on odd timeslots when using TDDCDMA. Transmitting terminals that are more than two hops away from the base station create minimal interference there because those terminals are not even within range of any terminal in layer 1 that transmits directly to the base station.

From Figure 5.4 we see that the sources of interference at the base station, from largest to smallest, are the interference from the base station's transmitting children, the interference from terminals transmitting directly to an access point that would be in the base station's layer 2, the interference from terminals transmitting (using two hops) to an access point that would be in the base station's layer 1 and the interference from terminals transmitting to the base station that are in its layer 3 .

Therefore, the SINR at a base station that has $M_{a}$ terminals transmitting towards the base station in its $a^{\text {th }}$ layer is:

$$
\mathrm{SINR}_{\text {Autonomous }}=\frac{E_{b}}{\frac{\Psi}{N} \sum_{1}^{M_{1}-1} E_{b}+\frac{\Psi}{N} \sum_{i=1}^{A P_{1,2}} E_{b, i}+\frac{\Psi}{N} \sum_{j=1}^{A P_{2,1}} E_{b, j}+\frac{\Psi}{N} \sum_{k=1}^{M_{3}} E_{b, k}+N_{0}}
$$

where $A P_{x, y}$ denotes the number of transmitting terminals in the $x^{\text {th }}$ layer of any access point which itself would be in the base station's $y^{\text {th }}$ layer, $E_{b}$ is the energy received at the base station from its children, $E_{b, i}$ is the energy received at the base station from the $i^{\text {th }}$ mobile terminal transmitting directly to an access point which is 2 hops away from the base station, $E_{b, j}$ is the energy received at the base station from the $j^{\text {th }}$ mobile terminal that is 2 hops away from an access point which itself is within direct transmission range of the base station and $E_{b, k}$ is the energy received at the base station from the $k^{\text {th }}$ 
transmitting mobile terminal in the base station's layer 3.

Because transmitting terminals that are more than two layers away from a receiver cause minimal interference, transmitting terminals that are in layer 3 cause a negligible amount of interference at the base station and assuming thermal noise $N_{0}$ is small compared to the co-channel interference:

$$
\operatorname{SINR}_{\text {Autonomous }} \approx \frac{E_{b}}{\frac{\Psi}{N}\left(\left(M_{1}-1\right) E_{b}+\sum_{i=1}^{A P_{1,2}} E_{b, i}+\sum_{j=1}^{A P_{2,1}} E_{b, j}\right)}
$$

We can now compare this equation with the SINR at the base station of a regular multihop cellular network found in equation 4.12 of section 4.4 where the base station has $C$ transmitting children.

$$
\operatorname{SINR}_{\mathrm{MCN}} \approx \frac{E_{b}}{\frac{\Psi}{N}(C-1) E_{b}}
$$

Because terminals only transmit to an access point or a mobile terminal if the received power of their pilot signal is greater than the base station's received pilot power as is shown in Figure 5.4, $E_{b}$ is always larger than $E_{b, i}$ and $E_{b, j}$. Furthermore, the number of children $M_{1}$ transmitting to a base station in an autonomous infrastructure multihop cellular network is much smaller than the number of children $C$ transmitting to a base station in a regular multihop cellular network. In fact, $C \geq M_{1}+A P_{1,2}+A P_{2,1}$. Because $E_{b}>E_{b, i}$ and $E_{b}>E_{b, j}$ and $C \geq M_{1}+A P_{1,2}+A P_{2,1}$, we now show that autonomous infrastructure multihop cellular networks have a lesser amount of interference at the base station and a higher SINR than regular multihop cellular networks. 


$$
\begin{aligned}
\operatorname{SINR}_{\text {Autonomous }} & \approx \frac{E_{b}}{\frac{\Psi}{N}\left(\left(M_{1}-1\right) E_{b}+\sum_{i=1}^{A P_{1,2}} E_{b, i}+\sum_{j=1}^{A P_{2,1}} E_{b, j}\right)} \\
& >\frac{E_{b}}{\frac{\Psi}{N}\left(\left(M_{1}-1\right) E_{b}+\sum_{i=1}^{A P_{1,2}} E_{b}+\sum_{j=1}^{A P_{2,1}} E_{b}\right)} \\
& =\frac{E_{b}}{\frac{\Psi}{N}\left(\left(M_{1}-1\right) E_{b}+A P_{1,2} E_{b}+A P_{2,1} E_{b}\right)} \\
& \geq \frac{E_{b}}{\frac{\Psi}{N}(C-1) E_{b}}
\end{aligned}
$$

Therefore,

$$
\mathrm{SINR}_{\text {Autonomous }}>\mathrm{SINR}_{\mathrm{MCN}}
$$

\subsection{Advantages of Autonomous Infrastructure}

In the autonomous infrastructure multihop cellular network architecture, mobile terminals that are connected to the backbone network and meet certain criteria perform the functionality of an access point and forward other terminals' traffic directly onto the backbone network. Introduction of autonomous infrastructure is advantageous in many ways. Having more access points connecting terminals to the backbone network reduces interference near the base station which is the capacity bottleneck of our system. Overall transmission power consumption is lower because traffic can be forwarded to a nearby access point instead of a far away base station. Coverage improves due to the increase in the number of links to the backbone network because mobile terminals that cannot find a path to a base station may be able to forward their traffic to an access point. Furthermore, the deployment of further base stations may not be necessary because of these access points. The scalability of our network is better because as the number of mobile terminals in the network grows there is a greater number of potential access points to connect other users to the backbone network. A greater number of access points will 
decrease the amount of traffic required to be handled by the base station resulting in an increase in network capacity.

Figure 5.5 shows how autonomous infrastructure is advantageous to a multihop cellular network. In Figure 5.5a) we see that there is one base station and a number of mobile terminals distributed in space. These terminals form a typical multihop cellular network using a tree-forming minimum hop routing algorithm [11] where mobile terminals are not used as access points. We can see that the base station has the burden of handling all the traffic from all mobile terminals. There is also a lack of coverage because three mobile terminals are not within range of any other terminal that is connected to the base station. In Figure 5.5b) we have the same distribution of terminals in space except that this is an autonomous infrastructure multihop cellular network. In this case, two terminals are within transmission range of a WLAN and become access points. The burden of handling traffic is now being shared by the access points and the base station resulting in less traffic passing through the base station. This will lead to a decrease in interference at the bottleneck of the network and an increase in overall capacity. Furthermore, coverage is improved because all users are now connected to the network. We also see that the average packet delay is lower because there are less packets being forwarded to a base station that is far away from the source terminal. Power consumption is lower because traffic does not have to travel as far to reach the backbone network.

\subsection{Modes of Operation}

There are several modes of operation that are possible when mobile terminals have the capability to perform the functionality of access points in a multihop cellular network. We outline each of these modes to show the increased flexibility of the autonomous infrastructure multihop cellular network as compared to other cellular networks.

1. Standard Mode: The standard mode is the normal mode of operation of an au- 

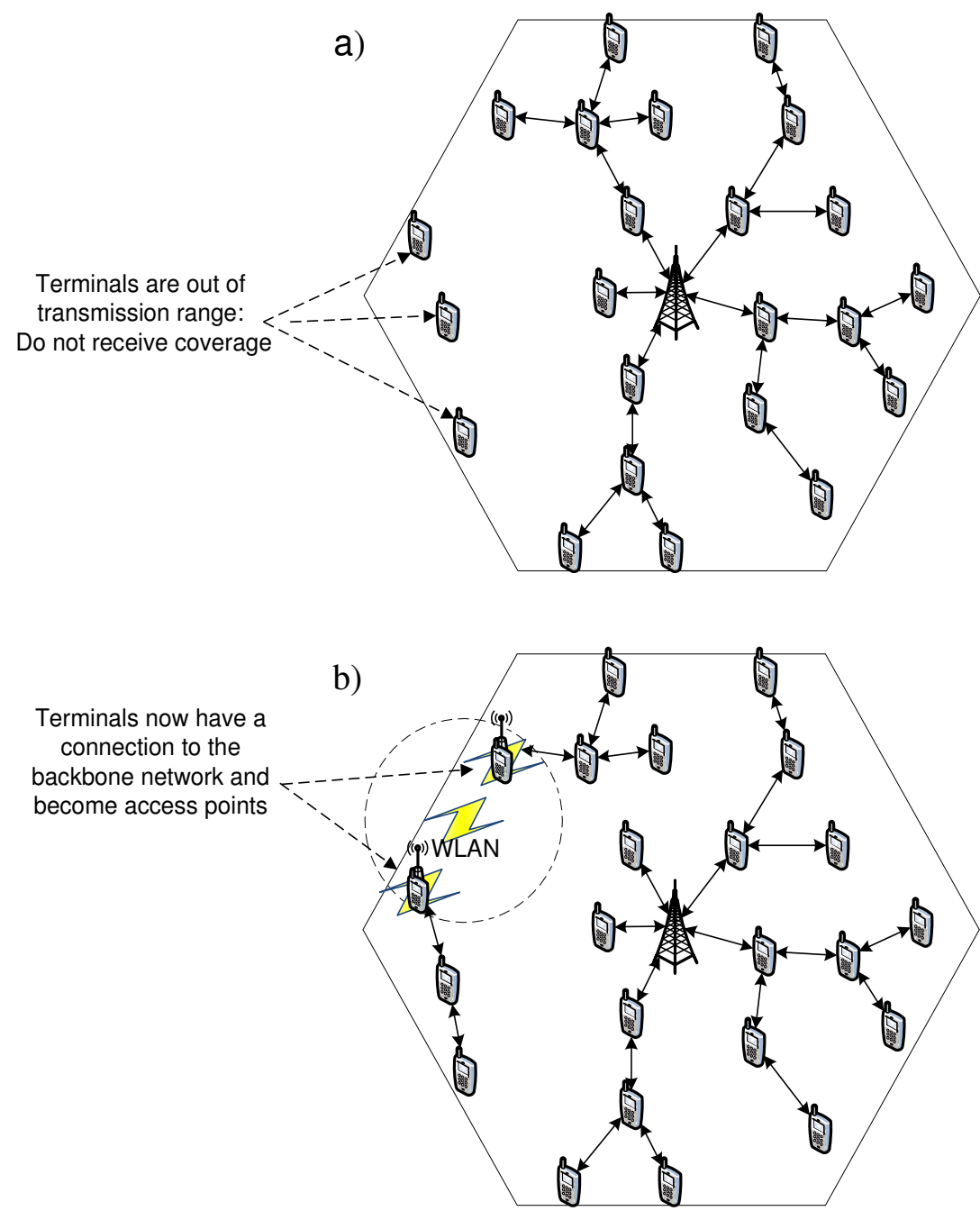

Figure 5.5: a) Regular Multihop Cellular Network b) Autonomous Infrastructure Multihop Cellular Network

tonomous infrastructure multihop cellular network that has been described in this chapter. In this mode, there are mobile terminals, base stations and access points. The base station is the primary link to the backbone network and any access point will help handle some of the traffic for the base station. Mobile terminals are used as relays to forward traffic for other terminals towards base stations and access points and vice versa.

2. Autonomous Mode: The autonomous mode can be used in the special case where there are mobile terminals and access points but a base station does not exist. This 
situation could occur when there is a base station failure or even in a rural area where a base station has not been deployed. In this mode, access points can act like a base station and use the minimum hop routing algorithm to provide service to as many terminals as they are capable of handling. In an autonomous infrastructure multihop cellular network, access points already have the capability of sending traffic directly onto the backbone network for other terminals and therefore no increase or change in functionality is required for this mode.

3. Ad Hoc Mode: The ad hoc mode can be used in the special case where there are mobile terminals but there are no base stations or access points. This situation could occur in a remote area like a cruise ship or a rural area where a base station has not been deployed. In this mode, mobile terminals can form an ad hoc network using a distributed routing algorithm like [26][49]. This is possible because mobile terminals have the capability to transmit directly to one another in a multihop cellular network. However in this mode of operation mobile terminals will require additional functionality that allows terminals to form an ad hoc network when no base stations or access points exist. The coverage area of this type of network would be limited in size for reasons stated in section 2.2.1 involving the limitations of ad hoc networks. Note that this mode of operation is possible in both regular and autonomous infrastructure multihop cellular networks.

The work in this thesis assumes the use of the Standard Mode of operation as described in the system model presented in chapter 3 and updated in section 5.3 as it is the typical mode of operation of the autonomous infrastructure multihop cellular network. The majority of this work will also apply to the Autonomous Mode as it is a special case of the Standard Mode where no base stations exist. 


\subsection{Simulation Results}

In order to quantify the advantages of autonomous infrastructure, some simulation results are presented in this section. In these simulations, the transmission range factor $K$ is 4 and the number of terminals $M$ in each cell is 200. All simulations results in this section are plotted against the access point probability $p_{a p}$. A value of $p_{a p}=0$ is equivalent to a normal multihop cellular network without autonomous infrastructure. As we increase $p_{a p}$, we increase the number of mobile terminals that can act as access points. Access points are limited to handling all transmissions of 20 mobile terminals to model their limited processing and transmit power capabilities. The model used for the simulations was presented in chapter 3 and section 5.3 and the remainder of the simulation parameters are taken from section 3.8.

Figure 5.6 shows the average SINR at the base station with respect to the access point probability. From Figure 5.6, we observe that the SINR at the base station can be increased by increasing the number of access points in the network. When ten percent of mobile terminals are access points $\left(p_{a p}=0.1\right)$, the average SINR at the base station is $7.1 \mathrm{~dB}$ higher than in a regular multihop cellular network. However, in a real network, a smaller number of mobile terminals may be connected to the backbone network. Even when $p_{a p}=0.03$, the average SINR is increased by $2.8 \mathrm{~dB}$. The reason that we achieve higher SINR is that the interference at the base station decreases as we increase $p_{a p}$. This is because a smaller amount of traffic is destined to the base station, instead being handled by access points. Having a higher SINR at the base station is important because the base station is the capacity bottleneck of the network due to the large amount of interference surrounding it.

Figure 5.7 shows the average total power consumption of all mobile terminals with respect to the access point probability. From Figure 5.7, we observe that the total power consumption decreases as we increase the number of access points in the network. When $p_{a p}=0.1$, the average power consumption is $3.3 \mathrm{~dB}$ lower than in a normal multihop 


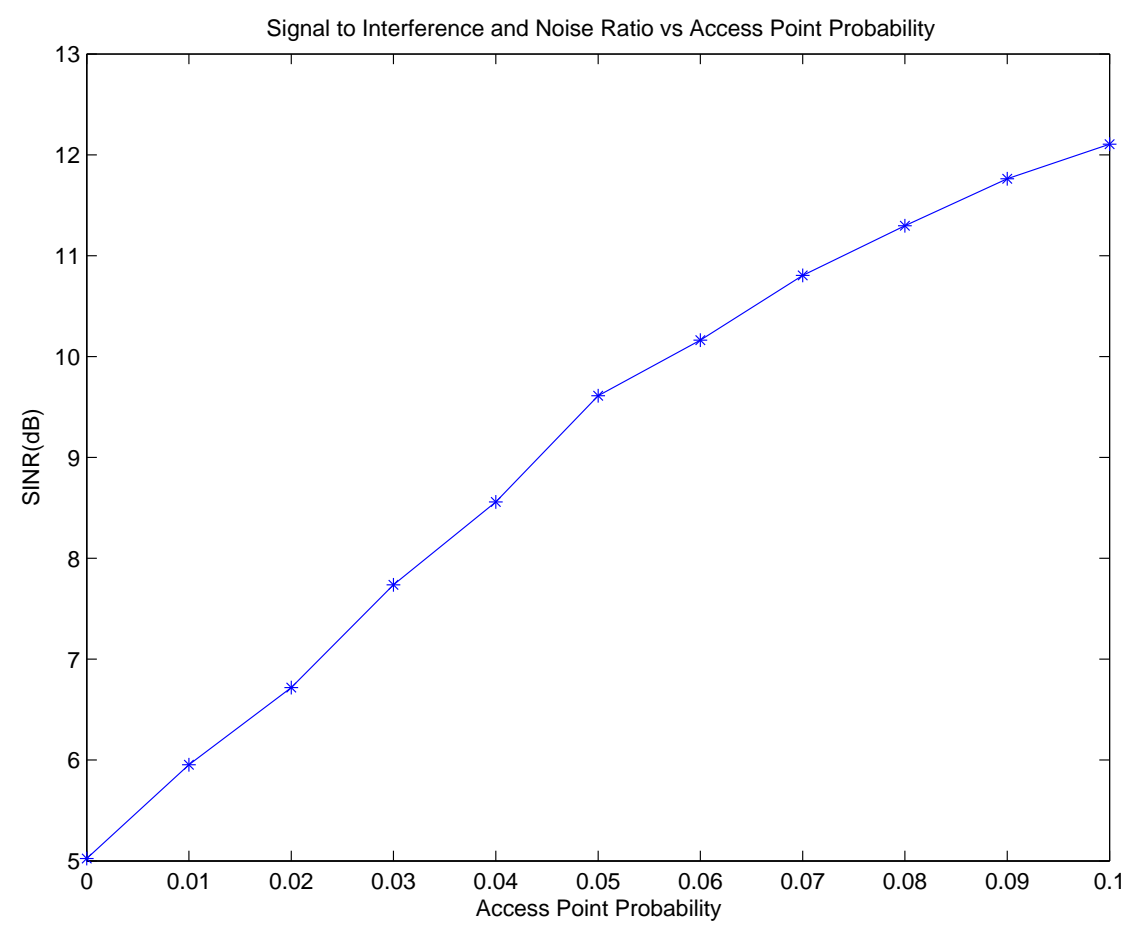

Figure 5.6: Average SINR vs Access Point Probability

cellular network. Even in a network where only three percent of mobile terminals perform the functionality of access points $\left(p_{a p}=0.03\right)$, a power savings of $1.3 \mathrm{~dB}$ can be realized. These results are as expected because by allowing mobile terminals to act as access points, a lesser number of transmissions will have to be forwarded to a far away base station. Instead transmissions will be sent to an access point that is close by. This will lead to a decrease in the number of transmissions required for each packet to reach the backbone network, and therefore a lower overall power consumption.

Figure 5.8 shows the average number of hops required for traffic emanating from a mobile terminal to reach the base station or an access point with respect to the access point probability. From Figure 5.8, we observe that the mean hop count decreases as we increase the number of access points in the network. When $p_{a p}=0.1$, the mean hop count is 1.21 as compared to 2.37 in a normal multihop cellular network. Even in a network where $p_{a p}=0.03$, the mean hop count is decreased to 1.72 . These results are as expected because increasing the number of access points will lead to less traffic being 


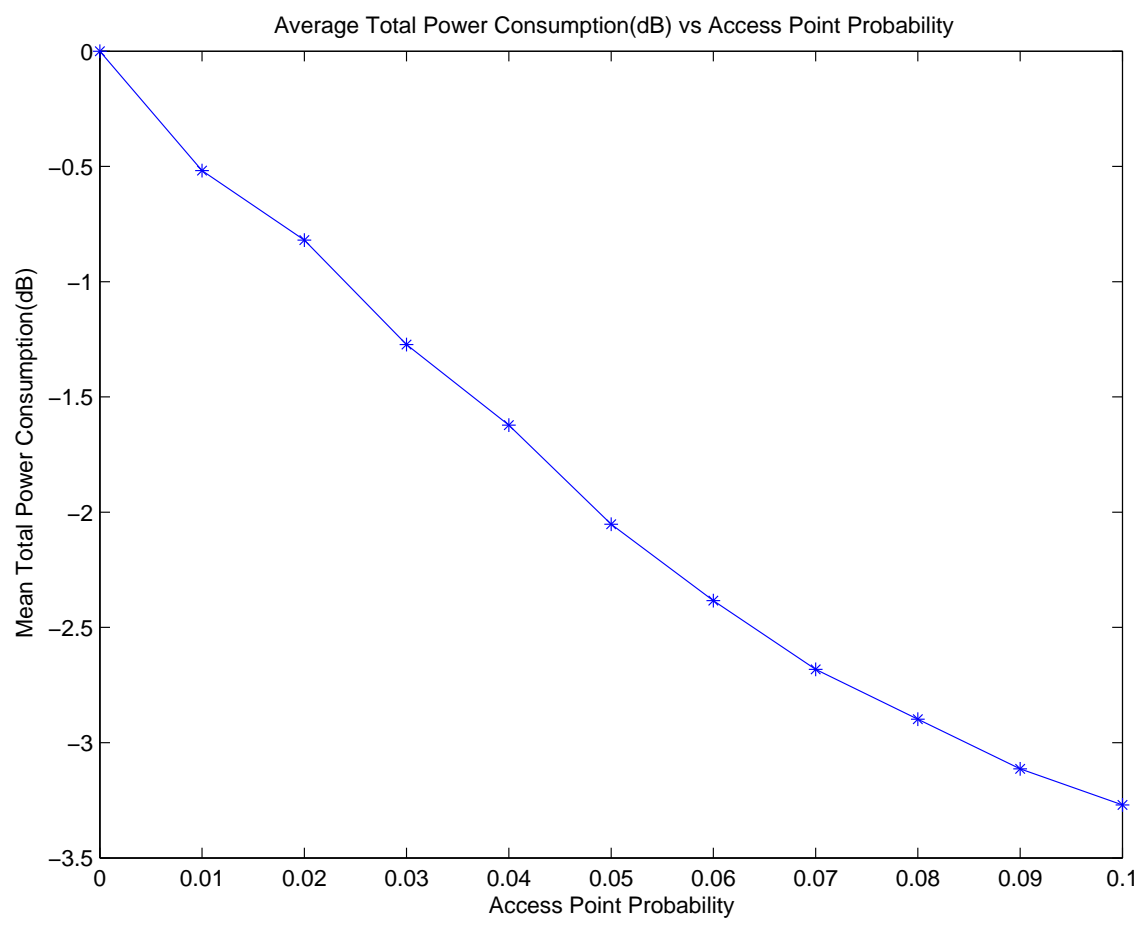

Figure 5.7: Total Power Consumption vs Access Point Probability

forwarded a large number of hops to a base station, instead being transmitted to an access point that is close by. Here we increase the range of our simulations to $p_{a p}=$ 0.2 to show that our system approaches a one hop network as we increase $p_{a p}$ to 0.01 and that further increasing the number of access points does not result in significant improvements in performance in terms of average hop count. Having a low hop count is essential because it will result in less complex routing paths making the network less vulnerable to routing path recalculations due to mobility of terminals. Having a low hop count is also important to minimize end-to-end delay.

Figure 5.9 shows the change in the coverage of mobile terminals with respect to the access point probability for a sparsely populated multihop cellular network with $M=$ 50 terminals. For this simulation we lower the number of terminals per cell from 200 to 50 to decrease the number of potential paths to the base station, making loss of coverage an issue. A terminal is considered to be uncovered when it is not possible for the terminal to find a path to the backbone network given the transmission range 


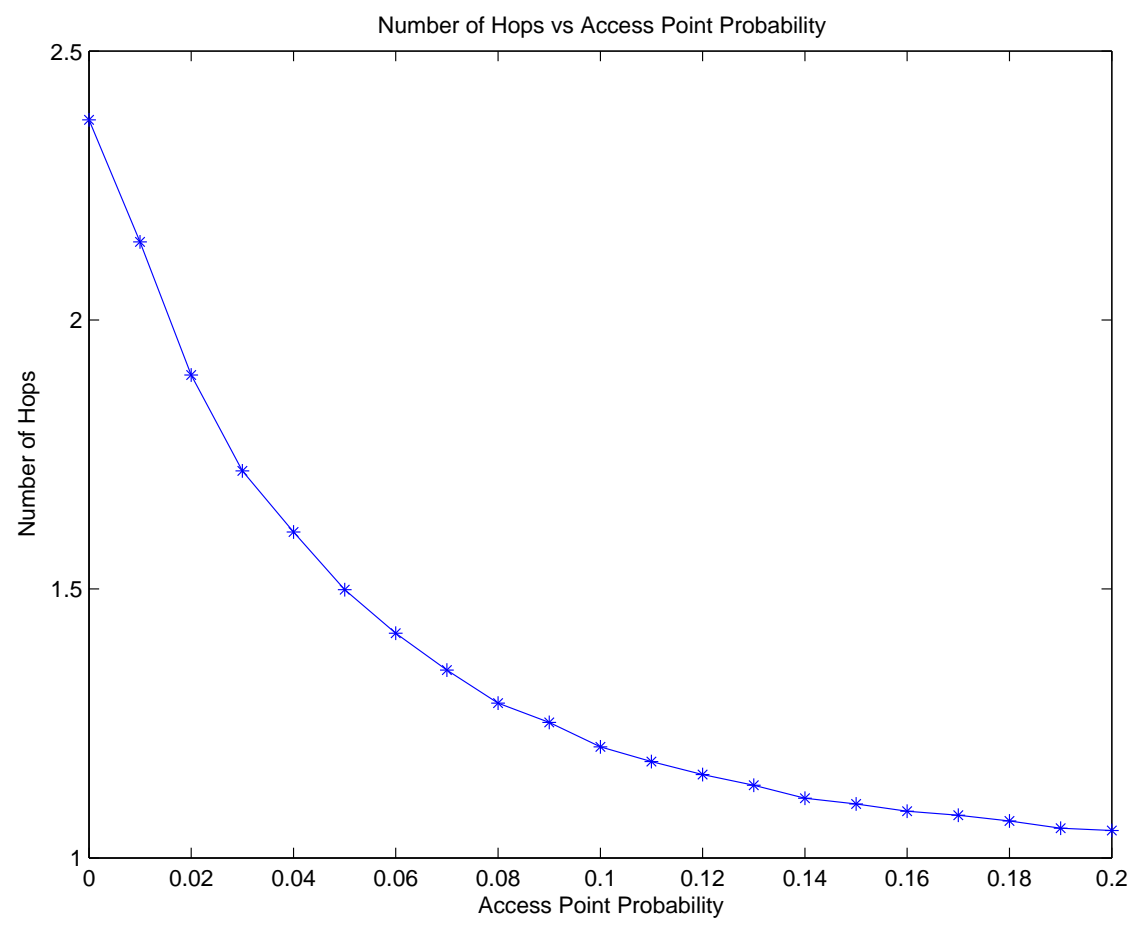

Figure 5.8: Average Hop Count vs Access Point Probability

of the mobile terminals. From Figure 5.9, we observe that the number of uncovered terminals decreases (or coverage improves) as we increase the number of access points in the network. When $p_{a p}=0.1$, the average number of uncovered terminals is $2.4 \mathrm{~dB}$ lower than in a multihop cellular network without autonomous infrastructure where $p_{a p}=0$. In a network where only three percent of mobile terminals can act as access points $\left(p_{a p}=0.03\right)$, the number of uncovered terminals is $1.0 \mathrm{~dB}$ lower. These results are as expected because by increasing $p_{a p}$ we are increasing the number of links to the backbone network. Mobile terminals that are not able to find a routing path to the base station may still be able to find a routing path to an access point. This leads to the autonomous infrastructure multihop cellular network having better coverage than a normal multihop cellular network. 


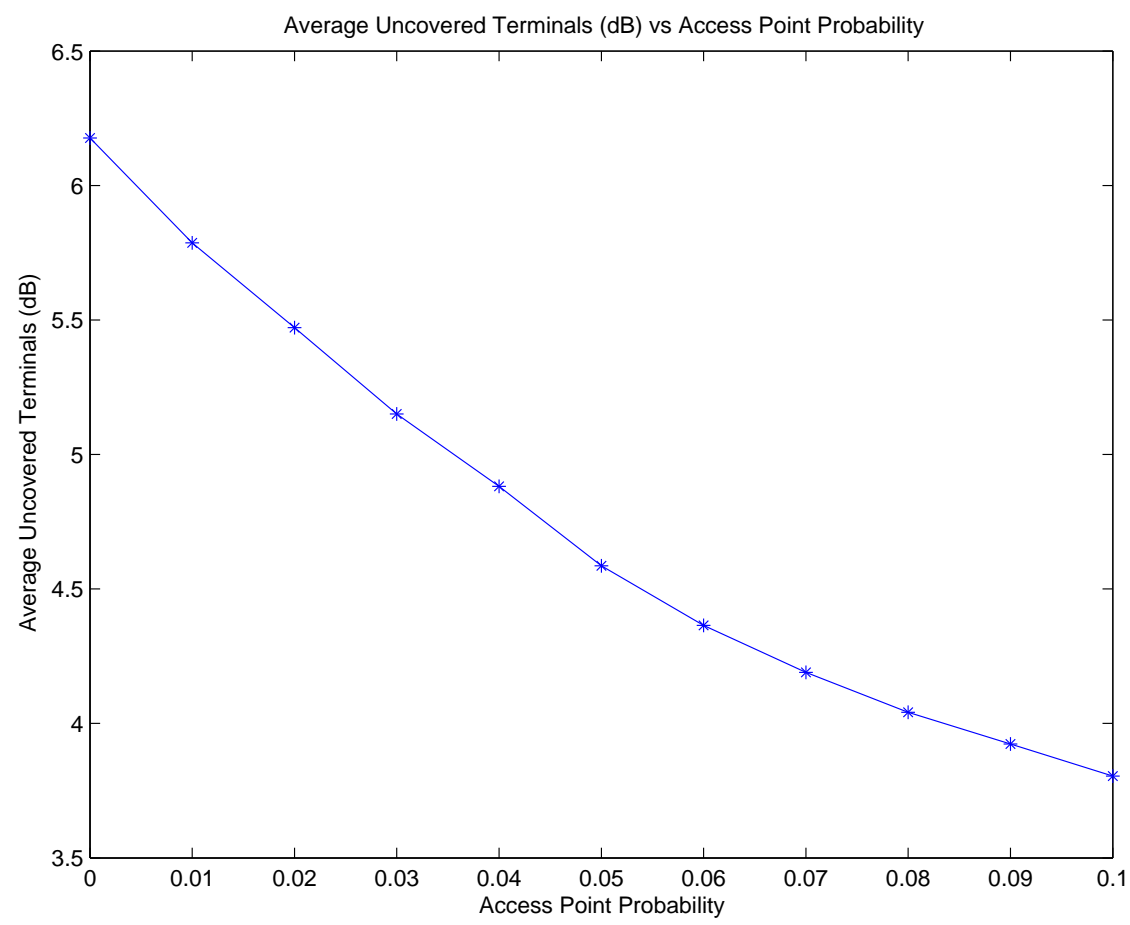

Figure 5.9: Average Uncovered Terminals vs Access Point Probability

\subsection{Chapter Summary}

In this chapter, we proposed a novel architecture for the multihop cellular network where users can connect their mobile terminals to the backbone network to act as access points and forward the traffic of other terminals directly onto the backbone network. We showed that the multihop cellular network with autonomous infrastructure is a robust flexible alternative to the traditional cellular network. Results of simulations and analysis showed that by introducing autonomous infrastructure, we obtain a higher SINR and a lower power consumption than in a normal multihop cellular network. Autonomous infrastructure multihop cellular networks were also shown to have better coverage than regular multihop cellular networks.

Our simulation results showed that by increasing the number of access points, the capacity of the network will increase. If the capacity of the network is increased, a larger number of users can be supported. With a larger number of mobile terminals, we can 
envision that more of these terminals will have links to the backbone network and will act as access points. This will lead to a further increase in capacity. Following this reasoning, we can see how this flexible network is in fact scalable and how growth of the network can occur in an organic manner. This concept is illustrated in Figure 5.10.

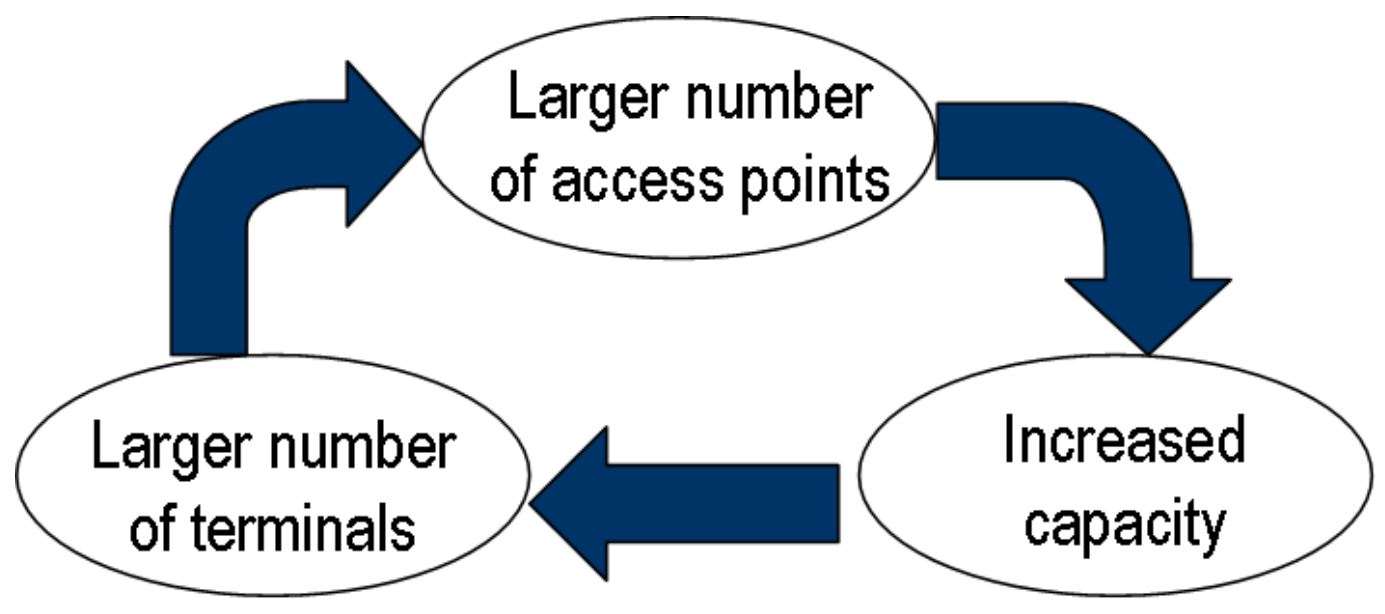

Figure 5.10: Organic Growth of Network

This architecture is advantageous to the network operator because it will not need to invest in the deployment of costly infrastructure. Moreover, the network operator may be provided with more traffic, leading to more revenue due to the network's higher capacity. The network operator will only be responsible for managing the network domain controller which is in charge of security and billing issues. This proposed networking concept is revolutionary in the sense that it puts the control of infrastructure deployment partly in the hands of the user. This could have the effect of stimulating the deployment of future wireless services. 


\section{Chapter 6}

\section{Pilot Power Protocol for Optimal Transmission Range}

Access points require parameters like their transmission ranges to be adjusted autonomously to optimal levels. We begin this chapter by presenting several simulation results that motivate the development of a pilot power protocol. We then propose a pilot power protocol (PPP) that autonomously adjusts transmission ranges to optimal levels for a given coverage requirement. The protocol is said to be optimal if it determines the transmission range that maximizes SINR and minimizes power consumption given the constraint of a coverage requirement. A quantitative analysis of the pilot protocol in an autonomous infrastructure multihop cellular network is then presented. We then present simulation results that show that the pilot power protocol obtains better performance in terms of SINR, power consumption and coverage than when constant maximum transmission ranges are used. We also show that the bottleneck that exists at the base station in a regular multihop cellular network does not occur in the autonomous infrastructure multihop cellular network with the pilot power protocol because mobile terminals can be added to the network without decreasing the SINR at the base station. Our results also show that by adjusting a parameter within the pilot power protocol, a required percentage of 
covered terminals can be specified and achieved by the network without prior knowledge of the location or density of mobile terminals. Furthermore, we show through analysis and simulation that the pilot power protocol determines the pilot power level which is optimal in terms of SINR and power consumption for a given coverage requirement.

\subsection{Motivation}

A necessary feature of the proposed architecture is the automatic configuration of the transmission parameters of access points. In a traditional cellular network, radio frequency $(\mathrm{RF})$ specialists monitor base station transmissions due to regulatory requirements and coverage and interference considerations. Because the exact locations of access points are unknown and can change frequently, it is not possible for RF specialists to adjust the transmission parameters of access points. Access points therefore have to determine their optimal transmission parameters autonomously.

An access point has a limited capability depending on the mobile terminal's battery power and the bandwidth of its connection to the backbone network and is therefore only able to handle traffic for a limited number of terminals that are a certain number of hops away. An access point also has hardware limitations due to its power amplifier and its processing capability that restrict its maximum transmission power level $P_{M A X}$. The access point will have to autonomously choose an optimal maximum transmission range that is high enough to ensure coverage of a sufficient number of terminals but not excessively high that it is causing too much interference. Clearly, the chosen maximum transmission range must involve the use of a transmission power level that is below the maximum transmission power level dictated by the hardware limitations. In our architecture access points and the terminals they are serving have the same transmission range to ensure that bidirectional communication is possible. The transmission range is $\frac{R}{K}$ and therefore transmission ranges can be modified by adjusting the transmission range 
factor $K$. Having an optimally chosen transmission range is essential because it has a crucial effect on the number of hops required for traffic emanating from a terminal to reach the backbone network. This is because all terminals that receive and can decode an access point's pilot signal transmit directly to an access point and are required to act as relays for other terminals that do not receive an access point's pilot signal. The transmission range of access points and terminals is also instrumental in determining the percentage of terminals that receive coverage in a cell. Therefore determining an optimal maximum transmission range is essential as it plays a crucial role in the transmission patterns, interference levels, power consumption and coverage levels in an autonomous infrastructure multihop cellular network.

An objective of this research is to develop a protocol that autonomously determines the optimal transmission range of access points and terminals for a given coverage requirement. In our architecture, an access point is restricted from transmitting data with a transmission power that is higher than its pilot power level. This implies that the transmission range of the access point is defined by its pilot power level. This is because only terminals that receive and can decode an access point's pilot signal transmit directly to that access point and are said to be within transmission range. This implies that we can meet our objective of optimizing the transmission range of mobile terminals by adjusting the pilot power levels of access points to optimal levels.

In section 6.2, we develop and present a pilot power protocol for the multihop cellular network which is used to determine optimal pilot power levels for both access points and base stations based on the density and spatial configuration of mobile terminals within a cell [14]. Although this algorithm was developed for use by access points in the autonomous infrastructure multihop cellular network, it can equally be used for base stations in both autonomous infrastructure and regular multihop cellular networks. The pilot power level of an access point is directly related to its transmission range. This protocol optimizes the pilot power of access points and in doing so determines the 
optimal transmission range for both mobile terminals and access points [13]. Access points and base stations are expected to function optimally in cells with either a high or low density of mobile terminals. They do not have any prior knowledge of the density or location of mobile terminals surrounding them and are required to autonomously set their pilot power levels to obtain a specified required coverage level while also obtaining as low power consumption and as high SINR as possible.

To motivate this protocol we present results of several simulations performed on an autonomous infrastructure multihop cellular network with $p_{a p}=0.05$. The model used for the simulations in this chapter was presented in chapter 3 and section 5.3 and the remainder of the simulation parameters are taken from section 3.8. Figure 6.1 shows the percentage of covered terminals with respect to the transmission range factor $K$. A terminal is considered to be covered when it is possible for the terminal to find a path to the backbone network given the transmission range of the mobile terminals. As we increase $K$, we decrease the pilot power and the maximum transmission range of terminals and increase the number of hops required for a packet from a given mobile terminal to reach an access point. These results indicate that the coverage within the network decreases as we increase $K$. Figure 6.1 also shows that cells more densely populated by mobile terminals can handle lower transmission ranges than sparsely populated cells while still maintaining high coverage. This is because there are a larger number of potential paths towards a base station or access point.

On the other hand, simulation results show that the SINR increases and the power consumption per terminal decreases as we increase $K$. This can be seen in Figure 6.2 which shows the SINR with respect to $K$ and Figure 6.3 which shows the total average power consumption per covered terminal with respect to $K$. The SINR at the base station increases as we increase $K$ because using lower transmission ranges decreases the amount of in-cell as well as intercell interference at the base station. Power consumption decreases as we increase $K$ because the transmit power of each terminal is lower. Although more 


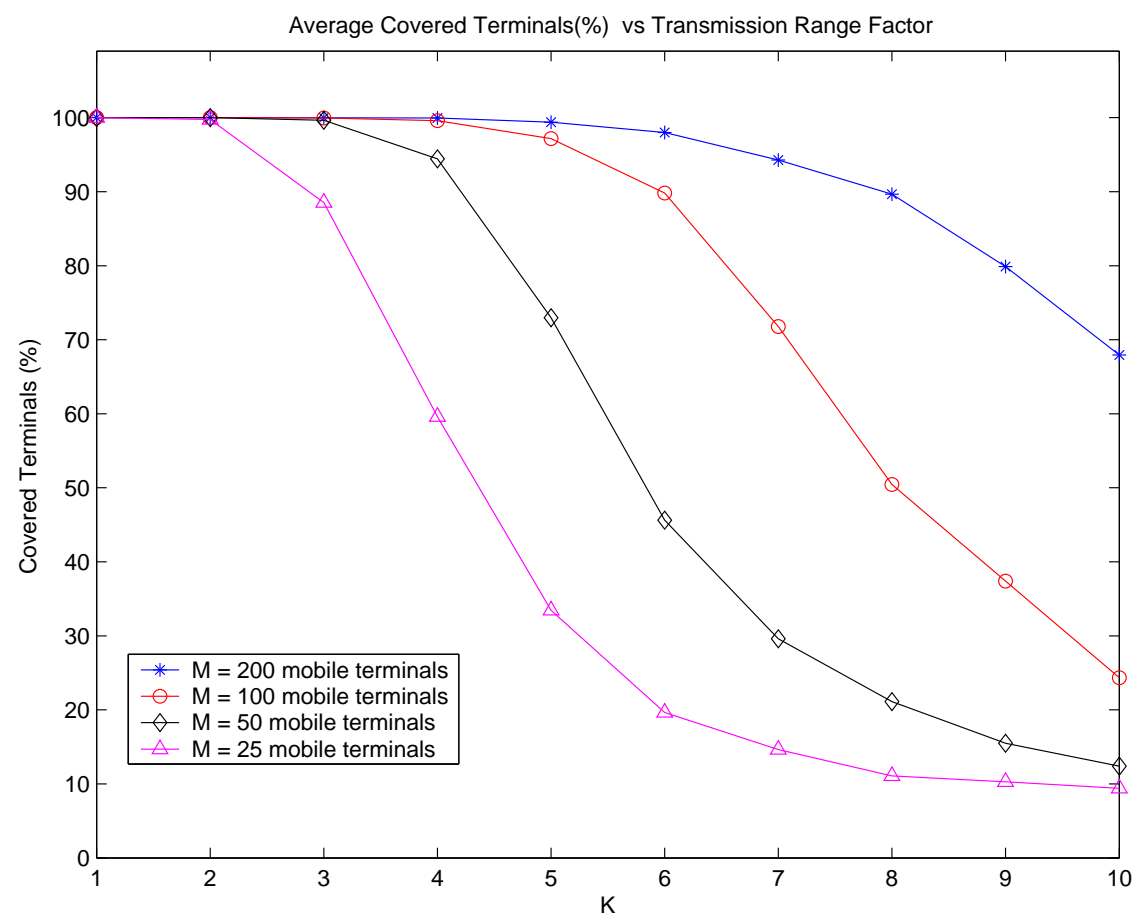

Figure 6.1: Percentage Covered Terminals vs Transmission Range Factor

transmissions are required for traffic to reach its destination, the reduction of path loss allows us to reduce the transmit power sufficiently so that the average power consumption per terminal is lower when using a larger $K$.

These results imply that there is a tradeoff of coverage vs SINR and power consumption that needs to be made. The optimization problem of pilot power levels in traditional cellular networks is currently a hot research topic [64][82]. However, there has not been any research performed on this issue for multihop cellular networks. For this reason, the majority of the works on multihop cellular networks assume the use of a constant pilot power level leading to terminals and base stations having constant maximum transmission ranges $[68][12][73]$. This is in spite of the fact that simulation results show that constant pilot powers will lead to bad performance when the density of terminals in a cell can vary. For this reason, the pilot power protocol will be used by access points and base stations to determine their optimal pilot power level, thus their optimal transmission range. The algorithm autonomously increases transmission ranges in sparsely populated cells to 


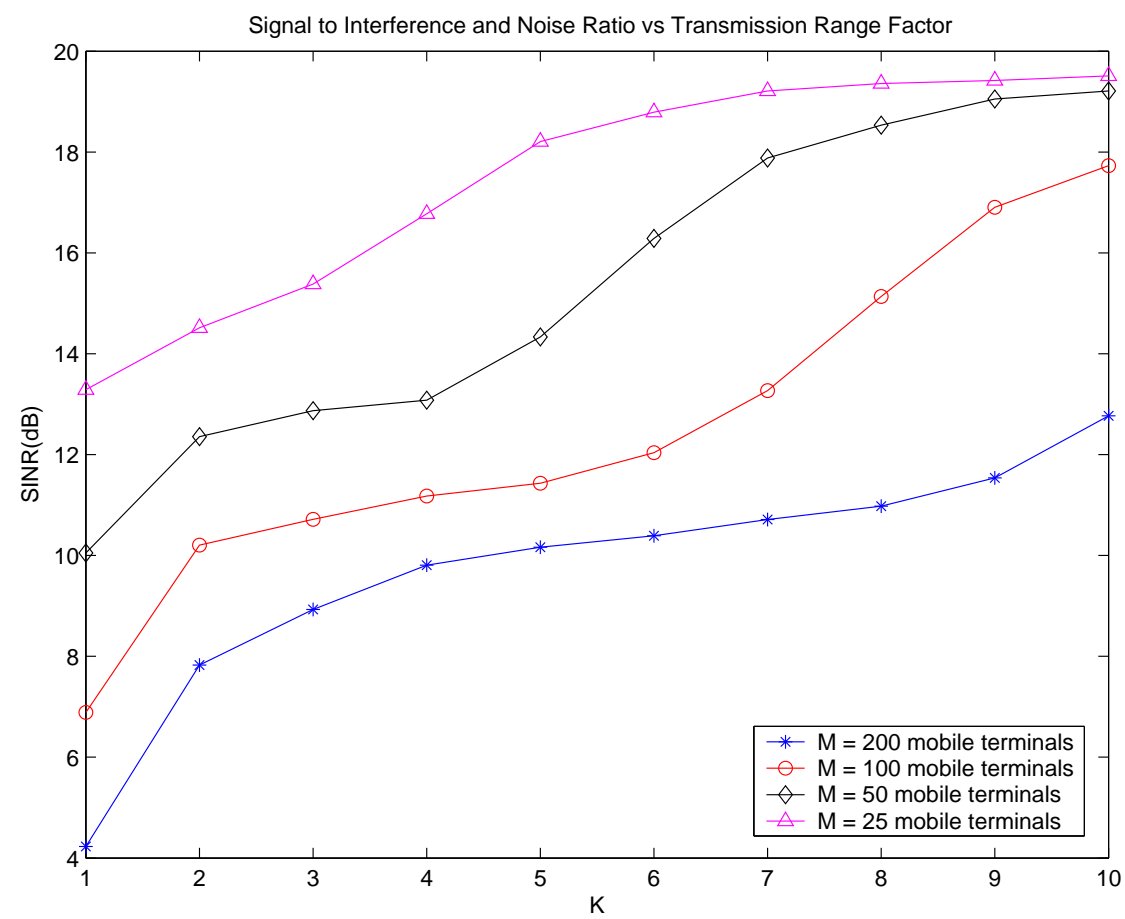

Figure 6.2: Average SINR vs Transmission Range Factor

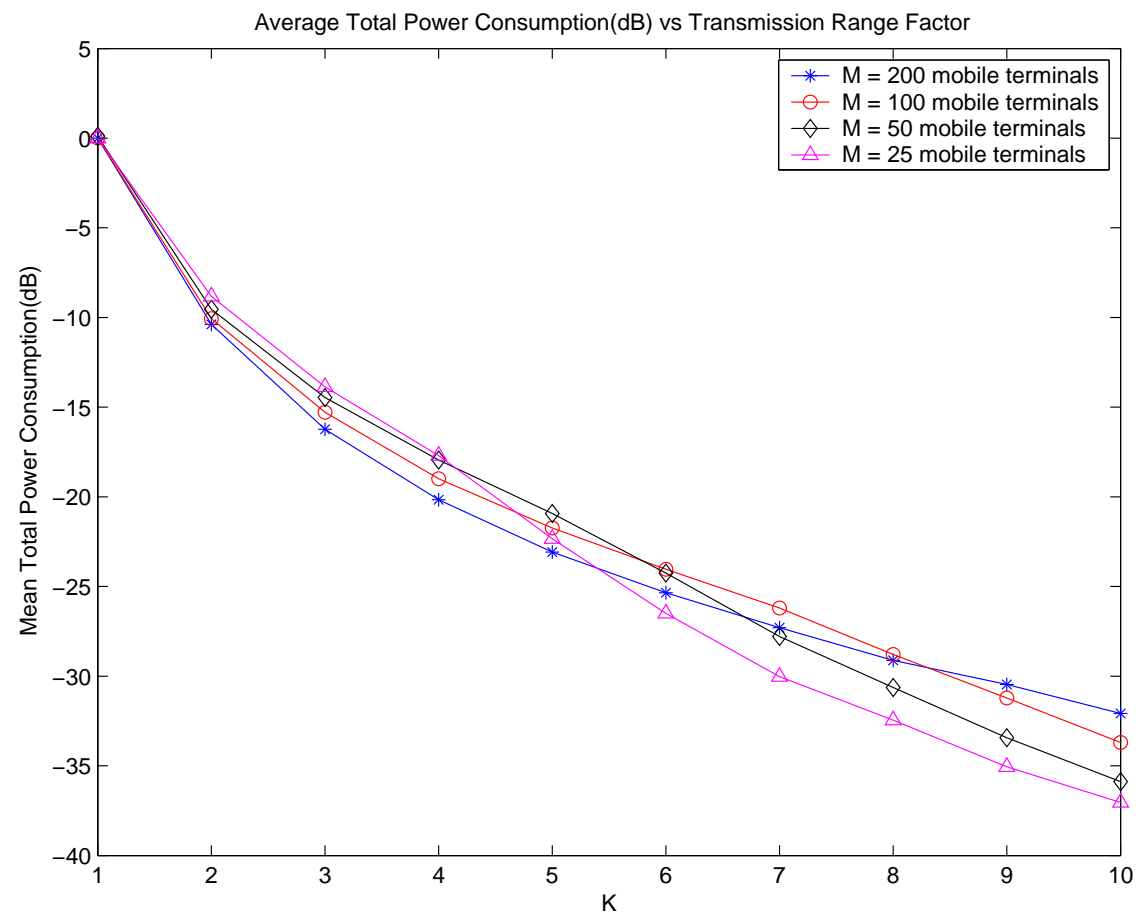

Figure 6.3: Power Consumption per Mobile Terminal vs Transmission Range Factor 
Chapter 6. Pilot Power Protocol for Optimal Transmission Range 96

achieve the required coverage level and autonomously decreases transmission ranges in densely populated cells to improve SINR and power consumption while maintaining the required coverage level.

\subsection{Pilot Power Protocol for Autonomous Infrastruc- ture Multihop Cellular Networks}

In order to ensure proper coverage in a cell, an access point must transmit its pilot signal at a power that is sufficiently high that a mobile terminal is found in the outer $50 \%$ of its range. This is because two consecutive hops where no terminal is found in the outer $50 \%$ of the transmission range results in a loss of further connectivity through that path. This is illustrated in Figure 6.4 where the access point has transmission range $r$. The access point finds mobile terminal A that will act as a relay but it is not in the outer $50 \%$ of its transmission range. The shaded area between $\frac{r}{2}$ and $r$ shows the outer $50 \%$ of the access point's transmission range. However there are not any mobile terminals that can act as relays in that area. Because the inner $50 \%$ of mobile terminal A's transmission range is bound by the access point's transmission range, it is impossible for it to find any children in that area. If mobile terminal $\mathrm{A}$ is also not able to find a terminal in the outer $50 \%$ of its transmission range as is the case in Figure 6.4, connectivity is lost in that direction. Because the pilot power and transmission ranges of terminals are too low for the density of terminals in this cell, mobile terminal B is not covered by the network. Note that in a multihop network with multiple terminals each having options to create paths as well as lognormal fading which creates path diversity, having two terminals in a row that do not push out over $50 \%$ does not necessarily imply that connectivity is lost. However, to guarantee connectivity with a certain confidence, we want to find a terminal in the outer $50 \%$ of the transmission range with a certain probability.

To have an efficient network that is adequately covered, we not only want to find one 


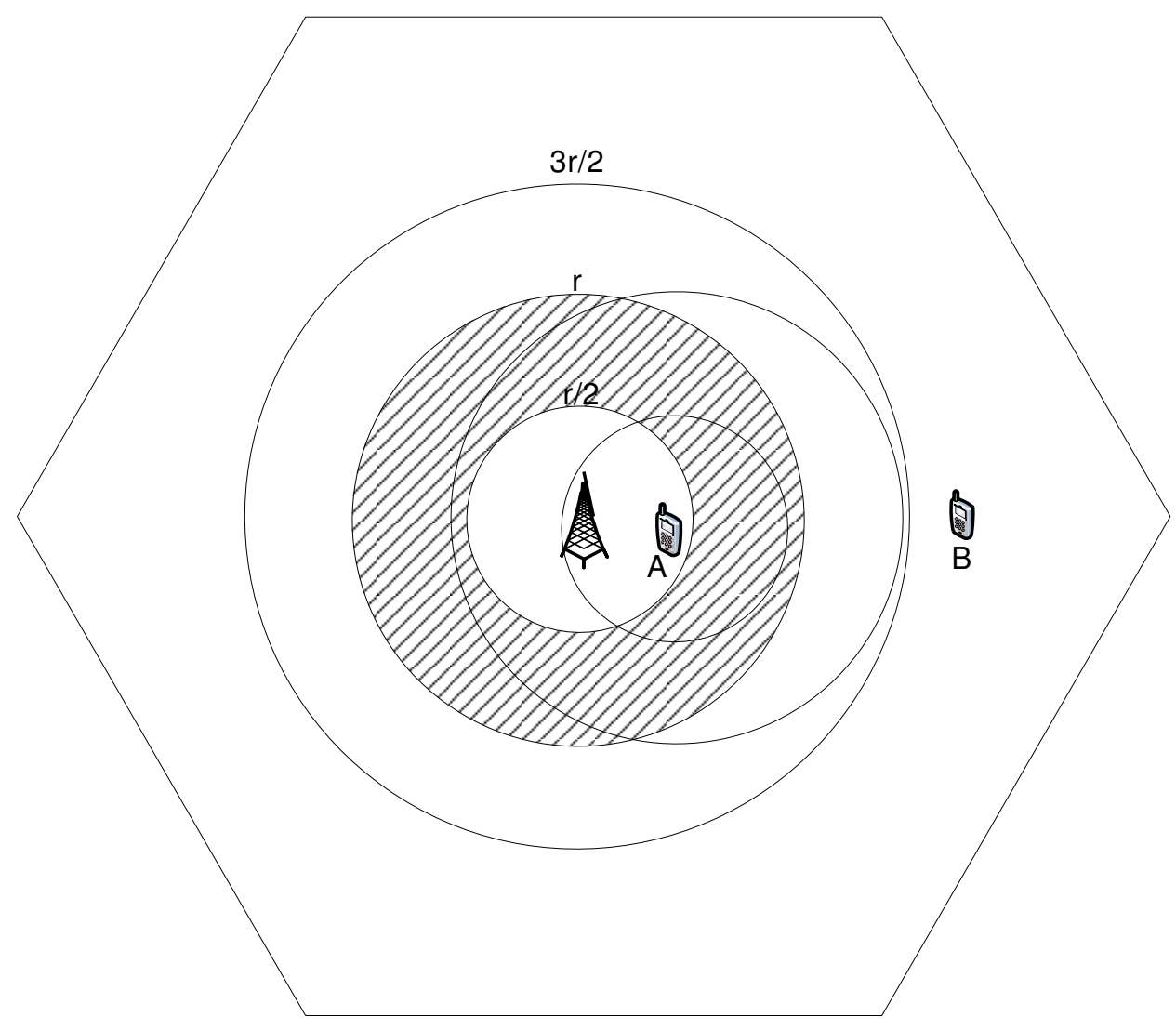

Figure 6.4: Example Showing Loss of Connectivity

mobile terminal in the outer $50 \%$ of the transmission range but we want to find mobile terminals in the outer $50 \%$ in several different directions. To achieve this we break up the area covered by the transmission range into 3 sectors as is generally the standard in CDMA systems where base stations are modeled as being placed in an hexagonal pattern. The division of the transmission range into 3 sectors is shown in Figure 6.5.

The area $S$ is found to be:

$$
\text { Area } S=\frac{1}{3}\left(\pi r^{2}-\pi\left(\frac{r}{2}\right)^{2}\right)=\frac{\pi r^{2}}{4}
$$

or $\frac{1}{4}$ of the total area covered by the access point. In this analysis we would like the access point to find at least one terminal in the outer $50 \%$ of its transmission range in each sector with probability $\gamma$. To achieve this we need to choose $r$ such that the probability that a terminal is in the shaded area $S$ is $\gamma$. However, the access point will 


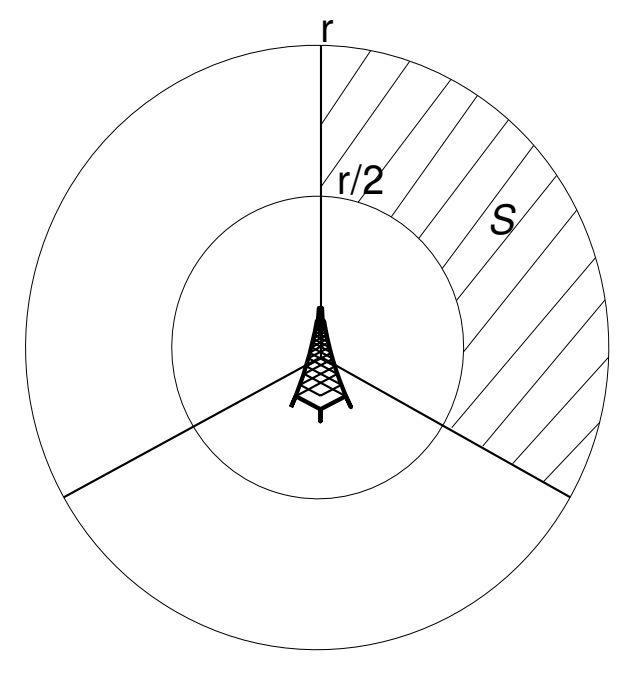

Figure 6.5: Dividing Transmission Range into 3 Sectors

not know the exact physical locations of mobile terminals. By setting $r$ such that there is a sufficient number of terminals $t$ within the range of its pilot signal, it can be certain with probability $\gamma$ that there is a terminal within area $S$. For our pilot power protocol, we first need to find the required number of terminals $t$ within transmission range of the access point such that the probability that a terminal is within area $S$ is greater or equal to $\gamma$.

$$
\operatorname{Pr}(\text { at least one terminal in } S \mid \mathrm{t} \text { terminals in range) } \geq \gamma
$$

Because terminals are randomly distributed with a uniformly distributed probability distribution function:

$$
\operatorname{Pr}(\text { at least one terminal in } S \mid \mathrm{t} \text { terminals in range })=1-\left(1-\frac{1}{4}\right)^{t}
$$

Substituting equation 6.3 into equation 6.2 gives:

$$
1-\left(\frac{3}{4}\right)^{t} \geq \gamma
$$

Simplifying equation 6.4 and solving for $t$ gives:

$$
\log (1-\gamma) \geq t \cdot \log \left(\frac{3}{4}\right)
$$




$$
t \geq \frac{\log (1-\gamma)}{\log \left(\frac{3}{4}\right)}
$$

Using this equation the required number of terminals $t$ within transmission range of an access point can be calculated for a specified $\gamma$. The access point will then set its transmission range $r$ such that there are t terminals within its range to guarantee that the probability that a terminal is in area $S$ is greater or equal to $\gamma$.

The pilot power protocol will function as follows. The network operator will have specified a percentage of terminals denoted $V$ that are required to be covered by the network. Using equation 6.18 found in section 6.4 which relates $\gamma$ and $V$, the value of $\gamma$ is calculated from $V$. The access point will then calculate the minimum number of terminals $t$ required to be within its transmission range using equation 6.6 and $\gamma$. Initially the pilot power level, denoted $P_{\text {pilot }}$, will be set to a very low initial power $P_{0}$ and it will begin transmitting. We denote $T\left(P_{\text {pilot }}\right)$ as the number of terminals within the transmission range of the access point when it is transmitting at pilot power $P_{\text {pilot }}$. The access point will then check to see if the number of terminals within its transmission range, $T\left(P_{\text {pilot }}\right)$, is greater or equal to $t$. If it is within transmission range of at least $t$ mobile terminals, the pilot power protocol is complete and the maximum transmission power of the access point and the terminals it is serving will be $P_{\text {pilot }}$. If it is not, the access point will continue incrementing its transmission power $P_{\text {pilot }}$ by a power increment $P_{\text {inc }}$ until it finds that it is within transmission range of the required number of terminals $t$. Note that the pilot power can only be increased to a maximum transmission power, $P_{M A X}$ allowed for the access point due to hardware restrictions and interference considerations. At that time the pilot power protocol is complete and the maximum transmission power of the access point and the mobile terminals it is serving will be $P_{\text {pilot }}$. Algorithm 1 shows a description of the pilot power protocol. 


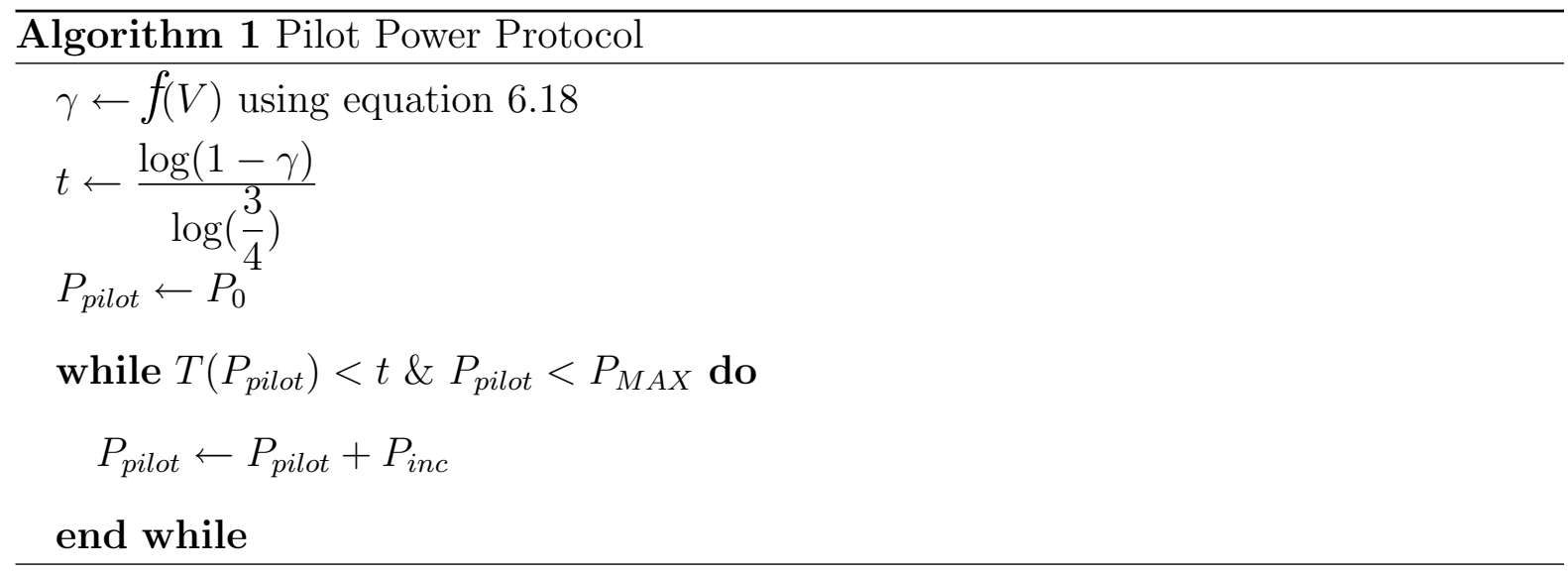

\subsection{Analytical Results}

In this section we find an analytical expression for the transmission range factor $K$ such that the number of terminals within transmission range of the base station is $t$ given that there are $M$ mobile terminals in a cell of radius $R$. For a simplistic model where lognormal shadowing is not taken into account this can be found by simply taking the ratio of the area of the transmission range to that of the cell:

$$
\begin{gathered}
t=M \cdot\left(\frac{\pi\left(\frac{R}{K}\right)^{2}}{\pi R^{2}}\right)=\frac{M}{K^{2}} \\
K=\sqrt{\frac{M}{t}}
\end{gathered}
$$

However our model incorporates lognormal shadowing:

$$
10 \log _{10}(P(d))=10 \log _{10}\left(P_{\text {area }}(d)\right)+\xi_{(0, \sigma)}
$$

where $P(d)$ is the actual received power for a terminal at distance $d$ from the access point, $\xi_{(0, \sigma)}$ is a Gaussian random variable with zero mean and standard deviation $\sigma$ in $\mathrm{dB}$, and $P_{\text {area }}(d)$, the area mean power for a terminal at distance $d$, is:

$$
P_{\text {area }}(d)=P_{t} \cdot d^{-l}
$$


where $P_{t}$ is the transmitted power and $l$ is the path loss exponent. Let us assume that for correct reception $P(d)$ must be greater than some threshold $\tau$ required to obtain SINR $>\alpha$. Define $r$, the transmission range as the distance where $P_{\text {area }}(r)=\tau[22]$.

$$
P_{\text {area }}(r)=P_{t} \cdot r^{-l}=\tau
$$

If we divide $P(d)$ by $\tau$ in equation 6.9 the result is

$$
\begin{aligned}
10 \log _{10}\left(\frac{P(d)}{\tau}\right) & =10 \log _{10}\left(\frac{P_{\text {area }}(d)}{\tau}\right)+\xi_{(0, \sigma)} \\
& =10 \log _{10}\left(\frac{P_{t} d^{-l}}{P_{t} r^{-l}}\right)+\xi_{(0, \sigma)} \\
& =10 \log _{10}\left(\frac{d}{r}\right)^{-l}+\xi_{(0, \sigma)}
\end{aligned}
$$

We can see that $10 \log _{10}\left(\frac{P(d)}{\tau}\right)$ has a Gaussian distribution with mean $10 \log _{10}\left(\frac{d}{r}\right)^{-l}$ and standard deviation $\sigma$. To correctly receive, $\frac{P(d)}{\tau}$ must be greater than 1 or $\log _{10}\left(\frac{P(d)}{\tau}\right)>$ 0 . We define $\bar{d}=\frac{d}{r}$ as the normalized distance. We also normalize other distance based variables in the cell by dividing them by the transmission range $r$ as shown in Figure 6.6.

Normalizing variables in a) by dividing them all by $r$ gives normalized variables shown in $b$ )

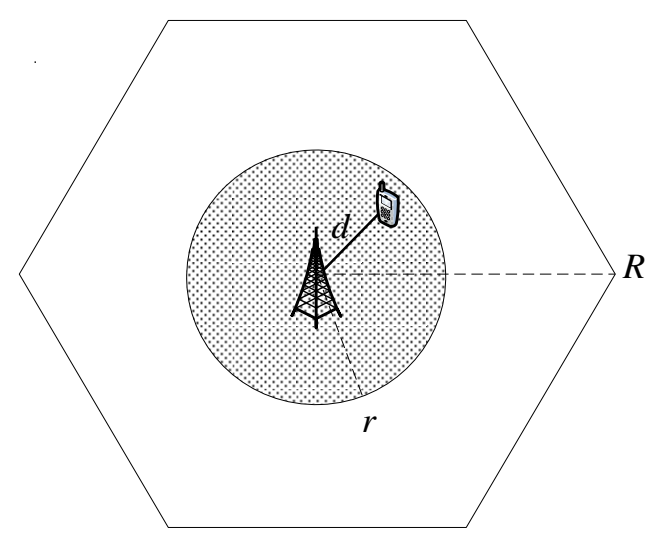

a)

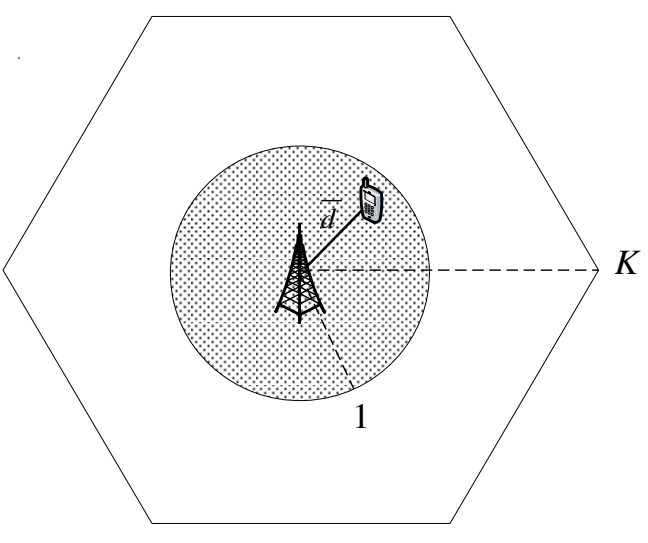

b)

Figure 6.6: Distance Metrics in a Multihop Cellular Network: a) Ordinary Metrics b) Normalized Metrics 
The probability that a mobile terminal is within transmission range of an access point given that they are separated by a normalized distance $\bar{d}$ is [22]:

$$
\begin{aligned}
\operatorname{Pr}(\bar{d}) & =\operatorname{Pr}\left[10 \log _{10}\left(\frac{P(d)}{\tau}\right)>0\right] \\
& =\frac{1}{\sqrt{2 \pi} \sigma} \int_{0}^{\infty} \exp \left(\frac{-\left(t-10 \log _{10}\left(\bar{d}^{-l}\right)\right)^{2}}{2 \sigma^{2}}\right) \mathrm{d} t \\
& =\frac{1}{2}\left(1-\operatorname{erf}\left(\frac{10}{\sqrt{2} \ln (10)} \cdot \frac{\ln (\bar{d})}{\frac{\sigma}{l}}\right)\right)
\end{aligned}
$$

Now we must find the probability that a given terminal is at distance $\bar{d}$ from the access point. Because we are using normalized distances we must also normalize the radius of the cell $R$ as shown in Figure 6.6. The normalized radius is found by dividing $R$ by the transmission range $r$ which is equal to $K$, the transmission range factor. Because mobile terminals are randomly distributed the probability distribution function of $\bar{d}$ can be found by taking the area of a small annulus surrounding $\bar{d}$, shown in Figure 6.7, and dividing it by the total normalized area of the cell.

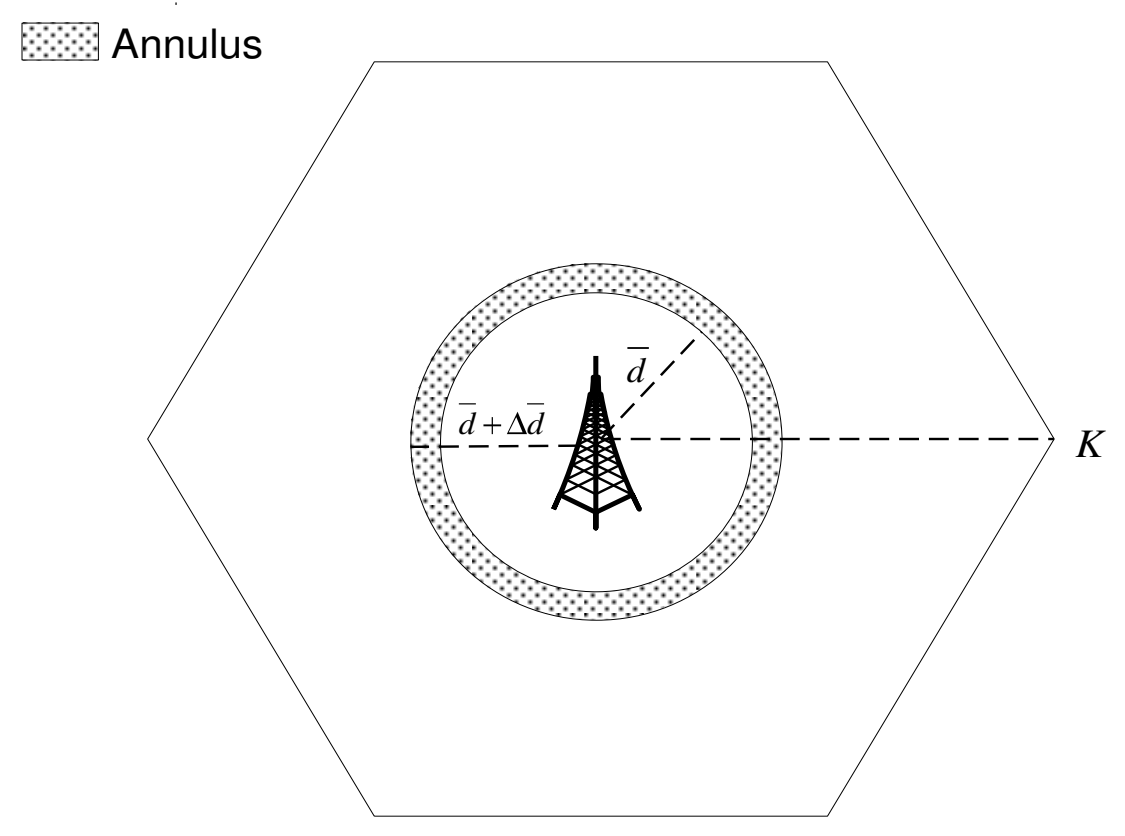

Figure 6.7: Annulus Surrounding Normalized Distance $\bar{d}$ 


$$
\begin{aligned}
& \operatorname{Pr}(\text { terminal } \in(\bar{d}, \bar{d}+\Delta \bar{d}))=\frac{\text { Area of Annulus }}{\text { Total Normalized Area of Cell }} \\
&=\frac{\pi(\bar{d}+\Delta \bar{d})^{2}-\pi \bar{d}^{2}}{\pi K^{2}} \\
&=\frac{2 \bar{d} \Delta \bar{d}}{K^{2}} \\
& f(\bar{d})= \begin{cases}\frac{2 \bar{d}}{K^{2}} & \text { if } 0 \leq \bar{d} \leq K \\
0 & \text { otherwise }\end{cases}
\end{aligned}
$$

The probability that a randomly distributed terminal is within range of the base station is equal to the integral of the probability that a terminal at distance $\bar{d}$ is within transmission range times the probability that a terminal is at distance $\bar{d}$.

$$
=\int_{0}^{K} \frac{\bar{d}}{K^{2}}\left(1-\operatorname{erf}\left(\frac{10}{\sqrt{2} \ln (10)} \cdot \frac{\ln (\bar{d})}{\frac{\sigma}{l}}\right)\right) \mathrm{d} \bar{d}
$$

For clarification purposes, $\mathrm{d} \bar{d}$ signifies that the integral is being taken with respect to the variable $\bar{d}$. Because the locations of terminals are independent the expected number of terminals within range of the base station $t$ in a cell of $M$ terminals is:

$$
t=M \cdot \int_{0}^{K} \frac{\bar{d}}{K^{2}}\left(1-\operatorname{erf}\left(\frac{10}{\sqrt{2} \ln (10)} \cdot \frac{\ln (\bar{d})}{\frac{\sigma}{l}}\right)\right) \mathrm{d} \bar{d}
$$

We would like to solve for $K$ but unfortunately there is no closed form solution for this equation. However this expression can be evaluated numerically. The results of our analysis match our simulations very closely as is shown in section 6.4.

\subsection{Simulation Results}

In order to quantify the benefits of the pilot power protocol (PPP), the results of our simulations as well as our analysis are presented in this section. Figure 6.8 shows the transmission range factor $K$ chosen using the pilot power protocol with respect to the 
number of mobile terminals $M$ in the cell using both simulation and analysis for two different values of $\gamma$. From Figure 6.8, we observe that as the number of terminals increases, the chosen $K$ increases (transmission range decreases) because there is a larger number of potential paths to the base station. Figure 6.8 also shows that the results of our analysis presented in section 6.3 match our simulations very closely.

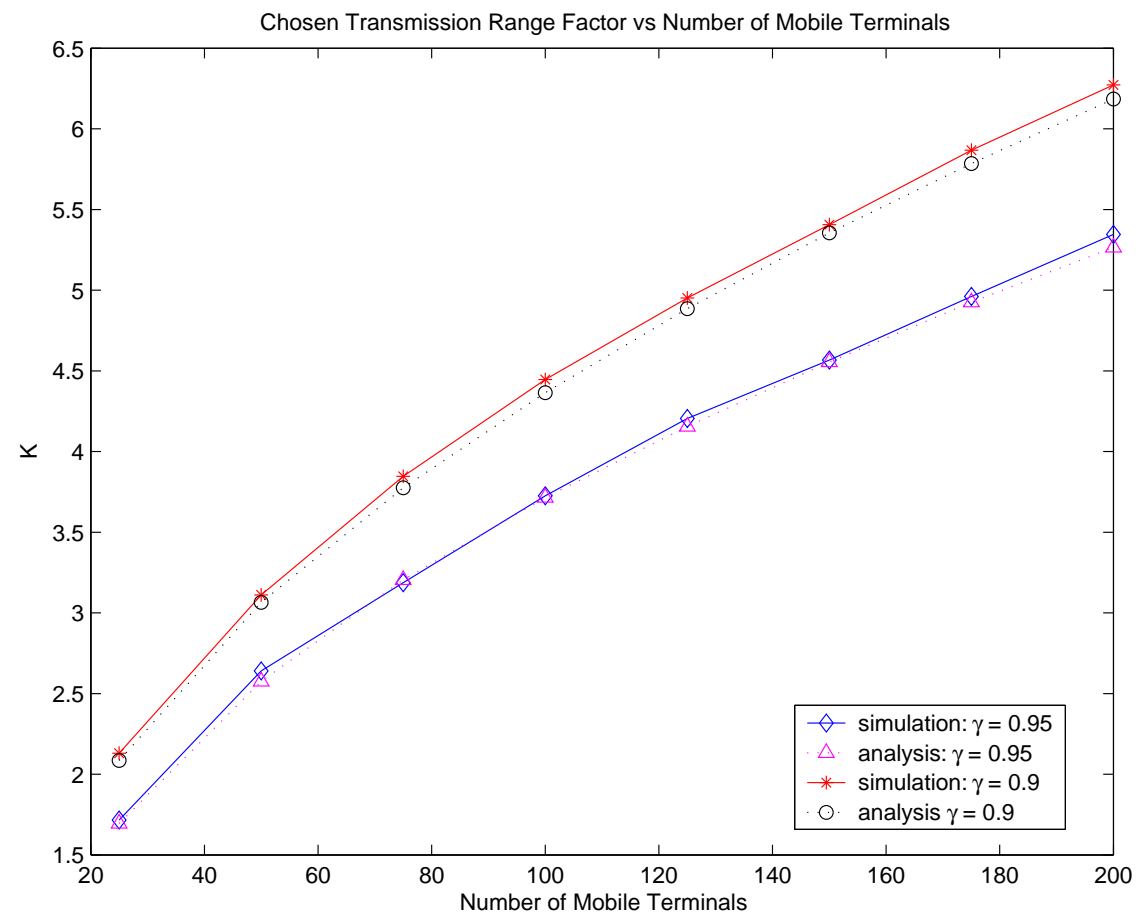

Figure 6.8: Chosen Transmission Range Factor vs Number of Mobile Terminals

Figure 6.9 shows our simulations of the percentage of mobile terminals that receive coverage with respect to the number of mobile terminals in the cell when choosing the transmission range factor using the pilot power protocol with $\gamma=0.9$ and $\gamma=0.95$ as well as when using a constant transmission range with $K=3$. This figure shows that when using the pilot power protocol, coverage remains roughly constant for a given $\gamma$ regardless of the number of terminals in the cell which would be unknown in a multihop cellular network. Furthermore, the pilot power protocol performs better than using a constant transmission range which is the method used in the majority of previous works in multihop cellular networks [68][12][73]. This is because the pilot power protocol adjusts 
the transmission range of terminals autonomously so that a sufficient required coverage level is achieved for all densities of mobile terminals whereas in a constant transmission range scheme terminals in sparsely populated networks will not receive adequate coverage.

Furthermore, Figure 6.9 shows coverage remains roughly constant for a given $\gamma$ and is not dependent on the number of terminals within the cell which would be unknown in a multihop cellular network. This is important and very impressive for a network that relies on multiple hops because the network will be able to autonomously adjust its pilot power to obtain optimal performance while guaranteeing to meet the coverage requirement regardless of the number of terminals within the cell.

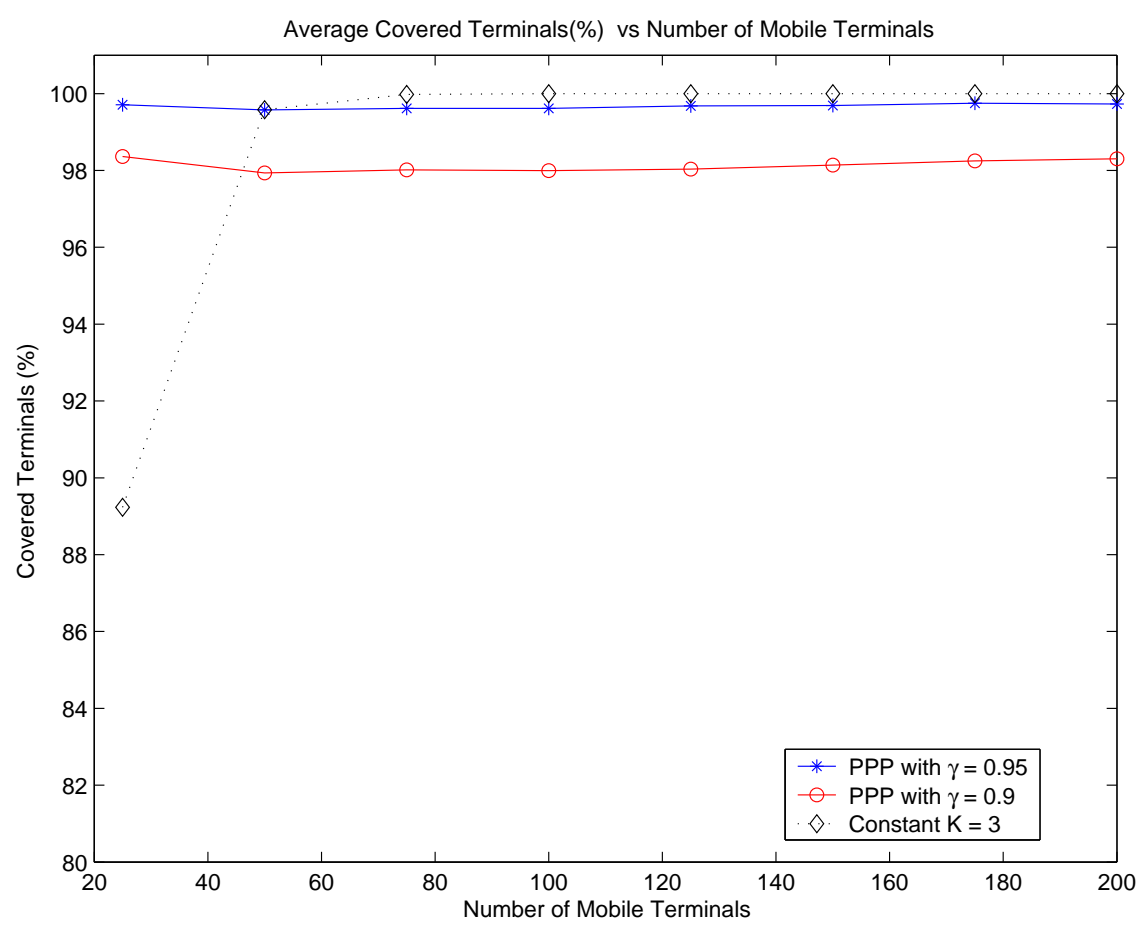

Figure 6.9: Percentage Covered Terminals vs Number of Mobile Terminals

Figure 6.10 shows our simulations of the average SINR at the base station with respect to the number of terminals in the cell when choosing the transmission range factor using the pilot power protocol with $\gamma=0.9$ and $\gamma=0.95$ as well as when using a constant transmission range with $K=3$. This figure shows that the SINR at the base station is higher when using the pilot power protocol than when using a constant transmission 
range. The only exception being sparsely populated cells which have slightly higher SINR due to holes in coverage that lead to less traffic being forwarded to the base station. The reason that the SINR at the base station is higher when using the pilot power protocol is that in densely populated cells the transmission range factor $K$ can be increased as shown in Figure 6.8 which leads to a reduction in interference and a higher SINR at the base station.

Furthermore, Figure 6.10 shows that the bottleneck that exists at the base station in a regular multihop cellular network does not occur in the autonomous infrastructure multihop cellular network with the pilot power protocol because mobile terminals can be added to the network without decreasing the SINR at the base station. This implies that growth of the network can occur organically with user connected access points and without the need for further infrastructure deployment by the network operator.

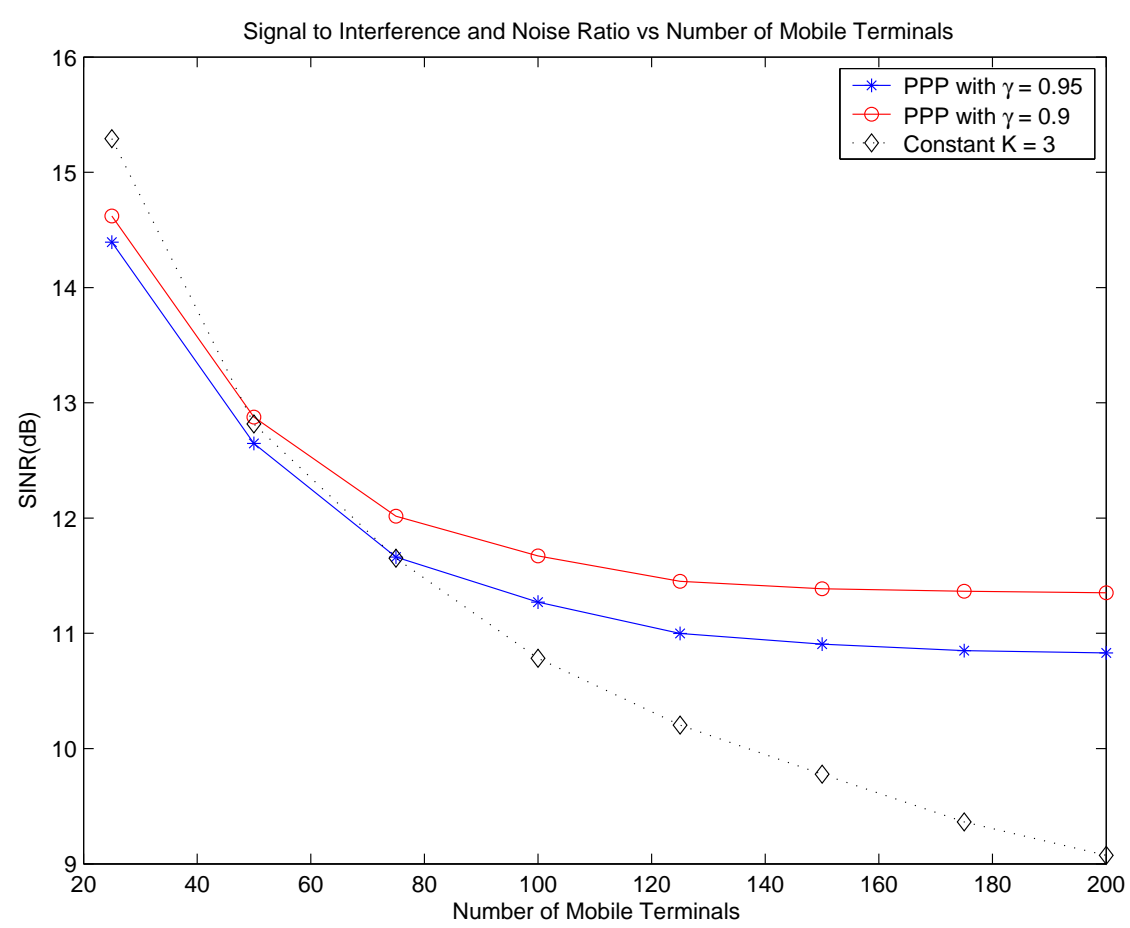

Figure 6.10: Average SINR vs Number of Mobile Terminals

Figure 6.11 shows our simulations of the power consumption per covered terminal with respect to the number of terminals in the cell when choosing the transmission range factor 
using the pilot power protocol with $\gamma=0.9$ and $\gamma=0.95$ as well as when using a constant transmission range with $K=3$. This figure shows that the average power consumption is lower when using the pilot power protocol than when using a constant transmission range. The only exception to this being sparsely populated cells which have lower power consumption but also suffer from holes in coverage. The reason that the overall power consumption is lower when using the pilot power protocol is that in densely populated cells the transmission range factor $K$ can be increased. Power consumption decreases as we increase $K$ because using smaller transmission ranges allows us to decrease the transmit power of each terminal. Although more transmissions are required for traffic to reach its destination, the reduction of path loss allows us to reduce the transmit power sufficiently so that the average power consumption per terminal is lower when using a larger $K$.

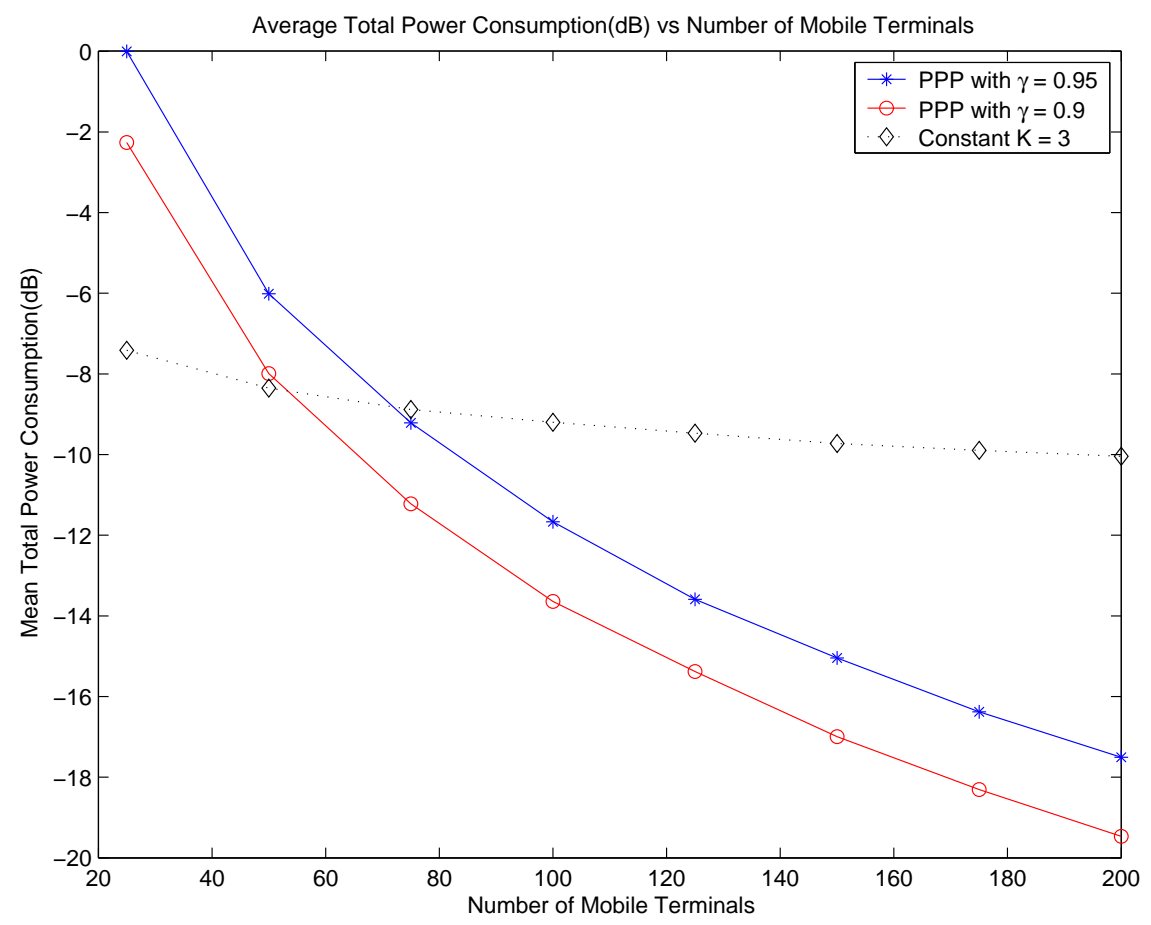

Figure 6.11: Average Power Consumption vs Number of Mobile Terminals

Figure 6.12 shows our simulations of the average number of hops required for traffic emanating from a mobile terminal to reach the base station or an access point with 
respect to the number of terminals in the cell when choosing the transmission range factor using the pilot power protocol with $\gamma=0.9$ and $\gamma=0.95$ as well as when using a constant transmission range with $K=3$. This figure shows that increasing the number of terminals in the cell will slightly increase the number of hops required for traffic to reach the backbone network when using the pilot power protocol. This is because the pilot power protocol decreases the transmission range of mobile terminals when there are many terminals in the cell. Despite the large reduction in transmission ranges of terminals in densely populated cells, the average number of hops remains below 1.83 and 2.16 when using the pilot power protocol with $\gamma=0.95$ and $\gamma=0.9$, respectively. Furthermore, the use of the pilot power protocol results in more consistent performance in terms of mean hop count than does the use of a constant transmission range $K=3$.

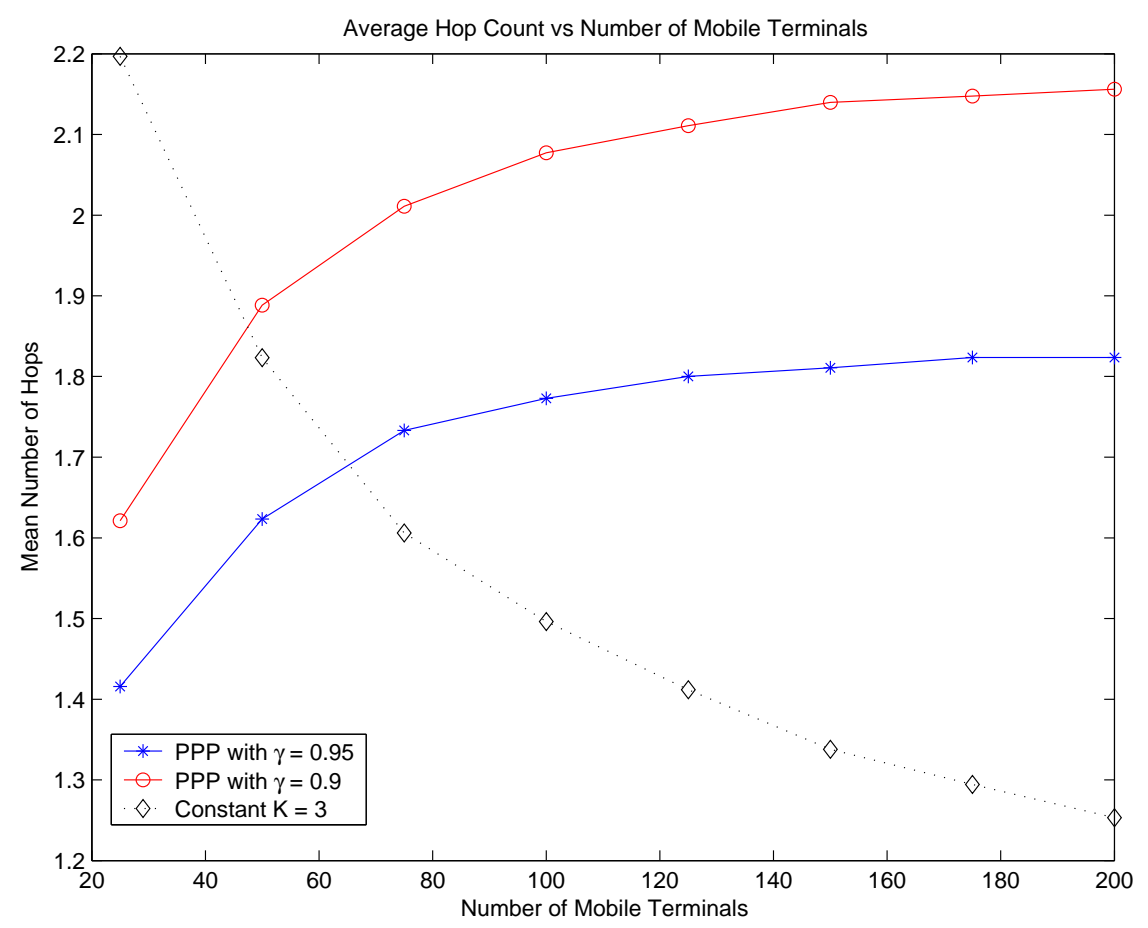

Figure 6.12: Average Hop Count vs Number of Mobile Terminals

From these figures we see that the pilot power protocol automatically and autonomously decreases $K$ (increases transmission range) when there happens to be less mobile terminals within the cell to meet the coverage requirement which is a function of $\gamma$. In cells 
that are densely populated, a higher value of $K$ is chosen by the protocol to improve performance in terms of SINR and power consumption while still obtaining the same required coverage. Increasing $K$ improves SINR and power consumption as was seen in Figures 6.2 and 6.3 .

Figure 6.13 shows our simulations of the percentage of mobile terminals that receive coverage with respect to the parameter $\gamma$ in a regular multihop cellular network with 200, 100 and 50 mobile terminals. From this graph we see further proof that coverage remains constant for a chosen $\gamma$ and is not dependent on the number of terminals within the cell. Using this graph, a suitable value of $\gamma$ can be found to meet a required coverage level $V$ specified by the network operator. In fact using a quantitative curve fitting tool, an equation that relates $V$ to $\gamma$ was found. This equation was also plotted on Figure 6.13 and we can see that it gives a good approximation of the results obtained via simulation. This equation used the following 7th degree polynomial to approximate the relationship between $V$ and $\gamma$ :

$$
\gamma=p_{1} \cdot x^{7}+p_{2} \cdot x^{6}+p_{3} \cdot x^{5}+p_{4} \cdot x^{4}+p_{5} \cdot x^{3}+p_{6} \cdot x^{2}+p_{7} \cdot x+p_{8}
$$

where $\mathrm{x}$ is a scaled version of the required coverage $V$ normalized by its mean 68.69 and its standard deviation 36.75

$$
x=\frac{(V-68.69)}{36.75}
$$

and the coefficients of equation 6.18 are found in Table 6.1 .

To meet the coverage requirement $V$ specified by the network operator, access points can use the pilot power protocol with $\gamma$ found using equations 6.18 and 6.19 . The pilot power protocol will find the transmission range which is optimal in terms of SINR and power consumption that meets the coverage requirement regardless of the number of terminals in the cell. 
Chapter 6. Pilot Power Protocol for Optimal Transmission Range 110

Table 6.1: Coefficients of Equation Relating $\gamma$ and $V$

\begin{tabular}{|c|c|}
\hline \hline Coefficient & Value \\
\hline p1 & 7.127 \\
p2 & 27.37 \\
p3 & 24.55 \\
p4 & -16.76 \\
p5 & -18.91 \\
p6 & 5.745 \\
p7 & 24.57 \\
p8 & 67.82 \\
\hline
\end{tabular}

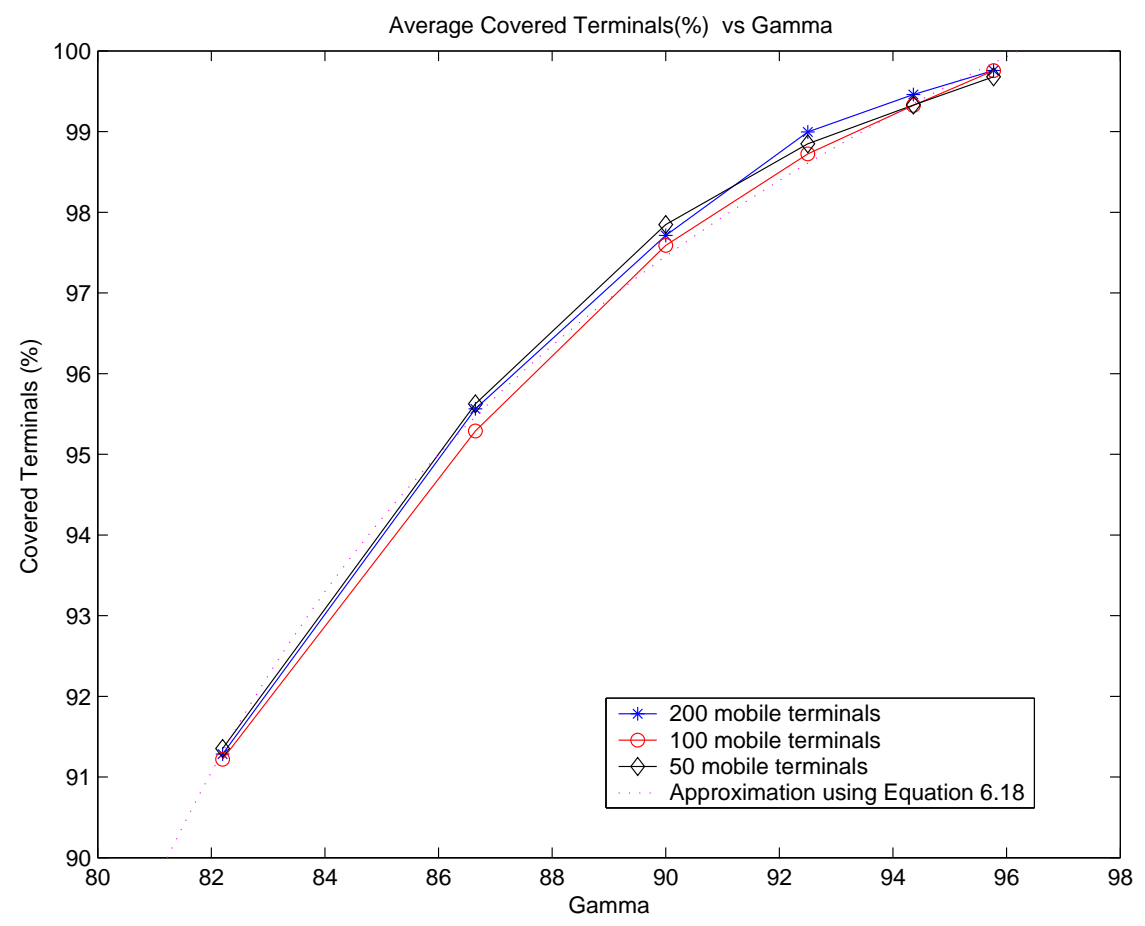

Figure 6.13: Percentage Covered Terminals vs Gamma 


\subsection{Optimality of Pilot Power Protocol}

The objective of the pilot power protocol was to find a transmission range factor $K$ that formed a multihop cellular network which guaranteed a minimum required percentage of covered terminals while optimizing SINR and power consumption. In other words, assume that $F$ is the set of transmission range factors with which a multihop cellular network meets or exceeds the minimum required coverage level. Our goal was to create a protocol that found the element of the set $F$ that produces the multihop cellular network with the highest SINR and lowest power consumption.

To prove that the pilot power protocol achieves our objectives, we provide the following analysis. For any network topology let us assume that:

$$
\text { coverage }_{K=i}<\text { coverage }_{K=j} \quad \forall i, j \text { where } i>j
$$

$$
\operatorname{SINR}_{K=i}>\operatorname{SINR}_{K=j} \quad \forall i, j \text { where } i>j
$$

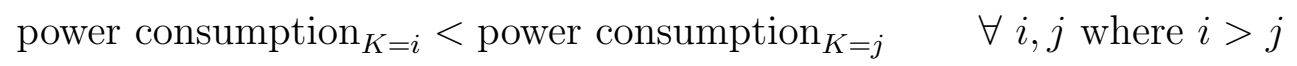

These are reasonable assumptions that are confirmed by the simulations of section 6.1.

We denote the minimum required percentage of covered terminals as $V$ and the transmission range factor that achieves this coverage as $a$. Therefore

$$
\text { coverage }_{K=a}=V
$$

The set of all transmission range factors $F$ that achieve coverage $\geq V$ are all transmission range factors $K \leq a$ according to equations 6.20 and 6.23 .

To achieve our objective it is required that the SINR be maximized and the power consumption be minimized given the constraint $K \leq a$. To optimize SINR and power 
consumption given this constraint we must choose $K=a$ due to equations 6.21 and 6.22 . This is because according to equations 6.21 and 6.22, performance in terms of SINR and power consumption deteriorates as we decrease $K$. This implies that for the pilot power protocol to work optimally it must determine the transmission range factor that achieves coverage $=V$.

Because the results of our analysis show that optimal SINR and power consumption levels occur exactly at the required coverage levels, the pilot power protocol will achieve our objectives. The pilot power protocol achieves this by determining the transmission range factor $K$ that achieves a constant coverage level $V$ regardless of the density of terminals in the cell. This was shown to be true in Figure 6.9. More specifically, by using Figure 6.13 or equation 6.18 , the value of $\gamma$ can be found from the required coverage level $V$ specified by the network operator. Using $\gamma$ the pilot power protocol can determine the transmission range factor $K$ that achieves the exact required coverage level $V$ for any given density of randomly distributed mobile terminals. Because the transmission range factor is set such that the coverage level is exactly at the required level, performance in terms of SINR and power consumption is optimal.

\subsection{Chapter Summary}

In this chapter, we proposed a pilot power protocol for the autonomous infrastructure multihop cellular network. Although this algorithm was designed for use by access points, it can equally be used to find optimal transmission ranges for base stations in both regular and autonomous infrastructure multihop cellular networks. The results of our simulations and analysis showed that by selecting a suitable $\gamma$, a required percentage of covered terminals is obtained. Furthermore, the pilot power protocol determines the pilot power level which is optimal in terms of SINR and power consumption that meets the coverage requirement. This can be achieved regardless of the density of terminals in 
Chapter 6. Pilot Power Protocol for Optimal Transmission Range 113

a cell which is impressive for a network that relies on multiple hops.

We also showed that the bottleneck that exists at the base station in a regular multihop cellular network does not occur in the autonomous infrastructure multihop cellular network with the pilot power protocol because mobile terminals can be added to the network without decreasing the SINR at the base station as seen in Figure 6.10. This implies that growth of the network can occur organically with user connected access points and without the need for further costly infrastructure deployment by the network operator. 


\section{Chapter 7}

\section{Conclusion}

In this chapter, we present a summary of the work that has been presented in this thesis. After incorporating the work of the various chapters, several concluding remarks will be made. While the results in this thesis showed that the autonomous infrastructure multihop cellular network is a viable alternative for $4 \mathrm{G}$ networks, there are still some related issues that must be studied further. Ideas for future work will be presented in this chapter.

\subsection{Summary}

In recent years, research has been performed in the area of multihop cellular networks due to their potential for lower energy consumption, higher capacity and improved coverage [15]. In a multihop cellular network, the physical layer of mobile terminals is modified so that in addition to being able to transmit to base stations, mobile terminals are able to transmit directly to other mobile terminals. This allows mobile terminals to lower their maximum transmission range and use other terminals as relays to forward their traffic towards the base station.

In chapter 3 of this thesis, a complete system model of the multihop cellular network was presented. This includes the network model, the propagation model, the communi- 
cation channels and spreading codes, the minimum hop routing algorithm used to form the multihop cellular network, the time-division duplexing CDMA mechanism and the power control mechanism.

In chapter 4, we investigated the effects of intercell interference and correlated lognormal shadowing on system performance because real world networks consist of multiple cells and shadowing coefficients are found to be correlated [20]. We compare our results to prior works on the multihop cellular network which had only considered one-cell systems where shadowing coefficients of different mobile terminals were modeled as being independent. The capacity of a CDMA cellular network depends on the total interference at the base station which is comprised of both in-cell and intercell interference. In a traditional cellular network, the in-cell and intercell interference typically account for two-thirds and one-third of the total interference in a cell, respectively. Our simulation results show that the intercell interference in a multihop cellular network is decreased by as much as $28.7 \mathrm{~dB}$ as compared to a traditional cellular network. This means that the intercell interference which accounts for one-third of the total interference in a traditional cellular network is effectively eliminated in a multihop cellular network. Simulation results presented in this thesis show that in a network modeled with correlated lognormal shadowing consisting of multiple cells, the average SINR at the base station is larger than that of a traditional cellular network by $1.6 \mathrm{~dB}$ as opposed to being smaller by $0.3 \mathrm{~dB}$ in a network consisting of one cell where correlated lognormal shadowing is not considered. Simulation results also show that in a network consisting of many cells where lognormal shadowing coefficients are modeled as being correlated, the overall transmission power consumption is lower than in a traditional cellular network by $23 \mathrm{~dB}$ as opposed to 18 $\mathrm{dB}$ in a one-cell system with independent lognormal shadowing coefficients.

In a multihop cellular network, the base station and the mobile terminals surrounding it are responsible for handling a large amount of traffic for other terminals. This is because all traffic either emanates or is destined to the base station causing a large congestion of 
wireless traffic and interference near the base station making it the capacity bottleneck of the network. Typically, when the congestion near the base station becomes large, a new base station would be deployed to help handle some of the traffic. However, the construction cost of additional base stations is very high. In this thesis, we presented an alternate solution that is cost-effective, flexible and scalable to help alleviate the amount of traffic that base stations are required to handle.

In chapter 5 of this thesis, we proposed a novel multihop cellular network architecture where users can connect their mobile terminals to the backbone network to give them the functionality of an access point so that they can forward the traffic of other terminals directly onto the backbone network. In this architecture, we assume that a subset of the mobile terminals in the network are built with an additional interface giving them the potential to transmit directly onto the backbone network. In the future, the cost of manufacturing these mobile terminals may become sufficiently inexpensive that a large percentage of terminals could have this capability. Users can connect their mobile terminal to the backbone network using this interface to improve capacity, coverage and data rates in a certain area. We refer to a mobile terminal as an access point when the terminal is using this interface to establish a direct connection to the backbone network and is able to handle traffic for other users, as would a base station. Access points can connect to the backbone network using a broadband wireless access network, a LAN, a DSL connection, a CATV network or a PLC connection. Depending on their locations, other terminals are able to forward their traffic to a base station or to any access point that sends the traffic directly onto the backbone network. Therefore, the load on base stations is lower and further links to the backbone network are provided autonomously by the user. Allowing mobile terminals in a multihop cellular network to act as access points does not require any major modifications to the physical layer of mobile terminals because terminals already have the capability to transmit directly to one another. For this reason, the multihop cellular network architecture lends itself perfectly to having 
certain mobile terminals act as access points. This is not the case in traditional cellular networks because mobile terminals only have the capability to transmit to base stations. In this thesis we also present a mechanism to integrate access points into the minimum hop routing algorithm and a mechanism to integrate access points into the TDD-CDMA transmission in timeslot scheme. Results of simulations and analysis presented in this thesis show that our proposed solution leads to an increase in SINR at the base station, an increase in coverage and a decrease in overall power consumption as compared to a regular multihop cellular network.

An important aspect of the proposed architecture is the automatic configuration of the transmission parameters of access points. In a traditional cellular network, RF specialists monitor base station transmissions due to regulatory requirements and interference and coverage considerations. However, RF specialists are not able to adjust access point parameters and access points will have to determine their optimal transmission parameters autonomously. An access point has a limited capability depending on the mobile terminal's battery power and the bandwidth of its connection to the backbone network and is therefore only able to handle traffic for a limited number of terminals that are a certain number of hops away. An access point also has hardware limitations due to its power amplifier and its processing capability that restrict its maximum transmission power level. The access point will have to autonomously choose an optimal maximum transmission range that is high enough to ensure coverage of a sufficient number of terminals but not excessively high that it is causing too much interference. Clearly, the chosen maximum transmission range must involve the use of a transmission power level that is below the maximum transmission power level dictated by the hardware limitations. In our architecture access points and the terminals they are serving have the same transmission range to ensure that bidirectional communication is possible. Having an optimally chosen transmission range is essential because it has a crucial effect on the transmission patterns, interference levels, power consumption and coverage levels in a 
multihop cellular network.

An objective of this research was to develop a protocol that autonomously determines the optimal transmission range of access points and terminals for a given coverage requirement. In our architecture, an access point is restricted from transmitting data with a transmission power that is higher than its pilot power level. This implies that the transmission range of the access point is defined by its pilot power level. This is because only terminals that receive and can decode an access point's pilot signal transmit directly to that access point and are said to be within transmission range. This implies that we can meet our objective of optimizing the transmission range of mobile terminals by adjusting the pilot power levels of access points to optimal levels.

In chapter 6 of this thesis, we developed and presented a pilot power protocol for the autonomous infrastructure multihop cellular network which is used to determine optimal pilot power levels for both access points and base stations based on the density of mobile terminals within a cell. The pilot power level of an access point is directly related to its transmission range and the pilot power protocol is used to determine its optimal transmission range. The algorithm addressed the tradeoff that exists between coverage vs SINR and power consumption by autonomously increasing the transmission range in sparsely populated cells to achieve the required coverage level and autonomously decreasing the pilot power level in densely populated cells to improve SINR and power consumption while maintaining the required coverage level. Simulation results using the pilot power protocol show that the chosen transmission range decreases as we increase the number of terminals within the network. Results of mathematical analysis presented in this thesis confirm and match our simulation results very closely. We also show that the bottleneck that exists at the base station in a regular multihop cellular network does not occur in the autonomous infrastructure multihop cellular network with the pilot power protocol because mobile terminals can be added to the network without decreasing the SINR at the base station. Furthermore simulation results of an autonomous infrastruc- 
ture multihop cellular network with the pilot power protocol show that the percentage of terminals that receive coverage remains roughly constant, independent of the density of mobile terminals. This feature is crucial for any multihop network as the coverage of a terminal is dependent not only on its distance from a base station but also on the number and location of mobile terminals surrounding it. Therefore by adjusting a parameter within the pilot power protocol, a required percentage of covered terminals can be specified without prior knowledge of the location or number of terminals within a cell which would be unknown in an autonomous infrastructure multihop cellular network. We also show analytically that the pilot power protocol achieves optimal values of SINR and power consumption for a given coverage requirement.

\subsection{Concluding Remarks}

In this thesis we demonstrated that the autonomous infrastructure multihop cellular network is a viable option to be used in $4 \mathrm{G}$ and beyond networks. Our results showed that the SINR in a multihop cellular network is higher and the power consumption is lower than in a traditional cellular network. These results are due to the near elimination of intercell interference at the base station. Eliminating intercell interference will have a substantial effect on how spectrum can be allocated for neighboring cells. In a traditional cellular network frequency reuse cluster sizes can range from 1 to 7 or even higher. By eliminating intercell interference at the base station the same spectrum can be used in neighboring cells, i.e. a frequency reuse cluster size of 1 , without the reduction in capacity caused by interference from other cells reaching the base station that is common in traditional cellular networks.

We also proposed a novel network architecture called the autonomous infrastructure multihop cellular network that was found to increase SINR, lower power consumption and improve coverage as compared to a regular multihop cellular network. In this architecture 
users can connect their mobile terminals to the backbone network to handle traffic from other users and send it directly onto the backbone network as would a base station. We also proposed a pilot power protocol that can be used by access points and base stations to autonomously determine their optimal pilot power levels and transmission ranges. When using this protocol, the network operator is only required to specify the required coverage level and the protocol will autonomously set the pilot power and transmission ranges of terminals so that optimal values of SINR and power consumption are obtained. This can be achieved regardless of the number of terminals in a cell which is impressive for a network that relies on multiple hops.

We showed that the bottleneck that exists at the base station in a regular multihop cellular network is effectively eliminated in the autonomous infrastructure multihop cellular network using the pilot power protocol. Furthermore the scalability of the autonomous infrastructure multihop cellular network is better than the multihop cellular network because network growth can occur in an organic manner. This stems from our results that show that by increasing the number of access points, the capacity of the network will increase. If the capacity of the network is increased, a larger number of users can be supported. With a larger number of mobile terminals, we can envision that more of these terminals will have links to the backbone network and will act as access points. This will lead to a further increase in capacity. Following this reasoning, we can see how this flexible network is in fact scalable and how growth of the network can occur in an organic way. This architecture is advantageous to the network operator because it will not need to invest in the further deployment of expensive infrastructure. Moreover, the network operator may be provided with more traffic, leading to more revenue due to the network's higher capacity. The network operator is only responsible for managing the network domain controller which is in charge of call establishment and issues involving coordination, security and billing.

In this thesis, the line between mobile terminals and base stations is blurred because 
mobile terminals are allowed to act like base stations and handle traffic for other users by sending it directly onto the backbone network. A mobile terminal performing the functionality of an access point can be seen as a 'plug-and-play base station' if its connection to the backbone is hardwired or as a 'wireless base station' if its connection to the backbone network is through a wireless access network. One can even envision a multihop cellular network without a base station where access points are solely responsible for handling the traffic of mobile terminals as discussed in the Autonomous Mode of Operation in section 5.7. This proposed networking concept is revolutionary in the sense that it puts the control of infrastructure deployment partly in the hands of the user. This could have the effect of stimulating the deployment of future wireless services.

\subsection{Future Research}

While the results in this thesis showed the many advantages of the autonomous infrastructure multihop cellular network, there are still some related issues that must be studied further. Firstly, the effects of mobility of terminals should be investigated because mobility of terminals is a reality in an actual network and this would likely have an effect on our results. Secondly, to stimulate cooperation, mobile terminals that act as access points should be rewarded for taking on the burden of handling traffic for other terminals. Ideas for an incentive-based scheme were outlined in this thesis but further work is required for a reward scheme to be devised. Thirdly, in our simulations all mobile terminals have an equal chance of being connected to the backbone network and performing the functionality of an access point. However, in a real network a mobile terminal that is close to an access point may have a greater chance of itself being connected to the backbone network. Therefore, in future research our simulations will incorporate a correlated location-dependent model to determine which terminals become access points. Fourthly, the autonomous infrastructure multihop cellular network and pilot power protocol were 
modeled using CDMA for multiple access. However, this architecture and protocol were intentionally designed so that they would be applicable to other forms of multiple access that are robust to interference like OFDMA, which is being used in 3GPP LTE and WiMAX. In future research the work presented in this thesis will be adapted for use in OFDMA networks. Finally, mobile terminals may not be randomly distributed in a real network and may be clustered in certain areas. This may have an effect on the performance of the pilot power protocol. Therefore the performance of the pilot power protocol should be investigated for networks where terminals are not randomly distributed. 


\section{Bibliography}

[1] Third Generation Partnership Project (3GPP). Opportunity driven multiple access (ODMA). Tech. Rep. 25.924, December 1999.

[2] M. Asawa and W. Stark. Optimal scheduling of handoffs in cellular networks. IEEE/ACM Transactions on Networking, Vol. 3, No. 4, pages 428-441, June 1996.

[3] K.G. Beauchamp. Walsh Functions and their Applications. London: Academic Press, 1975.

[4] T. Beniero, S. Redana, J. Hamalainen, and B. Raaf. Effect of relaying on coverage in 3GPP LTE-Advanced. IEEE Vehicular Technology Conference VTC, 2009.

[5] J. Bingham. ADSL, VDSL, and Multicarrier Modulation. John Wiley, December 1999.

[6] A. Bletsas and A. Lippman. Implementing cooperative diversity antenna arrays with commodity hardware. IEEE Communications Magazine, Vol. 44, No. 12, pages 3340, December 2006.

[7] N. Chandran and M. Valenti. Three generations of cellular wireless systems. IEEE Potentials, Vol. 20, No. 1, pages 32-35, 2001.

[8] J. Cho and Z. Haas. On the throughput enhancement of the downstream channel in cellular radio networks through multihop relaying. IEEE Journal on Selected Areas in Communications, Vol. 22, No. 7, pages 1206-1219, September 2004. 
[9] J. Chung, H. Cho, K. Jin, M. Cho, and H. Kim. A cooperative MIMO mobile multihop relay for cellular networks. Proceedings of WSEAS Conference on Electronics, Hardware, Wireless and Optical Communications, pages 116-120, February 2007.

[10] M. DeFaria. Routing in a multihop cellular network. Master's thesis, University of Toronto, 2004.

[11] M. DeFaria and E.S. Sousa. A minimum hop routing algorithm for multihop cellular networks. Journal of Communications and Information Systems co-sponsored by the IEEE Communications Society and the Brazilian Telecommunications Society, Special Issue on Sensors and Ad Hoc Networks, Vol. 19, No. 3, pages 27-39, 2004.

[12] M. DeFaria and E.S. Sousa. Autonomous infrastructure based multihop cellular networks. IEEE Personal Indoor Mobile Radio Communications Conference (PIMRC), 2009.

[13] M. DeFaria and E.S. Sousa. An optimal transmission range protocol for autonomous infrastructure based multihop cellular networks. IEEE Transactions on Wireless Communications, In Submission, 2009.

[14] M. DeFaria and E.S. Sousa. Pilot power protocol for autonomous infrastructure based multihop cellular networks. IEEE Global Communications Conference (GLOBECOM), 2009.

[15] M. DeFaria and E.S. Sousa. Effect of intercell interference on the SNIR of a multihop cellular network. IEEE Vehicular Technology Conference (VTC), pages 3107-3111, June 2005.

[16] X. Fang, C. Zhu, and P. Fan. Greedy-based dynamic channel assignment strategy for cellular mobile networks. IEEE Communications Letters, vol 4, no. 7, pages 215-217, July 2000. 
[17] Jacobus Glas. Non-cellular Wireless Communication Systems. PhD thesis, Technische Universiteit Delft, 1996.

[18] S.W. Golomb. Shift register sequences. Holden-Day Inc., 1967.

[19] G. Golub and C. Van Loan. Matrix Computations. John Hopkins University Press, 1996.

[20] V. Graziano. Propagation correlations at $900 \mathrm{MHz}$. IEEE Transactions on Vehicular Technology, pages 182-189, November 1978.

[21] L. Guan, J. Zhang, J. Li, G. Liu, and P. Zhang. Spectral efficient frequency allocation scheme in multihop cellular networks. Proceedings of IEEE Vehicular Technology Conference Fall, pages 1446-1450, 2007.

[22] R. Hekmat and P. Van Mieghem. Degree distribution and hopcount in wireless adhoc networks. IEEE International Conference On Networks (ICON), pages 603-609, October 2003.

[23] H. Holma and A. Toskala. WCDMA for UMTS, 4th edition. Hoboken:Wiley, 2007.

[24] T. Hou and T. Tsai. On the cluster based dynamic channel assignment for multihop ad hoc networks. Journal of Communications and Networks, Vol. 4, No. 1, March 2002 .

[25] H. Hu, H. Yanikomeroglu, D. Falconer, and S. Periyalwar. Range extension without capacity penalty in cellular networks with digital fixed relays. IEEE Global Communications Conference GLOBECOM, 2004.

[26] D. Johnson J. Broch and D. Maltz. The dynamic source routing protocol for mobile ad hoc networks. IETF Draft, November 2000. 
[27] K. Jacobson and W. Krzymien. Cooperative vs multihop relaying in cellular systems. IEEE Personal Indoor Mobile Radio Communications Symposium PIMRC, September 2009.

[28] B.M. Jakobsson, J. Hubaux, and L. Buttyan. Micro-payment scheme encouraging collaboration in multi-hop cellular networks, November 2004.

[29] S. Jeon and S. Lee. A relay-assisted handover technique with network coding over multihop cellular networks. IEEE Communications Letters, Vol. 11, No. 3, pages 252-254, March 2007.

[30] K. Johansson, A. Furuskar, P. Karlsson, and J. Zander. Relation between base station characteristics and cost structure in cellular systems. IEEE PIMRC, pages 2627-2631, 2004.

[31] T. Klingenbrunn and P. Mogensen. Modelling cross-correlated shadowing in network simulations. IEEE Vehicular Technology Conference(VTC), pages 1407-1411, 1999.

[32] E. Kudoh and F. Adachi. Power and frequency efficient wireless multi-hop virtual cellular concept. Transactions on Communications (4), pages 1613-1621, 2005.

[33] A. Kusuma and L. Andrew. Minimum power routing for multihop cellular networks. IEEE GLOBECOM, 2002.

[34] M. Kwok and H. Wang. Adjacent cell interference analysis of the reverse link in CDMA cellular radio systems. IEEE Personal, Indoor and Mobile Radio Communications PIMRC, pages 446-450, September 1995.

[35] E. Kwon, J. Lee, M. Do, and K. Jung. Comparison of symmetric and asymmetric routing for fixed two-hop cellular relaying network. IEEE Communication Letters, Vol. 11, No. 5, pages 378-380, May 2007. 
[36] L. Le and E. Hossain. Multihop cellular networks: Potential gains, research challenges and a resource allocation framework. IEEE Communications Magazine, pages 66-73, September 2007.

[37] X. Li, B. Seet, and P. Chong. Multihop cellular networks: Technology and economics. Computer Network, Vol. 52, No. 9, pages 1825-1837, June 2008.

[38] Y. Lin and Y. Hsu. Multihop cellular: A new architecture for wireless communications. IEEE Infocom, 2000.

[39] M. Lott, M. Weckerle, W. Zirwas, H. Li, and E. Schulz. Hierarchical cellular multihop networks. Proceedings of the 5th European Personal Mobile Communications Conference, pages 37-43, September 2003.

[40] H. Luo, R. Ramjee, P. Sinha, L. Li, and S. Lu. UCAN: A unified cellular and ad-hoc network architecture. Proceedings of ACM MOBICOM, page 353367, 2003.

[41] N. Marathe, U. Desai, and S. Merchant. Base station selection strategy in multihop cellular networks: A new approach. Proceedings of the International Conference on Signal Processing, Communications and Networking, January 2008.

[42] D. Martn-Sacristn, J. Monserrat, J. Cabrejas-Peuelas, D. Calabuig, S. Garrigas, and N. Cardona. On the way towards fourth-generation mobile: 3GPP LTE and LTE-Advanced. EURASIP Journal on Wireless Communications and Networking, ID:354089, 2009.

[43] A. Nosratinia, T. Hunter, and A. Hedayat. Cooperative communication in wireless networks. IEEE Communications Magazine, Vol. 42, No. 10, pages 74-80, October 2004.

[44] Institute of Electrical and Electronics Engineers. IEEE standard for local and metropolitan area networks - part 16: Air interface for fixed and mobile broad- 
band wireless access systems - amendment for physical and medium access control layers for combined fixed and mobile operation in licensed bands. IEEE Standard 802.16e, 2005 .

[45] Institute of Electrical and Electronics Engineers. Amendment to IEEE standard for local and metropolitan area networks - part 16: Air interface for broadband wireless access systems - multihop relay specification. IEEE Standard 802.16j, June 2009.

[46] R. Pabst, B. Walke, D. Schultz, P. Herhold, H. Yanikomeroglu, S. Mukherjee, H. Viswanathan, M. Lott, W. Zirwas, M. Dohler, H. Aghvami, D. Falconer, and G. Fettweis. Relay-based deployment concepts for wireless and mobile broadband radio. IEEE Communications Magazine, pages 80-89, September 2004.

[47] N. Pavlidou, A. Vinck, J. Yazdani, and B. Honary. Power line communications: State of the art and future trends. IEEE Communications Magazine, Vol. 41, pages 34-40, April 2003.

[48] K.M. Pepe and B.R. Vojcic. Cellular multihop networks and the impact of routing on the SNIR and total power consumption. Proceedings of MMT'02, June 2002.

[49] M.R. Perlman and Z.J. Haas. On the impact of alternate path routing for load balancing in mobile ad hoc networks. Proceedings of IEEE/ACM MobiHoc 2000, August 2000 .

[50] S. Peters, A. Panah, K. Truong, and R. Heath. Relay architectures for 3GPP LTE-Advanced. EURASIP Journal on Wireless Communications and Networking, ID:618787, 2009.

[51] R. Prasad, W. Mohr, and W. Konhauser. Third Generation Mobile Communication Systems. Boston: Artech House, 2000.

[52] J. Proakis and M. Salehi. Digital Communications. New York: McGraw-Hill, 2008. 
[53] Third Generation Partnership Project. 3GPP long term evolution: Technical specifications and technical reports for a UTRAN-based 3GPP system, release 8. Tech. Rep. 21.101, January 2009.

[54] Third Generation Partnership Project. Long term evolution - advanced: Requirements for further advancements for E-UTRA, v.8.0.1. Tech. Rep. 36.913, March 2009 .

[55] R. Rabbat and K. Siu. QoS support for integrated services over CATV. IEEE Communications Magazine, Vol. 37, No. 1, January 1999.

[56] A. Radan. Quantitative Analysis of Multihop CDMA Cellular Networks. PhD thesis, Queen's University, 2009.

[57] A. Radan and H. Hassanein. Capacity enhancement in CDMA cellular networks using multi-hop communication. IEEE Symposium on Computers and Communications, pages 832-837, 2006.

[58] R. Ramanathan and J. Redi. A brief overview of ad hoc networks: Challenges and direction. IEEE Communications Magazine, pages 20-22, May 2002.

[59] H.F.A. Roefs. Binary Sequences for Spread-Spectrum Multiple-Access Communications. PhD thesis, University of Illinois, 1977.

[60] A. Safwat. A-cell: A novel multi-hop architecture for $4 \mathrm{G}$ and $4 \mathrm{G}+$ wireless networks. Proceedings of IEEE VTC03 Fall, pages 2931-2935, 2003.

[61] N. Salem, L. Buttyan, J. Hubaux, and B.M. Jakobsson. A charging and rewarding scheme for packet forwarding in multi-hop cellular networks. Proceedings of the 4th ACM international symposium on Mobile ad hoc networking and computing (MobiHoc), pages 13-24, 2003. 
[62] D.V. Sarwate and M.B. Pursley. Crosscorrelation properties of pseudorandom and related sequences. Proceedings of the IEEE Vol. 68, pages 593-619, May 1980.

[63] A. Sendonaris, E. Erkip, and B. Aazhang. User cooperation diversity - part I: System description - part II: Implementation aspects and performance analysis. IEEE Transactions on Communications, Vol. 51, No. 11, pages 1927-1948, November 2003.

[64] I. Siomina and D. Yuan. Minimum pilot power for service coverage in WCDMA networks. Wireless Networks, Vol. 14, No. 3, pages 393-402, 2008.

[65] T.B. Sorensen. Slow fading cross-correlation versus azimuth separation of base stations. Electronic Letters, pages 127-129, January 1999.

[66] E.S. Sousa. Autonomous infrastructure wireless networks. IST Mobile and Wireless Communications Summit, 2007.

[67] E.S. Sousa and J.A. Silvester. Spreading code protocols for distributed spreadspectrum packet radio networks. IEEE Transactions on Communications, vol. 36, pages 272-281, 1988.

[68] Y. Tam, R. Benkoczi, H. Hassanein, and S. Akl. Optimal channel assignment in multi-hop cellular networks. IEEE GLOBECOM, pages 731-735, 2007.

[69] International Telecommunications Union. About mobile technology and IMT-2000. Technical report, ITU, 2005.

[70] International Telecommunications Union. Worldwide mobile cellular subscribers to reach 4 billion mark late 2008. Technical report, ITU, September 2008.

[71] A.J. Viterbi, A.M. Viterbi, K. Gilhousen, and E. Zehavi. Soft handoff extends CDMA cell coverage and increases reverse link capacity. Mobile Communications Advanced Systems and Components, pages 541-551, 1994. 
[72] A.J. Viterbi and E. Zehavi. Other-cell interference in cellular power controlled CDMA. IEEE Trans. Comm., vol. 42, pages 1501-1504, 1994.

[73] H. Wei and R. Gitlin. Two hop relay architecture for next generation WWAN/WLAN integration. IEEE Wireless Communications, pages 24-30, April 2004 .

[74] H. Wu, C. Qiao, S. De, and O. Tonguz. Integrated cellular and ad hoc relaying systems: iCAR. IEEE Journal on Selected Areas in Communications 19 (10), page $21052115,2001$.

[75] K. Wu and J. Harms. Multipath routing for mobile ad hoc networks. IEEE Communication Society, Special Issue on Innovations in Ad Hoc Mobile Pervasive Networks, March 2002.

[76] X. Wu, B S. Chan, Mukherjee, and B. Bhargava. MADF: Mobile-assisted data forwarding for wireless data networks. Journal of Communications and Networks 6 (3), pages 216-225, 2004.

[77] S. Yang. 3G CDMA2000 Wireless System Engineering. Artech House Publishers, 2004 .

[78] H. Yanikomeroglu. Cellular multihop communications: Infrastructure-based relay network architecture for 4G wireless systems. Proceedings of 22nd Queen's Biennial Symposium on Communications, 2004.

[79] A. Zadeh and B. Jabbari. Analysis and modeling of upstream throughput in multihop packet CDMA cellular networks. IEEE Transactions on Communications, Vol. 54, No. 4, pages 680-692, April 2006. 
[80] A.N. Zadeh, B. Jabbari, R. Pickholtz, and B. Vojcic. Self-organizing packet radio ad hoc networks with overlay (SOPRANO). IEEE Commun. Mag. vol. 40, pages 149-157, June 2002.

[81] J.J. Zhou and Y.R. Yang. PARCelS: Pervasive ad-hoc relaying for cellular systems. Proceedings of 1st Mediterranean Workshop on Ad-Hoc Networks, page 16, 2002.

[82] H. Zhu, T. Buot, R. Nagaike, and S. Harman. Load balancing in WCDMA systems by adjusting pilot power. Proceedings of 5th International Symposium on Wireless Personal Multimedia Communications, pages 936-940, 2002. 